University of Louisville

ThinkIR: The University of Louisville's Institutional Repository

8-2017

\title{
Spiritual transcendence as a sixth personality factor in Russia : cross-cultural generalizability and influence on psychosocial outcomes.
}

Ksenia Kopeikin

University of Louisville

Follow this and additional works at: https://ir.library.louisville.edu/etd

Part of the Personality and Social Contexts Commons

\section{Recommended Citation}

Kopeikin, Ksenia, "Spiritual transcendence as a sixth personality factor in Russia : cross-cultural generalizability and influence on psychosocial outcomes." (2017). Electronic Theses and Dissertations. Paper 2741.

https://doi.org/10.18297/etd/2741

This Doctoral Dissertation is brought to you for free and open access by ThinkIR: The University of Louisville's Institutional Repository. It has been accepted for inclusion in Electronic Theses and Dissertations by an authorized administrator of ThinkIR: The University of Louisville's Institutional Repository. This title appears here courtesy of the author, who has retained all other copyrights. For more information, please contact thinkir@louisville.edu. 


\title{
SPIRITUAL TRANSCENDENCE AS A SIXTH PERSONALITY FACTOR IN RUSSIA: CROSS-CULTURAL GENERALIZABILITY AND INFLUENCE ON PSYCHOSOCIAL OUTCOMES
}

\author{
By \\ Ksenia Kopeikin \\ B.A., University of Missouri-Columbia, 2009 \\ M.Ed., University of Oklahoma, 2011
}

\begin{abstract}
A Dissertation
Submitted to the Faculty of the

College of Education and Human Development of the University of Louisville in Partial Fulfillment of the Requirements for the Degree of

Doctor of Philosophy in Counseling and Personnel Services

Department of Educational and Counseling Psychology, Counseling, and College Student Personnel

University of Louisville

Louisville, Kentucky
\end{abstract}

August 2017 

SPIRITUAL TRANSCENDENCE AS A SIXTH PERSONALITY FACTOR IN RUSSIA: CROSS-CULTURAL GENERALIZABILITY AND INFLUENCE ON PSYCHOSOCIAL OUTCOMES

By

Ksenia Kopeikin

B.A., University of Missouri-Columbia, 2009

M.Ed., University of Oklahoma, 2011

A Dissertation Approved on

July 20, 2017

By the Following Dissertation Committee:

Mark Leach, Ph.D.

(Chair)

Patrick Pössel, Ph.D.

(Member)

Hongryun Woo, Ph.D.

(Member)

Ralph Piedmont, Ph.D.

(Member) 


\section{ACKNOWLEDGMENTS}

I would like to thank Dr. Leach for his guidance and support for the duration of this research project, as well as throughout my graduate career. I would also like to thank my committee members for their time, support and mentorship.

Special thanks to my family for their faith in me and unceasing support. To my beloved husband, who has been my rock and who offered emotional and financial support throughout the years of my pursuit of higher education. To my loving parents, who have been there for every step of this journey, and who went above and beyond in helping me gather research participants for my study. To my wonderful sisters, who celebrated my achievements with me and were always there for me in my moments of weakness.

Without your support and inspiration I would not be where I am today. 


\section{ABSTRACT \\ SPIRITUAL TRANSCENDENCE AS A SIXTH PERSONALITY FACTOR IN RUSSIA: CROSS-CULTURAL GENERALIZABILITY AND INFLUENCE ON PSYCHOSOCIAL OUTCOMES}

Ksenia Kopeikin

July 20, 2017

Previous research has suggested that Spiritual Transcendence (ST), which is the ability to stand outside one's own immediate experience, to see unity in nature and to feel connection with the rest of humanity and the spirits, is a universal human experience. Research also suggests that ST may form a sixth personality factor, operating in a manner similar to, but separately from the Big Five personality traits. That is, ST may be a source of intrinsic motivation and influence human behavior and psychosocial outcomes similar to the Big Five. Previous research has established the incremental validity of ST over and above the Big Five in predicting a number of psychosocial outcomes. The current study aims to investigate whether ST can be extracted in a previously untested culture, language and religion, and whether it predicts psychosocial outcomes over and above the Big Five. Additionally, this study seeks to investigate the protective nature of ST from stressful environments. It was hypothesized that ST will moderate the relationship between stressful environment, known as the neighborhood disorder, and the three outcome variables (personal control, generalized trust and psychological well-being) that were shown to be negatively affected by the neighborhood disorder. A sample of 256 Russian adult nationals was obtained using snowball sampling technique. All measures 
were translated into Russian. Results supported the concept that ST may be a universal human experience, as it was successfully extracted in a new culture, language and religion. ST also predicted one out of three outcomes over and above the Big Five personality factors, offering some support to the notion that ST may be a sixth personality factor. Finally, ST did not moderate the relationship between the neighborhood disorder and the outcomes. Future research exploring ST and its relationship with outcomes and the Big Five in Russia and in other cultures is encouraged. Further implications for research and counseling, and future directions are discussed. 
TABLE OF CONTENTS

ACKNOWLEDGMENTS ...............................................ii

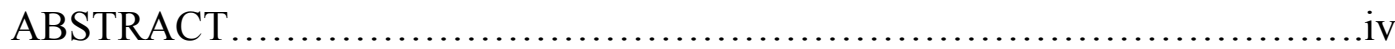

CHAPTER I: LITERATURE REVIEW .................................

Introduction........................................................

Purpose of Study..............................................

Spiritual Transcendence.........................................4

Spirituality in Russia.........................................17

The Five Factor Model........................................20

Five Factor Model in Russia.........................................25

Neighborhood Disorder.......................................29

Neighborhood Disorder in Russia...................................32

Personal Control.................................................. 34

Generalized Trust................................................38

Moderation Model..................................................42

Hypotheses..................................................46

CHAPTER II: METHOD ............................................. 50

Participants.................................................50

Measures..........................................................51 
Procedure.......................................................... 58

CHPTER III: RESULTS .............................................61

Preliminary Analysis..............................................61

Primary Analysis..............................................66

Supplemental Analysis.........................................81

CHAPTER IV: DISCUSSION ........................................... 83

Limitations.................................................92

Implications for Counseling...................................95

Future Directions...............................................97

REFERENCES..................................................... 101

CURRICULUM VITAE.............................................. 123 


\section{CHAPTER I: LITERATURE REVIEW}

\section{Introduction}

Psychological well-being is one of the most important psychosocial outcomes. Poor mental health often significantly and negatively affects life satisfaction, physical health and psychosocial functioning (e.g., Aro, Nyberg, Absetz, Henriksson, \& Lonnqvist, 2001; Fuller-Thomson, Agbeyaka, LaFond, \& Bern-Klug, 2016; Uma, 2016). Psychological well-being is important to address when evaluating individual differences, as it helps counselors and researchers to better understand which factors contribute to mental health and which factors might negatively impact it. For instance, exposure to stressful environment may have negative effects on psychological well-being. A construct known as the neighborhood disorder has been linked with higher levels of distress and poorer mental and physical health (Hill, Ross, \& Angel, 2005; Latkin \& Curry, 2003; Schieman \& Meersman, 2009; Schulz et al., 2000). Neighborhood disorder refers to communities with high levels of threat (both, perceived and actual victimization), scarce resources, and perceived lack of social order and control (Ross, 2000; Ross, Mirowsky, \& Pribesh, 2002).

A theoretical explanation to this connection between presence of neighborhood disorder in the immediate environment and lower psychological well-being is subjective alienation, which is a sense of separation from self and others (Mirowsky \& Ross, 2003; 
Seeman, 1983). There are two types of subjective alienation that have been empirically shown to connect the neighborhood one lives in with one's mental health - sense of personal control and generalized trust (Ross \& Mirowsky, 2009). The more disadvantaged the community, the lower one's sense of personal control over the events and trust in other people. These lowered factors in turn negatively affect one's psychological well-being, by increasing anxiety, anger and depression. Therefore, finding ways to increase a sense of control in residents of a disadvantaged neighborhood and increasing their trust in others may alleviate some symptoms of psychological distress resulting from daily living in a stressful environment.

Research into protective factors for mental health is growing (Bieda et al., 2017) and it is important to investigate individual differences that may contribute to better psychological health. One such protective factor may be spirituality. There is something unique and prevailing about one's faith that has a very special meaning in an individual's life. Religion can be a great source of coping and psychological well-being (e.g., Greenway, Phelan, Turnbull \& Milne, 2007; Pargament, Smith, Koenig \& Perez, 1998; Pargament, Koenig \& Perez, 2000). One's relationship with higher spiritual powers can provide a sense of unity, joy, support, consolation, purpose and meaning in life. Faith may offer a distinct and exceptional kind of solace that fellow human beings may not be able to provide for each other. Feeling connected to higher powers may be particularly beneficial when one faces stressful life events or personal difficulties (Ano, \& Vasconcelles, 2005; Pargament, Koenig, \& Perez, 2000). Often, individuals turn to higher powers only in times of deep distress or challenging situations and do not engage in religious or spiritual rituals otherwise (Pargament, Smith, Koenig \& Perez, 1998). 
When all else fails, connecting with the spiritual part of the world may offer hope and strength. One of the purposes of this study is to examine the relationship between one's spirituality and favorable psychosocial outcomes, namely feelings of trust, sense of personal control and psychological well-being, when exposed to the neighborhood disorder.

In addition to spirituality, personality provides another lens through which to look when investigating the relationship between stressful environment and one's psychological well-being. Similar to faith, one's personality can influence an individual's thoughts, feelings, behaviors, interpersonal relationships and coping mechanisms (e.g., Costa \& McCrae, 1980; McCrae \& Costa, 1986). Indeed, an individual's personality affects his or her perception of other people's motives and of events, which in turn influences one's thoughts, feelings, and behaviors. For instance, if an individual has aggressive or anxious personality traits, he or she may have a greater difficulty effectively communicating with others, finding sources of support, or reaching out to spiritual realms. On the other hand, an individual with prosocial or open-minded personality traits may be more open to the idea of reaching out for support, whether it is to higher powers or fellow human beings. Therefore, when studying individuals immersed in a stressful environment it is important to consider their personality traits, along with other potential sources of coping, such as spirituality. It is the intersectionality between spirituality and personality that may have the greatest effect on one's psychosocial outcomes.

\section{Purpose of the Study}


The first purpose of the current study is to examine the constructs of personality and spirituality in Russia, a country that has not been previously investigated. It is of interest to determine whether spirituality constitutes a separate domain from the personality factors as noted in the US and other nations and whether it offers any unique predictive validity over and above the traditional personality constructs of the Five Factor Model. The second purpose of this study is to investigate the effects of the neighborhood disorder on several psychosocial variables in Russians, and to examine how spirituality may moderate these effects. Research shows that neighborhood disorder leads to a variety of negative psychosocial outcomes, and has been associated strongly with lowered sense of personal control, generalized trust and psychological well-being (Hill, Ross, \& Angel, 2005; Mirowsky \& Ross, 2003; Ross \& Mirowsky, 2009). A premise of the current study is that an individual's spirituality may impact his or her psychosocial outcomes even when a stressful environment is constantly present. The findings may have implications for better understanding of the structure of coping mechanisms that operate to help individuals survive under stressful conditions.

In the introductory section that follows I will discuss spirituality and personality models utilized in this study, with each section including the discussion of these constructs in Russia. Next, I will describe the neighborhood disorder and its manifestation in Russia. Finally, I will discuss the three psychosocial outcome variables tested in this study, namely a sense of personal control, generalized trust and psychological well-being.

\section{Spiritual Transcendence}


Over the past several decades in the United States there has been a dramatic increase in interest toward the construct of spirituality, while attention towards religiousness has been decreasing (Weaver, Pargament, Flannelly \& Oppenheimer, 2006). In recent years, more research has begun focusing on investigating the construct of spirituality, although a large body of literature continues to be published on religiousness as well. Along with the rising interest in spirituality, there has been much confusion regarding the conceptual definitions and measurement of spirituality and religiosity (Slater, Hall \& Edwards, 2001). Many scholars use these terms interchangeably, arguing that they are equivocal, which adds to the confusion (Zinnbauer et al., 1997). However, Piedmont, Ciarrocchi, Dy-Liacco, and Williams (2009) have argued that while these two constructs share much overlap, they do represent different psychological qualities and thus these constructs may have very different meanings. Historically, religion has been defined as a broad construct, encompassing both individual and institutional components (Del Rio \& White, 2012). Various definitions of religiousness have been held by scholars and lay people. Some have defined it in a very concrete manner, solely referring to practices that a specific faith dictates (Doyle, 1992); others expressed more abstract definitions, such as "the inner experience of the individual when he [sic] senses a Beyond, especially as evidenced by the effect of this experience on his [sic] behavior when he [sic] actively attempts to harmonize his [sic] life with the Beyond" (Clark, 1958, p. 22). With the rise of secularism and disillusionment with religious institutions, spirituality has become gradually separated from religiousness at social and scientific levels (Zinnbauer et al., 1997). Perhaps the movement towards spirituality reflects larger social trend towards de-institutionalization and individualization (Pargament, 1999). As 
such, spirituality has received more attention in research over the past decades.

According to Zinnbauer and colleagues (1997), spirituality represents a more independent and individual aspect of one's relationship with higher powers. Spirituality has received little agreement in its operationalization among the scholars, but the existing definitions generally reflect the trend towards individualism. For instance, spirituality has been defined as an individual's relationship with God (Benner, 1989) and "a subjective experience of the sacred" (Vaughan, 1991, p. 105).

Today, religion is often viewed as a set of traditions, rituals, beliefs, and conventions of meaning that are related to one's culture (Burke et al., 1999), while beliefs and values associated with spirituality are not influenced by one's culture, but are inherently human. As Del Rio and White (2012) asserted, "human beings are not born religious; in contrast, they are born spiritual" (p. 133). That is, religion is a result of human spirituality. In line with this argument, one can be spiritual but not religious, yet one cannot be religious without being spiritual. Similarly, Piedmont, Ciarrochi, DyLiacco and Williams (2009) viewed spirituality as an individual attribute, one's personal relationship with larger, transcendent realities, while religiosity was considered to shape one's expression of spirituality in their community through a set of specific traditions and rituals.

Piedmont (1999) proposed a new construct termed Spiritual Transcendence (ST). The purpose of this new term was to operationalize spirituality as a broad, universal and non-culture-specific concept. ST relates not only to spiritual aspects of human existence, but to a larger source of intrinsic motivation, that may be expressed in ways such as altruism or nationalism. Piedmont (1999) defined ST as 
The capacity of individuals to stand outside of their immediate sense of time and place and to view life from a larger, more objective perspective. This transcendent perspective is one in which a person sees a fundamental unity underlying the diverse strivings of nature (p. 988).

Piedmont asserted that ST develops from an individual's efforts to create some sense of purpose and personal meaning for one's existence in this world. It refers to the ability to find unity with nature and connectedness with humanity, and is a source of intrinsic motivation that develops throughout the entire life. The concept of ST differs from other operationalizations of spirituality because it incorporates both Eastern and Western beliefs (Piedmont, 1999). At the very core, ST represents a domain of psychological functioning, expressed in one's level of commitment to spiritual entities and the degree of emotional support received from it. According to Piedmont (1999), ST is comprised of three components: a sense of connectedness, a belief that one is part of the whole humanity; universality, a belief in the unity and the purpose of life; and prayer fulfillment, a sense of joy and contentment that comes from engaging with the spirits through prayer or other rituals.

Piedmont (1999) proposed that ST is a separate psychological dimension, which can provide valuable new information about an individual in addition to what can be captured by other measures of individual differences. That is, ST offers a unique insight into the complex nature of human personality. Piedmont chose to study ST in the context of the Five Factor Model (FFM) of personality because he deemed it to be the only comprehensive organization of differences among individuals. Piedmont claimed that ST is distinct from the dimensions captured by the five personality factors, and that it 
constitutes a sixth personality factor. Furthermore, Piedmont argued that ST cuts across all five dimensions of FFM, and can influence the way one's personality broadly operates. For instance, it may influence our perspective on events and may affect the goals that we set in life or the way we interact with self and others. Another difference between ST and the five personality factors is the process of their development. According to Costa and McCrae (1994) personality is set by age 30 and does not change significantly after that. However, Piedmont argued that ST continues to evolve throughout the life span. With age, people begin to see larger patterns in life and human nature, and getting closer to one's death frequently triggers the need to understand the meaning of one's life. Therefore, older individuals may in fact have a more developed sense of ST.

In his pioneering study, Piedmont (1999) analyzed two distinct large samples of U.S. undergraduate students, who predominantly identified with some type of Christian religion (98\% and $88 \%$ ). The participants completed the questionnaire themselves, and then asked two acquaintances to rate them on the same measures. The questionnaire included a variety of psychosocial measures, such as perceived social support, vulnerability to stress and overall well-being, as well as measures of FFM and ST. The initial factor analysis extracted six factors that explained $57 \%$ of the variance. The items of the FFM assessment each aligned with their intended factor, while the items of Spiritual Transcendence Scale (STS) aligned to form their own factor (with factor loadings above .55). This finding suggested that ST represents a separate sixth factor distinct from the FFM. Moreover, the analysis demonstrated statistically significant $(p<$ .05) predictive validity of ST for a number of psychosocial factors. These results 
indicated that ST does affect certain characteristics of individuals that lay people frequently consider to be part of one's personality, such as one's attitudes and perceptions. That is, ST is not specific to defining a person's expression of religiousness and spirituality, but influences an individual in a variety of psychological domains, such as interpersonal orientation and perceived social support. As such, it can theoretically be considered a part of one's personality. This notion is further supported by the fact that a separate factor structure of ST was extracted from observer ratings as well. Therefore, based on this data it can be concluded that aspects of ST are not merely a private psychological phenomenon, but are readily observable by others, similar to the five factors of personality.

Furthermore, Piedmont discovered that STS items provided an additional and statistically significant explanatory power, over and above the five personality factors. It is important to note, however, that only the Prayer Fulfillment subscale added explanatory power for all outcome variables assessed in the study, including perceived social support, prosocial behavior, sexual attitudes, vulnerability to stress, attitudes toward abortion, interpersonal orientation, sense of personal control over one's health and faith maturity. The Universality facet contributed only to sexual attitudes, while the Connectedness facet helped explain only perceived social support and prosocial behavior. This finding may indicate that the Universality and Connectedness facets of STS share more conceptual basis with the five factors of personality than the Prayer Fulfillment facet, and therefore explain less unique variance over and above the FFM. Perhaps the Universality and Connectedness facets could be more similar to such factors of personality as Extraversion or Agreeableness than is Prayer Fulfillment. For instance, 
sense of connectedness with others may be related to one's extroverted nature and sociability. Similarly, one's belief in the unity and purpose of life may be related to one's prosocial tendencies that orient an individual towards others and intrinsically motivate an individual to engage in moral behaviors. It is also important to consider that Piedmont's sample was predominantly Christian. Prayer Fulfillment facet may be more influential for this group, but this finding may not hold true for other religious or spiritual groups. Another possible explanation for finding that Universality and Connectedness facets added less explanatory power to the outcomes than did Prayer Fulfillment facet may be that because Piedmont's participants were young adults, they simply had not developed a strong sense of Universality or Connectedness yet, as those constructs require life experience and reflection, whereas prayer may be taught early in life and is conceptually easier to grasp. Therefore, the Universality and Connectedness facets of STS were not as influential in predicting psychosocial outcomes for college students as did Prayer Fulfillment.

Piedmont replicated his findings in a later study (Piedmont, 2001). In that study he administered the STS to 322 U.S. undergraduate students, who again predominantly identified with some type of Christian faith (89\%), with less than $1 \%$ identifying as Jewish and the remaining $10 \%$ indicating some other religion. Self and observer ratings were obtained on a number of psychosocial measures, such as affect and prosocial behavior, as well as on measures of FFM and ST. Similar to the initial study, the STS items loaded on their intended facets, forming a sixth factor separate from the FFM factors. Together, the six components explained $58 \%$ of the common variance. The overall internal consistency of the self-rated STS was high (alpha coefficient of .87 for 
the total scale). Similar to earlier findings alpha coefficients for the Universality and Prayer Fulfillment facets were high (alpha coefficients of .82 for both facets), with the exception of the Connectedness facet, which was low (alpha coefficient of .58). All three of the STS facets were significantly correlated with the psychosocial outcome variables tested in this study, although the Prayer Fulfillment facet had the highest correlations, while the Connectedness facet generally had the lowest. Again, these results replicate the findings of the original study, demonstrating a larger influence of the Prayer Fulfillment facet on psychosocial outcomes. Furthermore, Piedmont demonstrated the incremental validity of the STS over and above the FFM, with STS facets adding from $11 \%$ to $50 \%$ of personality's contribution to variance. Different facets of STS predicted different psychosocial outcomes. For instance, Prayer Fulfillment predicted self-actualization, purpose in life and global well-being, while Universality predicted positive affect and affect balance, and Connectedness predicted prosocial behavior. Thus, all facets of STS contributed to predicting at least some psychosocial outcomes over and above the five personality factors, indicating the overall incremental validity of the STS.

It was later proposed that ST not only comprised a sixth personality factor, but also is a universal human experience, similar to the FFM, that can be found in other cultures and religions (Piedmont \& Leach, 2002). There is a consistent thread of religious and spiritual teachings and practices in every culture throughout the world and throughout time. When comparing religions across the world, there are many differences in the specifics of the teachings and rituals. For instance, Eastern cultures believe that all living beings have a soul, while Western religions make sharp distinctions between humans and the rest of the natural world. Eastern religions, such as Buddhism and 
Hinduism emphasize only one reality, while Judaism, Islam and Christianity promote the idea of dualism, which involves two realities, material and non-material. The process of enlightenment also is perceived differently in Eastern and Western cultures, with Eastern religions teaching that the source of enlightenment is within the individual and can be achieved by quieting the mind, while Western religions emphasize the importance of developing and maintaining a personal relationship with God.

However, there are many similarities among religious traditions as well. In all cultures, religion calls individuals to realize the limitedness of their perspective, to adopt a larger perspective on life and human existence across time and place, and to acknowledge the presence of the divine being that binds all things into a unified harmony. Because these fundamental teachings are mostly captured by the concept of ST, it is plausible to suggest that the three facets of ST can be found in other cultures or religious groups across the globe. Unlike other measures of spirituality, the STS was developed to reflect both Eastern and Western perspectives on religion. When Piedmont was developing his scale, he consulted a number of theological experts from various religions, including Buddhism, Hinduism, Quakerism, Lutheranism, Catholicism, and Judaism. Therefore, the construct of ST is expected to be applicable to a variety of cultures and religious teachings.

This hypothesis has been tested in a number of cross-cultural studies. For instance, Piedmont and Leach (2002) evaluated ST in a sample of undergraduate students in India, in an attempt to understand whether the construct of ST is generalizable to nonChristian and non-Western cultures. Of these participants, the majority identified as Hindu, with Christians and Muslims comprising a third of the sample. They were 
administered a questionnaire similar to those used in previous studies, measuring the FFM, ST and a number of psychosocial outcomes (e.g., purpose in life, affect). The results of the study provided strong support for the presence of Universality and Prayer Fulfillment facets of STS in Indian culture, although alphas were lower than in the original US sample. However, the alpha coefficient for Connectedness facet was much lower than for the other two subscales of STS. In the American sample the Connectedness facet had the lowest alpha coefficient as well, but it was much higher than that in the Indian sample. Furthermore, the model fit was significantly better after the Connectedness items were deleted. This finding suggested that the Connectedness facet may not be very meaningful in Indian culture, or at least among the Indian undergraduate students. Perhaps college students felt less connection with those around them as they were going through the transition of building an independent life and separating from their families. Since there is generally more value placed on one's family in collectivistic cultures such as India (Verma \& Triandis, 1999), the growing independence associated with college life may have contributed to low internal consistency in the Connectedness facet.

In addition to establishing ST as a valid construct in India, Piedmont and Leach also established its incremental validity over and above the five personality factors. Again, the Prayer Fulfillment subscale was the most robust predictor, with Connectedness subscale predicting only frequency of reading the scripture, and both Connectedness and Universality facets predicting only faith maturity. This finding offers further support to the notion that Prayer Fulfillment facet of STS has particularly strong incremental validity over the FFM. 
Interestingly, Piedmont and Leach (2002) also found a moderation effect of religion and gender on the expression of ST. In their study, Christian and Muslim women scored significantly higher on religious affiliation than their male counterparts, with Christian women scoring the highest and Muslim men scoring the lowest out of the entire sample. A closer examination revealed that there was a significant gender difference only within the Universality facet of STS, with women scoring higher than men. Furthermore, religion significantly affected the manifestation of ST, with Christians scoring significantly higher on Prayer Fulfillment, and Muslims scoring significantly lower on Universality facet than the other groups. Taken together, these results suggested that while ST appears in different cultures and religions, the manifestation of its specific facets varies among gender and religious affiliations.

In the majority of the studies, the STS has been examined in the language in which it was developed, American English. Establishing the presence of the STS in another culture and another language would further the argument of the universality of the ST, since concepts that are important in a given culture have native words to describe them. Therefore, if the construct of ST still holds after being translated into another language, it would arguably demonstrate the importance of ST in that culture. Piedmont (2007) investigated whether the construct held in the Philippines, after being translated into Tagalog, a native Filipino language. He administered both the translated STS and the English version of STS to two samples of Filipino adults. About $90 \%$ of both samples identified as Roman Catholic, with the remaining 10\% representing other Christian faiths. Self and observer ratings were collected on a number of psychosocial variables, such as prosocial behavior and general well-being, as well as the FFM and ST. Participants in the 
second sample were randomly assigned to complete the STS twice, either in the same language both times (Tagalog or English) or in two different languages.

Overall, the results of the study evidenced the presence of ST in the Filipino culture. In fact, the mean scores of the STS were about eight points higher than those for the American sample, indicating a more robust presence of ST in the Filipino culture than in the United States. Piedmont speculated that perhaps this finding was due to the difference in participants' age, as the Filipino sample included adults, while the American sample included college students. Because ST continues to develop throughout life, it can be expected that higher and more stable levels of ST would be found in an adult sample than in a college sample. Similar to the Indian sample, alpha reliabilities of self-reported ST were lower than those found in the United States. Again, the Connectedness facet showed the lowest reliability. The same structure of STS was established for both the English and Tagalog versions, with the STS items falling within their intended facets, and representing a single dimension in Filipino culture, similar to findings in the American and Indian samples. Furthermore, the STS was significantly correlated with all outcome measures, indicating that in Filipino culture ST is associated with a number of psychosocial factors, similar to those found in the United States and India. In line with the previous findings, the STS provided a significant additional explanatory power over and above the five personality factors for both self- and observer-ratings. Finally, the testretest correlations for the Tagalog-only STS version were generally higher than for the English-only version, suggesting that the translated STS was more stable and perhaps better understood by the respondents. This finding may indicate the need to translate the 
STS into the native language of the country where it is administered, for more accurate results.

The majority of variance in outcome ratings was explained by the Universality facet of the STS, contrary to results found in the United States and India, where Prayer Fulfillment facet was the most robust predictor over and above the FFM. Perhaps these results can also be explained by the difference in age of the participants. It could be argued that at a younger age Prayer Fulfillment is more influential and affects psychosocial outcomes more. As individuals age and gain more life experience, they begin to see the larger picture, the universal patterns of life and human nature, and develop a better sense of their purpose in life. Therefore, with age the Universality facet of STS may have larger influence on an individual than other facets.

In his article, Piedmont (2007) also reviewed unpublished dissertations, theses, and conference papers that provided further support to the overall presence of ST factor in other cultures. For instance, he reported that Goodman (2002) evaluated American conservative, orthodox and reformed Jews, while Wilson (2004) studied ST in aboriginal Canadians. According to Piedmont (2007), both researchers found the reliable and valid presence of ST in their samples. Furthermore, Cho (2004) translated the STS into Korean and found it to be a valid predictor of relationship dimensions in a married Christian sample, while Bourdeau, Hinojosa, Perez, and Chu (2004) translated the scale into Spanish for use with Latino men and found that the scales capture native Meso-American religious themes not found in Christian based religions (as cited in Piedmont, 2007). Taken together, these findings offer support to the claim that ST is a potentially universal and unique factor that exists in many cultures and religions. 


\section{Spirituality in Russia}

Further examination of ST in other cultures and religions is needed to add to the understanding of ST as a valid cross-cultural variable. In his review of Piedmont and Leach's (2002) study Otani (2002) argued that in order to demonstrate the universality of ST, a variety of languages and religions would need to be evaluated. Russia presents a unique opportunity to study a culture that has been influenced by both Eastern and Western traditions, due to its geographic location and vast size. It represents a diverse population of Asian and European descents, with 22 different nationalities living on the territory of the Russian Federation (Russian Census of 2010, http://www.perepis2010.ru/). A Russian sample can provide a variety of cultural backgrounds, and add to the understanding of the influence of ST in other parts of the world.

In addition, conducting a study in Russia presents an opportunity to test the universality of ST in a language and culture that has not been previously studied. The Russian language is formed by the Cyrillic alphabet, which is derived from a different language family than those that have been previously studied. Languages in the previous studies included English and Spanish, which are formed by the Latin alphabet; Tagalog, which originates from the Astronesian language family; and Korean, which is considered a language isolate. Analysis of language is important because a lexicon represents the culture and what is important for its people. Any concept that carries high value, survival mechanism or adaptive usefulness for that culture has a native word for it. If evidence of ST were to be found in a country with a language originating from a different etymological root than that of other cultures studied, it would offer further support to the theory of the universality of ST. That is, establishing a similar concept of ST in several 
cultures using different language systems would arguably demonstrate that this concept represents an important experience for all of these cultures, and therefore may contribute to our understanding of it as a universal construct. Therefore, studying ST in Russia presents an opportunity to assess ST in a different language family.

Furthermore, studying ST in Russia presents an opportunity to test the manifestation of this construct in a different religious setting. Although the Russian Orthodox religion originates from Christianity, it is different from Christian religions found in the United States, such as Catholicism, Protestantism, and Evangelicalism. According to Father Michael Azkoul (http://www.ocf.org/OrthodoxPage/reading/ortho_cath.html) one of the most significant differences between Russian Orthodoxy and Roman Catholicism is that Roman Catholicism endorses a theory of "doctrinal development," the idea that Christ only gave people the "seed" of faith and that it has been growing, maturing and changing throughout centuries. Russian Orthodoxy, on the other hand, endorses the view that the teachings of Christ should not be changed. Consequently, since 988 A.D. when Russians were introduced to Orthodoxy and baptized, none of the aspects of the faith have been altered, and Orthodox Christians today claim to believe exactly the same doctrines as Orthodox Christians of the first centuries. As Carevsky (1898) argued in his book, because of this unchanging faith over the centuries, Orthodoxy has deeply affected the formation of the Russian culture. With the introduction of Christianity began the development of literacy, art, music, and the government itself. According to Carevsky, Christianity became so intertwined with the Russian culture that the two became inseparable, and spirituality became one of the defining aspects of Russian national 
character. This notion is also evident from the rapid revival of Russian Orthodoxy after the collapse of the Soviet Union, which suppressed religion for 74 years. Even during the Soviet Union, when the whole country was required to be atheist, millions of Russians held on to their beliefs in private (Ardichvili, 2006; Froese, 2004).

Today, most ethnic Russians still view the Orthodox Church as a vital part of Russia's national identity (Ziegler, 2008). According to studies conducted by Russian Public Opinion Research Center, $75 \%$ of the Russian population identify as Russian Orthodox (http://www.wciom.com/index.php). However, the spiritual qualities of individuals are more important to most Russians than is religiousness. An average Russian does not frequently attend church (Ziegler, 2008), but Russians consider themselves very spiritual. In the $19^{\text {th }}$ century, in an attempt to separate themselves from the industrialized and materialistic Western cultures, Russians began actively defining themselves in terms of their spirituality, their distinctive "Russian soul" (Allik et al., 2011). This term has become the central construct in describing the Russian national character. Influential novelists and historians have used this term to explain Russia's cultural development, its history and its place in the world. For instance, a well-known linguist, Anna Wierzbicka, identified three words that seem to best encompass Russian mentality: dusha ("soul"), sud'ba ("fate"), and toska ("melancholy"; Allik et al., 2011). These words relate to the idea of transcendence in Russian culture, by highlighting the experiences beyond the physical, present-day level. Many themes in the Russian culture are linked to the concept of the Russian soul. These themes emerge in Russian literature, common sayings and expressions, rituals and superstitions. It has become a cultural norm to endorse spiritual values in everyday life (Figes, 2003). The influence of Orthodoxy on 
Russian culture has been so strong that even non-believers use metaphors (e.g., proverbs), symbols, and behavior norms propagated by the Orthodox religion (Ardichvili, 2006). Therefore, it is expected that the evidence of ST will be robust in a Russian sample, given the strong influence of spirituality on the Russian culture.

This study will investigate the presence and manifestation of ST in the Russian culture. However, in order to establish whether ST can be considered a part of the Russian personality, the Five Factor Model will be included as the basis for understanding the Russian personality. If ST shows predictive power over and above the five factors of personality, it will be considered a unique, sixth factor of personality in the Russian culture. This will also provide further evidence for the claim of the universality of ST.

\section{The Five Factor Model}

One of the most influential and dominant theories of personality is the Five Factor Model (FFM; e.g., Costa \& McCrae, 1992). The origins of the FFM can be traced back to the 1930's, when McDougal (1932) and two German psychologists, Klages (1926) and Baumgarten (1933) first suggested that personality can be categorized into five factors by carefully analyzing and organizing the language (Digman, 1990). The rationale behind organizing the language, known as lexical hypothesis, involves the idea that people have invented words to describe those personality traits that are important in human life. Therefore, a factor analysis of the trait lexicon would, in theory, reveal the comprehensive personality structure. Systematic efforts to identify consistent personality factors were undertaken by Cattell in the 1940's. Cattell identified thousands of terms that potentially described consistent and stable traits of personality. He then condensed 
the initial set of terms by combining semantic judgment with correlational and factor analyses over a decade of work. However, his research yielded inconsistent results that were difficult to replicate. It wasn't until the 1960's that Tupes and Christal (1961) and Norman (1963) found five recurrent personality factors in the analyses of 12 different samples of male college students and graduates. Their research, however, remained largely unknown to personality psychologists for a variety of reasons (see Digman, 1990). It did not re-emerge until the 1980 's, when a number of researchers conducting careful analyses concluded that five personality factors are indeed fundamental dimensions of personality, found in self-reports and ratings, and in different age groups, genders, occupations, and nations (John, 1990).

The FFM is a taxonomy of personality traits, which are "dimensions of individual differences in tendencies to show consistent patterns of thoughts, feelings, and actions" (McCrae \& Costa, 2003, p. 25). Theoretically, FFM includes five broad personality traits that can be used to classify individuals. The five personality traits are labeled Openness to Experience, Conscientiousness, Extraversion, Agreeableness and Neuroticism (McCrae, Gaines, \& Wellington, 2013). Openness to Experience is the least studied factor to date. High scores describe people who are open-minded, curious and seek out new experiences, while low scores describe people with a conservative, traditional and dogmatic approach to life. Individuals scoring high on Conscientiousness traits are efficient, organized and self-disciplined, and those on the lower end tend to have difficulty organizing their behavior to achieve their goal. Furthermore, high scores on Extraversion indicate sociability, cheerfulness, and love for fun, as low scores describe reserved, unadventurous and somber individuals. Agreeableness refers to prosocial traits, 
such as honesty, forgiveness, generosity and cooperation, or on the opposite end hostility, selfishness and arrogance. Finally, Neuroticism describes the tendency to experience distressing emotions such as anxiety, depression, resentment and guilt. Individuals with low scores on this trait are emotionally stable, confident and resilient.

Cross-cultural studies of the FFM have demonstrated the adequacy of the five factors in broadly describing individual personalities across the globe. For instance, in his meta-analysis Rolland (2002) compared 16 factor structures extracted from diverse cultures (including Russia) using the Revised NEO Personality Inventory (NEO-PI-R), a questionnaire specifically designed to assess the five personality factors. For his analysis, Rolland utilized a Tucker's (1951) congruence coefficient index, which is widely used in factor analytic studies that attempt to replicate previous findings across different samples (as cited in Rolland, 2002). The congruence coefficient can be used to study the similarity of extracted factors across different samples, or in this case across different cultures. High congruence coefficient for a given personality factor would indicate its generalizability across cultures. The factorial replication threshold is set at .85 (Haven \& ten Berge, 1977 as cited in Rolland, 2002).

The results yielded coefficients of congruence above .85 for all cross-cultural comparisons for Neuroticism and Conscientiousness factors, which indicated crosscultural validity of these two dimensions. Out of 30 coefficients of congruence 28 reached the .85 threshold for Openness and Agreeableness, with the other two slightly above .80. Similarly, Extraversion factor showed coefficients of .85 or above for all cross-cultural comparisons, except for four, which still had coefficients of .80. Rolland (2002) also found that certain facets of Agreeableness (e.g., trust, altruism, compliance) 
and Extraversion (e.g., warmth, assertiveness, excitement) dimensions varied more between Eastern and Western cultures, presumably because they relate to interpersonal aspects of one's personality. For instance, assertiveness is encouraged more in the Western cultures, while Eastern cultures emphasize the value of compliance and humility. Similarly, expression of excitement, affection and warmth looks different in different parts of the world. Overall, the use of the FFM was deemed justifiable in crosscultural research, although Rolland cautioned against using it as the optimal representation of all cultures.

In another study, McCrae (2002) investigated the generalizability of the NEO-PI$\mathrm{R}$ to 36 different countries (including Russia) across different age and gender subsamples. He found the same patterns of personality differences for different gender and age groups that were established previously (e.g., Costa \& McCrae, 2002). For instance, women scored higher than men on the Neuroticism, Openness and Agreeableness factors. Similarly, young adults across countries scored higher in Neuroticism and Extraversion and lower in Conscientiousness than older adults, who scored lower in Openness and higher in Agreeableness. The most pronounced variability emerged among subsamples in the European group, with Americans scoring in the middle, and the smallest variability being observed in Asian countries (except for China) and two Black African countries (i.e., South Africa and Zimbabwe). Thus, it appears that self-reported personality traits have larger variability in the European countries, while Asian countries showed less extreme response options. McCrae (2002) speculated that this finding may be due to problems in translation, avoidance of the use of extreme response options by Asians who value humility and reservation, or other cultural 
influences, such as a lower emphasis on individual traits in collectivistic cultures. These results may also reflect an accurate distribution of traits, with more homogeneity in personality in Asian and African cultures than in European and American groups.

Later, McCrae and colleagues (2005) investigated the cross-cultural structural equivalence of the five factors based on the observer ratings, as opposed to self-report measures that were previously used. The sample included college students recruited from 50 countries (including Russia) representing six continents, with many of the included cultures being studied for the first time (e.g., Arabic and several African). Participants were asked to rate another individual in one of four target conditions (college-aged women, college-aged men, adult [i.e., over 40 years old] men, or adult women) using the NEO-PI-R. The results yielded similar factor structures across the 50 countries, replicating the FFM structure within each of the four age and gender target groups. However, some discrepancies were notable. For instance, particularly low alphas emerged for the Openness factor in some African cultures, suggesting that for them it may not be a meaningful construct. Also, Agreeableness and Conscientiousness factors accounted for a larger amount of variance than what is observed in self-report data, a phenomenon that has been previously established. Perhaps these qualities are more readily observable by others than those associated with the other three factors. Finally, similar to previous findings (i.e., McCrae, 2002) women scored higher than men on all five factors. Perhaps this gender difference could be explained by generally higher emotional expressiveness by women, since men tend to be socialized to avoid displays of emotion in many countries. Overall, the researchers determined that the universality of 
the FFM in observer ratings has been confirmed, due to the majority of the countries showing the same factor structure of FFM as that found in the United States.

\section{Five Factor Model in Russia}

Although a variety of countries were extensively studied in cross-cultural analysis of the personality traits, some countries were examined less than others. Typically, the cross-cultural research is conducted based on convenience, such as cost and availability of research participants. Consequently, more developed countries, such as United States, Canada and Japan tend to be overrepresented in such research studies, while countries where the science of psychology is less developed are underrepresented (Allik et al., 2009a). One such underrepresented country is Russia. Only a few studies have investigated the personality structure of Russians (Slobodskaya, 2007). Existing literature suggests that the FFM structure is valid in Russian culture in self and observer ratings of personality traits (Martin, Costa, Oryol, Rukavishnikov, \& Senin, 2002; McCrae et al., 2005). Furthermore, Allik and colleagues (2009a) found similar gender differences in a Russian sample to those established in cross-cultural research, such as higher scores on Neuroticism, Openness and Agreeableness with women than with men. They also found that personality development throughout the life span in Russia follows the same pattern as in other countries, such as the Portugal, South Korea and United States.

Although Russians seem to have similar personality traits to those of other nationalities, some interesting findings have emerged when comparing ingroup and outgroup ratings of Russians. While there appeared to be a strong agreement between ingroup and outgroup ratings of Americans (e.g., Terracciano \& McCrae, 2007), studies have found that other countries view Russians very differently from how Russians view 
themselves. That is, although images of Americans, for example, are consistent throughout the world, it appears that Russians have a different self-image from how the rest of the world views them. For instance, Peabody and Shmelyov (1996) found that samples from European countries (i.e., Austria, England, Finland, France, Germany and Italy) viewed Russians as disciplined, serious and conscientious, while Russians viewed themselves quite opposite, as impulsive, impractical and lazy.

A later study by Realo and colleagues (2009) investigated how six countries neighboring Russia described themselves and a typical Russian, in an attempt to understand whether the national character stereotypes are true. The results indicated that all six countries shared the same beliefs about Russian national character, but these did not converge with Russians' self-rated characteristics, or with previous findings of Westerners' ratings of Russians (e.g., Boster \& Maltseva, 2006). It appears that neighboring countries of Russia not only viewed Russians differently from how Russians view themselves, but also differently from how the rest of the world views them. This indicates an inconsistent image of Russian national character throughout the world, an interesting finding that is difficult to explain. Perhaps the Soviet propaganda affected how the rest of the world views Russians, although these popular depictions were not true for the majority of Russia's nationals. It may be argued that neighboring countries had more opportunities to meet representatives of Russian culture, but due to constant struggle for resources and independence have formed a different opinion of Russians, distinct from that of the rest of the world.

Conversely, Allik and colleagues (2009b) found that within the Russian borders the image of a typical Russian appears to be relatively consistent throughout the country, 
despite its large size and differing regions. One commonality found in their research, a most salient characteristic that distinguished Russians from other nations, was their openness. That is, a typical Russian was portrayed as someone who has intellectual curiosity, a willingness to examine their own values, and a vivid imagination and fantasy life. A similar portrait was established in the earlier study (Terracciano et al., 2005). Another study found that older Russian men and women scored higher on the Neuroticism scale, as compared to younger Russians and Americans of all age groups (Martin et al., 2002). Neuroticism scores tend to decrease with age in other countries that were previously investigated (Costa et al., 2000), suggesting a personality development pattern that may be unique to Russians. This pattern may have been observed in older participants in this particular study due to dramatic political and economic changes in the country over the past several decades, which may have increased these individuals' neuroticism. Conversely, this finding may represent a unique aspect of Russian culture and personality changes associated with age.

It is evident that the view of a typical Russian is different across the world. Russia continues to be a mystery for many, including Russians themselves. According to Berdyaev (1960), inconsistency has been claimed to be a characteristic of a typical Russian (cited in Allik et al., 2011), which perhaps interferes with Russians' own ability to precisely describe their national character. One of the most famous quotes frequently used by Russians today to describe themselves is that of their great poet, Fyodor Tyutchev (1803-1873), “Umom Rossiyu ne ponyat” (“One cannot understand Russia by reason"). Similarly, Winston Churchill famously described Russia as "a riddle wrapped inside a mystery inside an enigma" (Chambers Dictionary of Quotations, 2005, p. 216, 
cited in Robertson, 2006). All in all, it appears that not all aspects of the Russian culture can be captured with traditional measures of personality. Perhaps the missing piece is the distinct Russian soul, an enigmatic construct that has not been successfully measured yet. It may be that the STS may offer further insight into the Russian personality, by highlighting the spiritual aspects of this culture that are so important to Russians and have shaped their culture in so many ways. The spiritual aspect of Russian personality has not been emphasized in any of the previous studies.

In order to establish predictive validity of ST over and above the FFM, a number of psychosocial outcome variables need to be examined in addition to measures of FFM and ST. Many different psychosocial factors are relevant to the Russian culture. However, when considering those that may affect the personality and the soul of a Russian the most, environmental factors may be particularly influential. Russia has stereotypically been associated with a harsh way of life and indeed, living in Russia can be very difficult and distressing. High crime rates, a poor economy and corruption contribute to the sense of powerlessness and despair in the country. These circumstances undoubtedly affect individuals' mental health. This study will investigate the relationship between personality traits (including ST) and the psychological functioning of a typical Russian, living in such demanding and difficult setting. It may be, for example, that high levels of spirituality, in addition to personality factors, help Russians to better cope with stressful circumstances. Therefore, in addition to investigating the spirituality and personality traits of Russian natives, another purpose of this study is to investigate the moderating effects of ST on the relationship between stressful environment, which can be characterized in terms of a neighborhood disorder, and psychosocial outcomes. 


\section{Neighborhood Disorder}

Pearlin and colleagues (1981) asserted that distress originates in the social world, meaning that the environment the individual lives in affects one's psychological health. That is, individuals who reside in areas with high levels of threat and scarce resources tend to experience higher levels of distress. Such areas are described by researchers as having a neighborhood disorder (Ross, 2000), which refers to "conditions and activities, both major and minor, criminal and noncriminal, that residents perceive to be signs of the breakdown of social order" (Ross, Mirowsky \& Pribesh, 2002, p. 68). At the very core it is the perceived lack of order and social control in the community. Perception of order is created through visible cues that residents observe in their neighborhood, such as noise, vandalism, graffiti, public drinking, disrepair and litter, as well as other crimes such as property theft. These give the perception of a potential threat. Major crimes are frequently unseen and fall on the extreme end of the disorder, but smaller indications of disorder are constant and harmful. In addition to experiencing such stressful environment on a daily basis, actual victimization may also happen to some individuals in the neighborhood, which affirms the reality of the perceived threat that residents experience daily and adds to the sense of an uncontrollable and disordered environment. That is, neighborhood disorder signals to its residents the potential for harm, while victimization indicates an actual threat (Ross, Mirowsky \& Pribesh, 2002).

Stress, victimization, and threat are frequently more common in neighborhoods that are high in crime and low in resources. Living in the areas where streets are dirty and dangerous, people drink alcohol and use drugs in large amounts, and police are not trusted creates emotional and psychological distress. Evidence is accumulating that 
disordered and stressful environments have negative effects on a variety of psychosocial and health factors. For instance, Steptoe and Feldman (2001) showed that neighborhood problems, characterized by factors such as traffic density, pollution, dirt, noise, absence of local facilities and amenities, and perceived threats to self and property, are associated with poor self-rated health reports, psychological distress and impaired physical functioning associated with the activities of daily living. Furthermore, Hill, Ross and Angel (2005) found that the relationship between neighborhood disorder and health is mediated by psychological and physiological distress. That is, chronic stressors in the immediate environment, such as crime, vandalism, drug use, garbage and noise, lead to the stress response in the body, in the form of psychological issues such as anxiety and depression, and physiological symptoms associated with the arousal of the autonomic nervous system, such as trouble breathing, nausea and upset stomach, chest pains and weakness. Chronic stress also reduces resistance to infection and cancer, as stress hormones released during stress response undermine the immune system in the long term. Thus, being exposed daily to mentally and physically distressing environments in disordered neighborhoods may erode one's physical health, in addition to negatively affecting psychological well-being.

A growing body of literature also evidences a connection between neighborhood disorder and psychological well-being. It was previously thought that disadvantaged neighborhoods contain disadvantaged residents, and that the connection between such neighborhoods and lower psychosocial outcomes of people living there is due to the demographic characteristics of the residents themselves, not the effects of the neighborhood per se (Jencks \& Mayer 1990; Robert 1998; Slogget \& Joshi 1994). 
However, Ross (2000), in a multilevel study, demonstrated that the neighborhood one resides in affects one's psychological well-being (particularly depression) over and above one's individual characteristics that could influence mental health, such as the amount of drinking, engagement in illegal activities, education, income and employment status. Yet, it is important to note that although all residents of disadvantaged neighborhoods are negatively affected by their environment, individuals who are considered more disadvantaged, such as women, the unemployed or unmarried individuals and those who have lower education and income, show greater levels of depression than individuals more advantaged, such as men, the employed, older, married, educated individuals with higher income. Therefore, individual differences are also important to consider, although neighborhood disorder significantly and negatively impacts everyone who resides in that area.

Research shows relationships between neighborhood disorder and other psychological issues, besides depression. For instance, Schultz and colleagues (2000) demonstrated lower life satisfaction and higher psychological distress in residents of high poverty areas. Similarly, Schieman and Meersman (2009) found positive relationships between neighborhood disorder and anger, anxiety and depression, in both men and women. A stressful and disordered environment negatively affects not only adults, but also children. In one study, neighborhood disorder was associated with higher instances of depression, anxiety, Oppositional Defiant Disorder and Conduct Disorder in children (Aneshensel \& Sucoff, 1996). Overall, a large body of literature points to the harming effects of neighborhood disorder on its residents' psychological and health outcomes. It is 
important to find ways to buffer the negative effects of the neighborhood disorder in order to preserve the residents' physical and mental health.

\section{Neighborhood Disorder in Russia}

It can be argued that modern Russia has a neighborhood disorder in many if not most of its geographic areas. It can be considered a disadvantaged community based on many different factors. After the collapse of the Soviet Union in 1991, Russia experienced drastic social, political and economic changes. The Russian government changed from totalitarianism to the democratic system, and the economy moved from socialism to capitalism. These transformations have impacted the country in many negative ways. In the decade following the collapse, the economic situation in Russia was worse than anything experienced in the United States, even during the Great Depression (Heleniak, 1995, as cited in Pridemore, 2002). Over 30 percent of the population lived below the poverty line, the unemployment rate rose more than 2.5 times and the gap between the social classes widened, with the top 20 percent of the population receiving almost 50 percent of the overall income in the country, while the bottom 20 percent received only six percent (Pridemore, 2002). Today, the economic situation in Russia is improving, but high levels of poverty and gaps between social classes remain, and it will be many years before the country sees true economic stability.

The economic struggles also led to a sharp increase in crime and violence in the country. In 2000, Russia had a homicide victimization rate of approximately 30 per 100,000 people, almost five times higher than the homicide victimization in the United States for that year (Kim \& Pridemore, 2005). Russia also had one of the highest homicide rates in the world through the transitional period of 1990s (Pridemore, 2003). 
Throughout the last decade high crime rates continued. In 2011, Russia had the second largest incarcerated population in the world, yielding only to the United States (World Prison Brief, 2011). In addition, property crime evidenced a sharp increase as well. For instance, in 2001 the rate of armed robberies in Russia was 2.5 times higher than that in the early 1990s (Kim \& Predimore, 2005).

Along with the rapid rise in crime, Russia experienced an increase in police corruption, which added to the sense of powerlessness in the country. Following the collapse of the Soviet Union, the Russian police force had assumed a predatory approach towards the citizens, and police violence had become a common occurrence. For instance, in 2004 a poll in large cities revealed that 25 percent of respondents had been beaten or tortured by the police ('Poll: 25\% Victimized by Police,'” The Moscow Times, 21 May 2004, p. 3 as cited in Gerber \& Mendelson, 2008). In their literature review, Gerber and Mendelson (2008) noted that Russia has one of the highest rates of police corruption, with issues such as bribe-taking, drug dealing, registering stolen cars and protecting members of the gangs. They also found that Russians have little confidence in their law enforcement and legal institutions, with only a quarter of the adult population stating that police can "probably" be trusted. Such low faith in the justice system only adds to the sense of social disorder and lack of personal control in the country.

Further evidence of disordered community in Russia is its high rate of suicide. Suicide is one of the leading causes of premature deaths in Russia (Nemtsov, 2003; Pridemore \& Spivak, 2003). In 2005, the suicide rate was twice as high as the European Union average, and almost three times higher than the national rate in the United States (http://www.suicide.org/index.html), totaling 32.2 suicides per 100,000 residents 
(Razvodovsky, 2008). Furthermore, Russia has the most hazardous patterns of drinking in the world (Rehm, Taylor \& Patra, 2006), with alcohol consumption in Russia being linked with suicide, especially for males (Razvodovsky, 2011), homicide (Chervyakov, Shkolnikov, Priedome \& McKee, 2002; Pridemore, 2002, 2006), and other violent deaths (Razvodovsky, 2010). All of these social issues contribute to the sense of instability and threat in the country, and signal the break of the social order. Similarly, economic hardships create a sense of financial insecurity and lack of resources. Taken together, these factors contribute to the development of the neighborhood disorder in Russia, particularly in the large cities such as Moscow, where the population is much more dense.

\section{Personal Control}

Personal control is the belief that one's life events and circumstances result from one's own choices, efforts and actions (Ross, 2011). The construct of personal control has been operationalized in different ways and appears in the literature under various names. Some of the most popular terms have been mastery (Pearlin et al., 1981), locus of control (Rotter, 1966), personal control (Mirowsky \& Ross, 1991) and, on the other end of the continuum, powerlessness (Seeman, 1959) and fatalism (Wheaton, 1980). All of these constructs overlap in their meaning and definition, and are frequently used interchangeably by researchers. For instance, Pearlin and colleagues (1981) defined mastery as "the extent to which people see themselves as being in control of the forces that importantly affect their lives" (p. 340), while Rotter (1990) defined locus of control as "the degree to which persons expect that a reinforcement or an outcome of their behavior is contingent on their own behavior or personal characteristics versus the degree to which persons expect that the reinforcement or outcome is a function of chance, luck, 
or fate, is under the control of powerful others, or is simply unpredictable" (p. 489). Furthermore, Seeman (1959) considered powerlessness and external locus of control to be related concepts. Similarly, Mirowsky and Ross (1991), who rooted their construct of personal control from Rotter's locus of control scale, frequently use terms "personal control" and "powerlessness" interchangeably in their writing. Since this study focuses on the relationship between neighborhood disorder and generalized trust, personal control and psychological well-being, which was outlined by Mirowsky and Ross (1991), the terminology that was used by Mirowsky and Ross will be used throughout this study. Beliefs about one's personal control are affected by one's lived experiences and social context, such as the neighborhood or the country where one resides. Research shows that individuals living in stressful and highly disordered neighborhoods experience a low sense of personal control (e.g., Geis \& Ross, 1998; Kim \& Conley, 2011; Ross \& Mirowsky, 2009). Stressful life events, in particular those that are negative and uncontrollable such as crime and danger in the neighborhood, can lower one's belief in their ability to influence their life circumstances (Mirowsky \& Ross 2003; Ross \& Mirowsky, 2003). Neighborhoods that have a high level of disorder, such as noise, vandalism, litter and crime indicate to its residents that social control is weak, that others living nearby are not concerned with maintaining order, and that those who have such power have abandoned them. This signals a high potential for harm, and creates a sense that life is chaotic and full of uncontrollable threats. In addition, most residents of disadvantaged neighborhoods do not have the ability to move to a better area, which adds to the lowered sense of personal control. For instance, Ross, Reynolds and Geis (2000) found that neighborhood stability (i.e., low residential turnover), although traditionally 
associated with positive outcomes, in fact is associated with higher distress for residents of disadvantaged areas. Disordered neighborhoods are frequently stable in residential turnover because residents believe they have no other options and not because they choose to stay in the same area. They feel trapped by their circumstances, and believe that they have no choice but to stay in their current location. The stress of living in a dirty, noisy and dangerous neighborhood with no opportunity for escape takes its toll on the residents in feelings of depression and anxiety. Perhaps it is the lowered sense of personal control that keeps residents trapped in their neighborhood, and they no longer try to escape the conditions in which they live.

A perceived sense of control is one of the cognitive bases of mental health and is essential for psychological and physical well-being (e.g., Mirowsky \& Ross, 1990, 2003). Extensive research shows a clear connection between a low sense of personal control and decreased psychological and physical health. For instance, Pudrovska and colleagues (2005) found that older adults with low sense of mastery over their lives, who experience economic hardship, were more depressed and anxious, and exhibited more physical symptoms, such as headaches, upset stomach, fatigue, chest pains and muscle aches, than those with high sense of mastery. Moreover, a different study found that higher perceived mastery was related to better health regardless of income (Lachman \& Weaver, 1998). Similarly, Ward (2013) found evidence for the inverse relationship between sense of personal mastery and self-rated global health and positive affect. Furthermore, external locus of control predicted higher mortality when combined with negative life events (Dalgard \& Haheim, 1998), and data from the national samples of older adults 
demonstrated that a sense of powerlessness is significantly related to health problems later in life and, ultimately, earlier mortality (Seeman \& Lewis, 1995).

It may be that the relationship between sense of personal control and health is bidirectional. That is, poor psychological and physical health may also lead to lowered sense of personal control. Or perhaps other variables may come into play and affect both the sense of personal control and health. For instance, Mirowsky and Ross (2003) suggested that social support may increase the sense of personal control, even in the presence of the stressful circumstances. It may be that one form of support may come from one's sense of spirituality. For example, Piedmont (1999) found that ST, particularly the Prayer Fulfillment and Connectedness facets, predicted one's perceived social support over and above personality factors, which supports this hypothesis.

Few studies have explored the relationship between personal control and one's personality. Existing studies have found a relationship between a sense of control and one particular factor of the FFM, Neuroticism, with a sense of control being negatively related to anxiety (Clarke, 2004; Joe, 1971), one of the primary components of neuroticism (Judge, Erez, Bono, \& Thoresen, 2002). With regard to the relationship between stressful environment and one's personality, Judge and colleagues (2002) found that neuroticism uniquely predicted one's level of stress and strain. That is, the higher an individual scores on the Neuroticism personality factor, the more likely they are to experience stress. Those scoring high on the Neuroticism factor also tend to use ineffective coping strategies, such as hostility, self-blame, withdrawal and passivity (McCrae \& Costa, 1986), which likely enhance the experience of stress in the first place. Therefore, individuals high in Neuroticism may be particularly affected by the 
neighborhood disorder, and consequently may particularly benefit from psychological buffers such as ST.

As discussed above, Russians are exposed to many stressors, and many geographic areas in Russia may be described in terms of the neighborhood disorder. Therefore, Russians may suffer from a low sense of personal control as a result of disordered environment and loss of sense of social order. This study aims to determine whether ST adds to the variance in personal control, as well as in several other psychosocial variables (i.e., generalized trust and psychological well-being), and whether it can offer any unique predictive validity over and above personality traits, such as Neuroticism. In sum, it is of interest whether individuals with higher scores on the STS are less affected by the neighborhood disorder and experience a higher sense of personal control even in highly stressful environments.

\section{Generalized Trust}

In addition to a lowered sense of personal control, another negative outcome of living amidst the neighborhood disorder is a lowered generalized trust in residents. Generalized trust is the notion that other people with whom we have no prior knowledge can be trusted (Dinesen, 2012). Generalized trust can be viewed as a continuum, rather than a dichotomous category, while mistrust is a belief that it is not safe to trust others, as they may pursue their own goals and may often harm, exploit or victimize you (Ross, Mirowsky \& Pribesh, 2001). The primary cognitive component of mistrust is suspicion of others, as well as consistent interpretation of others' actions and intentions as unsupportive, self-seeking and dishonest (Ross, 2011). Trust is important because it affects one's ability to form intimate relationships with others and, more broadly, achieve 
public order effectively by cooperating with other members of the community (Ross, Mirowsky \& Pribesh, 2002). Lack of generalized trust may also lead to mental health issues such as anxiety and depression. For instance, Ross and Mirowsky (2009) found that mistrust had a high and consistent positive association with psychological distress. It was found to directly increase anger and anxiety, because mistrusting individuals tend to expect the worst from others. In addition, individuals scoring low on trust were regarded as more depressed due to higher levels of alienation and fewer social ties that may offer solace.

In their first study exploring the construct of trust as it relates to powerlessness and neighborhood disorder Ross, Mirowsky and Pribesh (2001) found that mistrust increases with the prevalence of economic and social disadvantages in one's area of residence, and that this relationship in part is mediated by criminal victimization. Mistrust is a result of both individual characteristics and geographic location. Yet certain areas, especially those high in neighborhood disorder, may gather those who are more susceptible to mistrust and intensify their susceptibility through harsh circumstances and constant perceived threat (Ross, Mirowsky \& Pribesh, 2001). Individuals who feel powerless due to scarce resources and constant feelings of threat develop suspicion toward others. This phenomenon can be explained by the need to compete for scarce resources. If mistrust is the assumption that others are mainly looking out for themselves, then this assumption is correct in areas with no equitable distribution of resources, where the fittest individuals survive by obtaining the largest amount of capitals. In such areas suspicion is expected, and the consequences of misplaced trust may be devastating. Those with few resources cannot afford to lose much, and do not have the resources to make up 
for their losses. Therefore mistrusting others is a rational defense strategy against crime and victimization in areas where such risk is perceived to be high.

One theoretical amplifier of mistrust is a sense of powerlessness. The relationship between neighborhood disorder and mistrust is moderated by sense of personal control (Ross, Mirowsky \& Pribesh, 2001). Those who have a strong sense of personal control are less affected by negative life circumstances and show lower levels of mistrust (Ross, 2011). If an individual were able to maintain a high sense of control, he or she would also likely be more trusting towards others. Yet, a disordered environment lowers one's sense of personal control, resulting in a vicious cycle; living in a disordered neighborhood increases powerlessness, and consequently lowers one's trust in others. This phenomenon is known as structural amplification (Ross, Mirowsky \& Pribesh, 2001), and occurs when external conditions undermine the personal attributes of an individual that otherwise would have helped buffer the effects of the environment. In this case, a sense of powerlessness amplifies the relationship between neighborhood disorder and trust, while high sense of control and trust would have buffered an individual against negative influences on their physical and psychological well being. However, a sense of personal control and trust do not exist outside of an individual's social conditions. Thus, when these conditions are negative (i.e., neighborhood disorder), a sense of powerlessness develops, which increases the mistrust. Consequently, both negatively affect physical and mental health of an individual living under such conditions.

This study aims to investigate whether ST can help buffer the effects of the neighborhood disorder on one's sense of trust. Different facets of ST may enhance a sense of trust, consequently influencing well-being and decreasing the negative effects of 
a neighborhood disorder. Perhaps feeling as part of a whole humanity (i.e.,

Connectedness facet) can help an individual believe he or she and others are all working towards the same goals, and may decrease their susceptibility to mistrust towards others. Similarly, Universality, or belief in the unity and purpose of life, may enhance one's sense of personal control and trust in others, due to feelings of unity and closeness with other members of the community. Similarly, if one has a specific purpose in life, others would not be expected to bring harm unless that was one's life purpose. This notion generates a certain sense of control over life events, as life is not seen so chaotic and haphazard. Finally, receiving a sense of fulfillment from prayer (i.e., Prayer Fulfillment facet) may enhance a sense of personal control, since a praying individual believes he or she has done something to improve their circumstances by addressing the higher almighty power and feeling heard by it, which in turn may help increase a sense of generalized trust in ultimate fairness.

Another focus of this study is to determine whether personality traits add to the variance in generalized trust. Although neighborhood disorder tends to lower a sense of trust in its residents, personal characteristics also influence how susceptible an individual is to the effects of a disordered environment. For instance, there is a strong connection between one's sense of trust towards others and one's own prosocial tendencies, such as being honest and trustworthy (Rotter, 1980), or participating in voluntary associations (Brehm \& Rahn, 1997). Prosocial attitudes are evidenced in individuals scoring high on the Agreeableness personality factor. Trusting individuals are also more likely to reach out for social support when in need and accept any support that is given, which helps protect against victimization and mental health issues (Mirowsky \& Ross, 1983). These 
behaviors are common in individuals scoring high on the Extraversion personality factor.

Conversely, mistrusting individuals, such as those scoring high on the Neuroticism factor, tend to create and maintain conditions that seem to justify their beliefs by eliciting more hostility in others and rejecting any offered help. By doing so they become easy targets for crime and exploitation, which further diminishes their trust in others (Mirowsky \& Ross, 1983).

As previously discussed, Russians experience much social disorder in their immediate environment, suggesting the presence of the neighborhood disorder. Therefore, Russians may experience a lowered sense of generalized trust as a consequence of constant threat of victimization. This study seeks to understand whether ST and five personality factors can help explain the effects of the neighborhood disorder on a sense of generalized trust, as well as other outcomes (i.e., personal control and psychological well-being), and whether ST can offer any unique predictive validity over and above the personality traits, such as Agreeableness or Extraversion. That is, it is of interest whether individuals scoring higher on the STS are less affected by the neighborhood disorder and experience higher generalized trust even amidst stressful and disordered environments.

\section{Moderation Model}

It has been long known that living in a stressful environment negatively impacts one's mental health (Feldman, 2001; Pearlin et al., 1981; Ross \& Angel, 2005). Subjective alienation, a sense of separation from self and others, explains this connection between stressful and disordered environment (i.e., neighborhood disorder) and poor mental health. Two examples of such subjective alienation identified in the literature are 
a sense of personal control and generalized trust (Mirowsky \& Ross, 2003; Seeman, 1983). Previous research has established the mediating effect of a sense of personal control and generalized trust on the relationship between neighborhood disorder and psychological well-being (Mirowsky \& Ross, 2003; Ross \& Mirowsky, 2009).

One way to mitigate the harmful effects of the neighborhood disorder may be spiritually. Individuals scoring high on the STS may be better equipped to face negative life circumstances, since they may not feel alone in their struggles. These individuals may realize a larger meaning and purpose to their lives, which creates a will to continue living, and helps view negative circumstances as more purposeful and under somebody's control (for instance, individuals may explain their struggles as a test initiated by the higher power who is in ultimate control). Even under stressful circumstances, a belief in a higher power that ultimately has one's best interests in mind may offer a peace of mind.

Spirituality may impact all three outcome variables linked to the neighborhood disorder (i.e., sense of personal control, generalized trust and psychological well-being). For instance, high scores on STS may be associated with higher generalized trust. That is, believing that if one leads a good life and prays faithfully they will eventually be rewarded may increase a sense of trust that fairness will ultimately prevail. Similarly, feeling like there is unity and interconnectedness among all humans may positively influence a sense of trust, because there is a sense of shared responsibility and connection with others.

Additionally, individuals scoring high on the STS may evidence higher sense of personal control. That is, even if no other options are present, the ability to pray to a higher power, and faith that one's prayers will be heard, may help create a sense that one 
is doing something to improve their situation, and therefore one is at least in partial control over his or her environment. While Pargament, Koenig and Perez (2000) asserted that deferring control to God may be harmful to an individual's emotional well-being, other researchers have described psychological benefits of actively surrendering control to God (e.g., Wong-MacDonald \& Gorsuch, 2000). Furthermore, McElroy (1999) found that as long as God was perceived as loving, even passive or deferring coping strategies were effective when dealing with problems. Another study demonstrated a positive association between perceiving that God cares for one and ST (Greenway, Phelan, Turnbull, \& Milne, 2007), which suggests that a variety of religious coping strategies, such as deferring control to God or taking action by praying may be effective for individuals scoring high on the STS.

Finally, spirituality may also increase one's psychological well-being, because individuals may believe that they are not alone in facing their stressors, and that, ultimately, help is available from a powerful being. Social support has been linked to better psychological functioning and mental health (Cohen \& Wills, 1985; Kawachi \& Berkman, 2001), and spirituality can be a powerful source of support to many people. Previous research has already pointed to the positive relationship between spirituality and mental health (e.g., Greenway, Phelan, Turnbull \& Milne, 2007; Pargament, Smith, Koenig \& Perez, 1998; Pargament, Koenig \& Perez, 2000; Salsman et al., 2015), and this study seeks to expand on our understanding of the protective powers of spirituality in the face of stressful environment.

A theoretical framework used in this study, namely the negative relationship between the neighborhood disorder and psychological well-being, which is mediated by 
generalized trust and personal control, suggests that subjective alienation explains the link between stressful environment and mental health. Low ST may further influence this sense of subjective alienation, and therefore negatively impact all three outcome variables. All three facets of ST are related to a sense of connection with humanity and the spirits. For instance, an individual scoring low on the Connectedness facet may feel isolated and not as part of the larger group and humanity as a whole; a low score on the Universality facet may mean feeling like there is little unity and interconnection in life, and that there is no shared responsibility among creatures; and low score on the Prayer Fulfillment facet may translate into feeling little connection with spiritual powers. Thus, an individual scoring low on STS may feel more alienated from self and others, which will consequently negatively impact their mental health. Conversely, an individual scoring high on STS will be better protected from the negative influences of the neighborhood disorder, by buffering against the sense of subjective alienation and lowered psychological well-being.

Existing literature points to the buffering effects of spirituality on mental health. For instance, Reutter (2013) and Reutter \& Bigatti (2014) both found that spirituality moderates the relationship between perceived stress and psychological health. Other researchers found the moderating effects of spirituality on the relationship between chronic illness and psychological well-being (Ballew et al., 2012), traumatic life events and drug use (Staton-Tindall, Duvall, Stevens-Watkins, \& Oser, 2013), negative life events and suicidal ideation (Konick, 2009), interpersonal trauma and PTSD (Yedlin, 2013), and daily stress and depression (Elam, 2001). It is plausible to suggest that spirituality may act as a moderator within other theoretical frameworks, particularly those 
looking at mental health as an outcome, since the relationship between spirituality and psychological well-being is well established in the literature (Ano, \& Vasconcelles, 2005; Dein 2013; Greenway, Phelan, Turnbull \& Milne, 2007; Koenig 2012; Pargament, Smith, Koenig \& Perez, 1998; Pargament, Koenig \& Perez, 2000; Piedmont, 2001; Piedmont, 2007; Sandage and Jankowski, 2010).

This study aims to investigate the protective power of spirituality over one's psychological adjustment, and proposes that ST will moderate the relationship between stressful and disordered environment (i.e., neighborhood disorder) and psychosocial outcomes of trust, personal control and psychological well-being in Russians. It is expected that there will be a significant interaction effect, such that individuals scoring high on ST will show high levels of personal control, trust and psychological well-being in the presence of high levels of neighborhood disorder, while individuals scoring low on ST will show low levels of personal control, trust and psychological well-being in the presence of high levels of neighborhood disorder (Figure 1).

\section{Hypotheses}

H1: Spiritual Transcendence will form a sixth factor separate from the five personality factors in a Russian sample.

H2: Spiritual Transcendence will evidence incremental validity over and above the five personality factors in predicting psychological well-being, perceived control and generalized trust in a Russian sample.

H3: Spiritual Transcendence in a Russian sample will moderate the relationship between the neighborhood disorder and the outcomes of generalized trust, personal control and psychological well-being. 


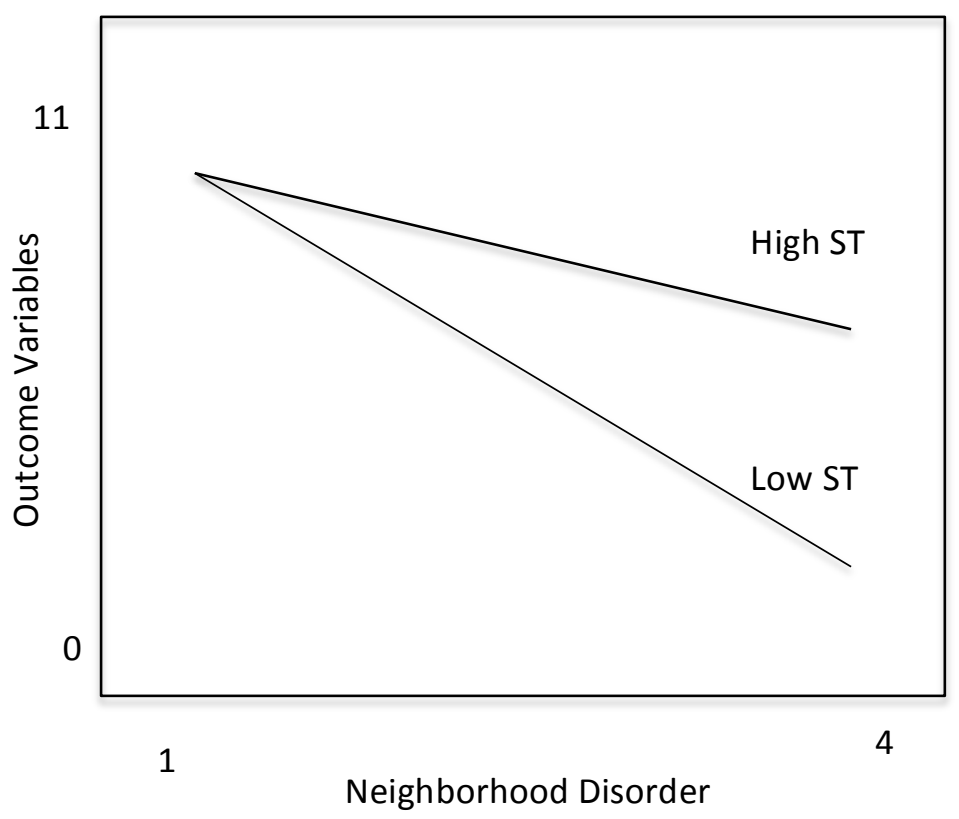

Figure 1. Expected interaction effect of the Spiritual Transcendence (depicted as ST) on the relationship between the neighborhood disorder and the three outcome variables of personal control, generalized trust and psychological well-being (depicted as Outcome Variables). It is expected that there will be a significant interaction effect, such that individuals scoring high on ST will show high levels on the outcome variables in the presence of high levels of the neighborhood disorder, while individuals scoring low on ST will show low levels on outcome variables in the presence of high levels of neighborhood disorder. There is no expected difference in the outcome variables for individuals scoring low on the neighborhood disorder. Such individuals are expected to score high on the three outcome variables regardless of their level of reported ST.

Though not hypothesized, a secondary wave of analysis will be conducted to examine individual effects of each facet of ST and FFM factor on generalized trust, personal control and psychological well-being. First, I expect to find that an individual who scores high on the STS will score high on measures of generalized trust, personal control, and psychological well-being because one's spirituality, in theory, helps buffer the negative effects of the neighborhood disorder. For instance, previous research found that Prayer Fulfillment and Universality facets of ST predict individual well-being and vulnerability to stress (Piedmont, 1999, 2007). Similarly, high scores on generalized trust 
may be associated with high scores on the Connectedness and Prayer Fulfillment facets, since these facets were found to predict prosocial behavior and perceived social support (Piedmont, 1999), characteristics that are related to generalized trust in individuals (Mirowsky \& Ross, 1983; Rotter, 1980). High scores on the sense of personal control may also be related to high scores on the Connectedness and Prayer Fulfillment facets, because these are related to perceived sense of social support, which is also related to higher sense of personal control (Mirowsky \& Ross, 2003).

Next, I will analyze the effects of the FFM on the three outcome variables. Specifically, I predict that (a) an individual scoring high on the Neuroticism factor will score low on generalized trust, personal control, and well-being, because these individuals tend to be anxious and low in resiliency. Therefore, they will be more affected by the neighborhood disorder, and experience negative outcomes evidenced in the literature (e.g., Ross \& Mirowsky, 2009). Conversely, (b) an individual scoring high on Agreeableness is expected to score high on trust, because these individuals tend to exhibit prosocial traits, such as honesty, forgiveness, generosity and cooperation. They tend to have more forgiving and trusting attitudes towards others and may be more willing to give somebody a second chance. Furthermore, (c) someone scoring high on Extraversion factor will show high psychological well-being. Extraverted individuals may have better social skills, because they are more experienced in the art of socializing and feel more comfortable being around others. They may seem more charismatic and attractive to others, due to higher levels of positive emotions and better acquired social skills, and therefore they might receive better treatment from those around them. They also likely have more social contacts and bigger social support network, which is related 
to psychological well-being (e.g., Emadpoor, Lavasani, \& Shahcheraghi, 2016; FullerIglesias, 2015; Weinberg, 2017). Similarly, (d) an individual scoring high on the Openness factor will evidence higher psychological well-being. These individuals tend to be more introspective, open-minded and cognitively flexible. They are more likely to approach and resolve new situations that arise, as opposed to avoiding them, which may ultimately lead to better outcomes (McCrae, 1996). For example, there is some evidence that high openness is related to better subjective well-being during difficult life transitions (Weiss, Freund, \& Wiese, 2012). Finally, (e) an individual scoring high on Conscientiousness is expected to score high on personal control, since such individuals tend to be more efficient, self-disciplined and deliberate in their actions, and likely feel higher sense of personal responsibility and control over their set goals and outcomes. 


\section{CHAPTER II: METHOD}

\section{Participants}

A total of 351 participants completed the consent process and started the survey. Of those participants 95 were removed from the data set due to large amounts of unanswered questions. Deletion criteria included leaving answers to at least one of the scales blank. Among the remaining data each scale was analyzed for each participant to ensure that missing data would not significantly impact the analysis of the results. No more than $15 \%$ of data per scale was missing for each of the remaining participants, which was deemed acceptable.

The remaining 256 participants comprised the final study sample. Among the final sample $21.1 \%$ identified as Christian, with an additional $44.9 \%$ specifying that they belong to Russian Orthodoxy subcategory of Christianity. Out of the remaining 34\% of the participants $24.6 \%$ identified as atheist, agnostic or not belonging to any particular religion, 2.7\% identified as Buddhist, 2\% identified as Muslim, 2.7\% wrote something else and $2 \%$ declined to answer. In terms of gender $28.9 \%$ of the sample identified as male. Participants' age ranged from 18 to 81 years old $($ Median $=33, \mathrm{SD}=12.1)$. Finally, participants were recruited from different geographical regions of Russia, with $65.6 \%$ participants residing in Western Russia (with $47.6 \%$ of the total sample residing in Moscow and 9.8\% in St. Petersburg), 10.5\% residing in Central Russia, 5.5\% residing in 
Southern Russia, 5.9\% residing in Eastern Russia (i.e., Siberia) and the remaining 12.5\% not specifying their location.

\section{Measures}

Demographic Questionnaire. Participants were asked to answer a brief demographic questionnaire asking for their age, gender, ethnicity, religious affiliation and in what geographic part of Russia they reside.

\section{The Assessment of Spirituality and Religious Sentiments (ASPIRES) scale:}

Short form. Developed by Piedmont (2004). The full ASPIRES scale assesses two dimensions: Religious Sentiments (RS) and Spiritual Transcendence (ST). It is appropriate for assessing individuals from a variety of faith traditions, as well as for use with nonreligious individuals. The full scale includes a 23 -item measure of ST that manifests a single factor comprising of three facets: Universality, a belief in the unity and the purpose of life (e.g., "All life is interconnected"; "I believe that there is a larger meaning to life"); Prayer Fulfillment, an experience of joy and content after a prayer or meditation (e.g., "I find inner strength and/or peace from my prayers or meditations"); and Connectedness, belief that one is part of the whole humanity and a sense of personal responsibility to others (e.g., "I am concerned about those who will come after me in life"; "I am a link in the chain of my family's heritage, a bridge between past and future"). The short STS version of the ASPIRES short form scale includes nine items, three from each of the facet scales. The chosen items were the ones evidencing the highest item-total correlation for their facet.

The second dimension of the full ASPIRES scale, RS, examines the value an individual attaches to his or her involvement in religious activities in a 12-item measure. 
This dimension is comprised of two facets; Religious Involvement, which examines how actively an individual is involved in religious activities and how important this activity is to him or her (e.g., "How often do you pray"; "How important to you are your religious beliefs"), and Religious Crisis, which assesses whether an individual experiences any conflicts or difficulties with God and/or faith community (e.g., feeling abandoned by God; feeling isolated from one's faith group). However, only the first four items from the Religious Involvement facet (dealing with frequency of engaging in various religious practices) are retained in the short form of the ASPIRES scale.

All 13 items of the ASPIRES scale short form are answered on a 5-point Likert scale, ranging from 1 (strongly agree) to 5 (strongly disagree). The measure was found to have adequate internal consistency for self-report version in the normative sample (alphas of $.89, .59$ and .68 for Prayer Fulfillment, Universality and Connectedness facets, respectively, with a total score of .76 for the STS; and alpha of .80 for Religious Involvement scales; Piedmont, 2012). Furthermore, each scale correlated significantly across self and observer ratings, indicating good convergent validity. The ASPIRES scale short form loaded on separate factors from the FFM, evidencing good discriminant validity from the five personality factors, and demonstrated good incremental validity by explaining additional variance in a number of psychosocial outcomes over and above the FFM (Piedmont, 2012).

In the current study, the ASPIRES measure showed adequate internal consistency (Cronbach's alphas of .88, .67 and .68 for Prayer Fulfillment, Universality and Connectedness facets, respectively, with a total score of .81 for the STS; and alpha of .55 for Religious Involvement items). While some alphas may appear to be on the lower end, 
it is important to remember that each facet is assessed with only three to four items, which likely affects the internal consistency of the scales. The Religious Involvement alpha was particularly low in the present study, lower than in the normative sample. One possible reason for that may be issues with translation of the items, which may have resulted in slightly changed meaning of some of the items and, consequently, lower internal consistency. Another explanation may be the specific background of the research participants in this particular study. Participants in this study were all nationals of former Soviet Union, where religion was strictly prohibited. As such, many Russians held onto their beliefs in private but avoided any public display of religiousness. This difference in public versus private engagement in religious activities may have influenced their responses to the Religious Involvement questions, which ask about both, public (e.g., "How often do you attend religious services?") and private (e.g., "How often do you pray?”) expressions of religion. Thus, in a Russian sample Religious Involvement may constitute two facets, internal and external. This separation may have lowered the internal consistency of the scale.

Big Five Inventory (BFI): Developed by John, Donahue and Kentle (1991), this 44-item scale was designed to measure the domains of the FFM. Each item consists of a short-phrase rated on a 5-point Likert scale ranging from 1 (disagree strongly) to 5 (agree strongly). Items are based on person-descriptive adjectives that were associated with each of the five factors of the FFM: Openness, Conscientiousness, Extraversion, Agreeableness and Neuroticism. The items were selected based on factor analyses and expert judgment to capture the core characteristics of each of the five factors of personality (John, Donahue \& Kentle, 1991). The measure includes 5 scales (one for each 
personality factor), each with eight to ten items. The BFI demonstrated good internal consistency (alphas range from .75 to .90 , with an average above .80; John \& Srivastava, 1999), high test-retest reliability (mean of .84) and good convergent validity with the NEO-PI-R ( $r=.78$; Rammstedt \& John, 2007). Furthermore, the BFI demonstrated strong discriminant validity (mean intercorrelation coefficient of .21 among scales), suggesting that the scales distinguish well among the five personality factors (Rammstedt \& John, 2007).

In the current study the Cronbach alphas for the five personality factors were as follows: .67 for Extraversion, .68 for Agreeableness, .77 for Conscientiousness, .81 for Neuroticism and .56 for Openness. Overall, these scales demonstrate adequate internal consistency, though alphas were lower than in the normative sample. Openness scale in particular showed lower reliability than that found in previous studies. Further analysis revealed that the Cronbach alpha for the Openness scale would improve to .67 if one item was dropped (item 41, "I see myself as someone who has few artistic interests"). However, the overall model fit was worse after this item was deleted, suggesting that it still adds meaningful variance to the results. A likely explanation for lower alphas for the scales is issues with the translation. Because the measure assesses personality traits using adjectives, any slight misinterpretation in the translation will have likely changed the intended meaning of the item, as it relates to its respective personality factor, thus lowering the internal consistency of the entire scale.

Mirowsky-Ross 2x2 Index. Developed by Mirowsky and Ross (1991), this 8item scale assesses one's sense of personal control. Items are balanced to reflect levels of control over both positive and negative circumstances on one's life (e.g., "The really 
good things that happen to me are mostly luck"; "I am responsible for my failures"). Responses are made on a 5-point Likert scale ranging from -2 (strongly disagree) to 2 (strongly agree) for items endorsing sense of control, and reverse coded items endorsing lack of control are included. The scale has been widely used in research on personal control (e.g., Cardarelli et al., 2007; Kim \& Conley, 2011; Ross \& Mirowsky, 2002; Ross, Mirowsky \& Pribesh, 2001; Schippe, 2012).

The Mirowsky-Ross Index is conceptually similar to other scales measuring some of the comparable constructs, such as Rotter's (1966) internal-external locus of control scale and Perlin et al.'s (1981) mastery scale. However, the Mirowsky-Ross Index has one principal difference: unlike other scales, it aims to eliminate defensiveness and response bias by balancing questions about good and bad outcomes (Mirowsky \& Ross, 1991). For instance, individuals who feel defensive claim different amounts of responsibility for good rather than bad outcomes. To control for this defensiveness, the Mirowsky-Ross Index has an equal number of bad and good outcomes listed within its items. Additionally, response bias may occur when all items of the measure have the same scale of responses, which may skew the overall responses for those who generally tend to agree or disagree with statements others make. Mirowsky and Ross (1991) controlled for this bias by asking an equal number of questions about internal and external sense of control. Their analysis indicated no significant variance from defensiveness on this measure, and no significant effects of agreement bias (Mirowsky \& Ross, 1991). Arguably, the Mirowsky-Ross Index constitutes a more valid measure of personal control, since it eliminates some of the major biases that frequently affect participants' responses. However, balancing the number of statements that claim versus 
deny a sense of control reduces the scale's internal consistency, since the items do not all follow the same direction. In their analysis of a student sample and a state-wide sample Mirowsky and Ross (1991) found alpha coefficients of .66 and .57, respectively. They argued that the reliability is lower due to small number of items, and provided calculations regarding how the reliability increases with the number of items. Later studies found slightly higher alpha coefficients of the scale. For instance, Schippe (2012) found alpha coefficients of .68 and .71 for two waves of analyses. Ross and Mirowsky (2009) also report an alpha coefficient of .71. In the current study the Cronbach alpha was .63, which is acceptable.

Ross-Mirowsky Neighborhood Disorder Scale. Developed by Ross and Mirowsky (1999), this 15-item scale assesses activities and conditions that residents of the neighborhood may perceive as sign of the breakdown of the social order. The scale measures physical signs of the disorder, such as graffiti, noise and vandalism in the neighborhood (e.g., "My neighborhood is noisy"), and social signs of the disorder, such as drug and alcohol use in the neighborhood, crime and trouble with the neighbors (e.g., "There is a lot of crime in my neighborhood"). The scale also includes reverse coded items to indicate absence of the disorder (e.g., "People in my neighborhood take good care of their houses and apartments"; "In my neighborhood, people watch out for each other"). The scale has two facets, order and disorder. All items are scored so that the higher mean score on the scale indicates a higher perception of the disorder. Disorder items are answered on a Likert scale ranging from 1 (strongly disagree) to 4 (strongly agree), and order items are reverse scored. The scale was found to have strong internal 
consistency, with an alpha coefficient of .92 (Ross \& Mirowsky, 1999). In the current study the Cronbach alpha was found to be .87 , which indicates good reliability.

Schwartz Outcome Scale-10 (SOS-10). Developed by Blais and colleagues (1999), the SOS-10 is a well-established 10-item measure of psychological health and well-being. It was initially designed to assess treatment outcomes for inpatient and outpatient clients (Blais et al., 1999). However, its strong psychometric properties and brevity have made it an attractive measure to use in research as well. Today, the SOS-10 is widely used by both researchers and clinicians (e.g., Dragomirecka, Lenderking, Motlova, Goppoldova, \& Šelepova, 2006; Haggerty, Blake, \& Siefert, 2010; Laux \& Ahern, 2003; Young, Waehler, Laux, McDaniel, \& Hilsenroth, 2003). Its items are answered on a 7-point Likert scale, ranging from 0 (never) to 6 (all of the time or nearly all of the time). The measure asks participants to rate the items based on how they have been feeling in the past 7 days (e.g., "I am able to have fun"; "My life is progressing according to my expectations"). It produces a total score, where higher scores are reflective of better psychological functioning and mental health. The SOS-10 has been found to have strong internal consistency (Cronbach alpha $=.96)$ and item-to-scale correlations ranging from .74 to .90 (Blais et al., 1999). Furthermore, the SOS-10 has demonstrated good construct validity, as it has been associated with measures of mental health (Blais et al., 1999; Haggerty, Blake, \& Siefert, 2010), depression and anxiety (Dragomirecka et al., 2006), substance use (Laux \& Ahern, 2003), maladjustment and distress in college students (Young et al., 2003) and the five-factor model (Haggerty, Blake, Naraine, Siefert, \& Blais, 2010). The current study evidenced good internal consistency of the scale (Cronbach's alpha of .87). 
Trust Scale. Developed by Reeskens and Hooghe (2008), this is a widely used 3item measure of generalized trust. This measure has been shown to be more reliable than the traditionally used 1-item measure (Reeskens \& Hooghe, 2008; Zmerli \& Newton, 2008). Also, according to Smith (1988), at least 3-item measures are necessary in crosscultural research in order to validly measure the construct (as cited in Reeskens \& Hooghe, 2008), since some concepts do not translate well into other languages. The trust scale consists of the following three questions: "Generally speaking would you say that most people can be trusted, or that you can't be too careful in dealing with people?"; "Do you think that most people would try to take advantage of you if they got the chance, or would they try to be fair?"; and "Would you say that most of the time people try to be helpful or that they are mostly looking out for themselves?" All questions are measured on an 11-point scale ranging from 0 (i.e., "You can't be too careful"; "Most people would try to take advantage of me"; "People mostly look out for themselves") to 10 (i.e., "Most people can be trusted"; "Most people would try to be fair"; "People mostly try to be helpful"). The items were found to be generally reliable in three immigrant groups in Northern Europe, with Cronbach's alphas ranging from .65 to .72 (Dinesen, 2012). The factor loadings for generalized trust were found to be equal across Europe (Reeskens \& Hooghe, 2008) and Asia (Tan \& Tambyah, 2011), suggesting the same latent structure of this construct across Eurasia. In the current study the Chornbach's alpha was .65, which is acceptable.

\section{Procedure}

Recruitment was conducted using the snowball sampling technique. The researcher contacted her friends and relatives in Russia with a request to complete the 
online questionnaire and to pass it along to their friends, family and co-workers, along with the same request to continue passing the questionnaire. The researcher also contacted her Russian acquaintances in the United States with a request to pass the questionnaire to their friends and relatives in Russia, in an attempt to obtain a sample from other parts of Russia, aside from Moscow, where all of the researcher's friends and family reside. A digital survey with a link to Qualtrics was distributed through e-mails and postings on Facebook and VKontakte (a Russian version of Facebook). After completion of the questionnaire, participants could choose to enter into a drawing for a chance to win one of three $\$ 40$ gift cards to Ozon.ru, a popular Russian online store. Study participants were adult Russian nationals who were born and raised in Russia. There were no other exclusion criteria. All recruitment materials and procedures were approved by the Institutional Review Board through the University of Louisville.

All items from the questionnaire were translated into Russian by the researcher, who is bilingual. Efforts were made to keep the same item content. The items were then back-translated into English by another bilingual individual, a Professor of Physics at the University of Missouri-Columbia. The original wording of the items was compared to the back-translated English version to ensure that the meaning of the item has not been lost in translation. The translation of the items that seemed to have changed its meaning after back-translation was re-evaluated and adjusted, by selecting different Russian words or phrases to better capture the meaning of the question as it was intended in the English version. A third bilingual individual, an internal medicine physician at Yale New Haven Hospital, back-translated the adjusted version of the translated items into English again. The comparison between the original wording and the second back-translation was 
satisfactory at this point. That is, the intended meaning of the questions appeared to be the same in the original and the translated English versions. 


\section{CHAPTER III: RESULTS}

\section{Preliminary Analyses}

The predictor variables for all analyses included the five personality domains of the FFM, overall ST score, and the neighborhood disorder scores. Outcome variables were psychological well-being, generalized trust and a sense of personal control. Prior to testing the hypotheses, the data was analyzed for missing responses. Additionally, preliminary analyses were conducted to test for violations of assumptions of multiple regression and CFA, the two analyses utilized to test the hypotheses in this study.

Missing Values. Listwise deletion approach was used to handle the missing data issue, and 95 cases were deleted due to missing significant amounts of data (i.e., leaving answers to the entire scale blank). Among the remaining 256 cases only $.45 \%$ of values were missing throughout the entire dataset. Furthermore, the Little's MCAR test revealed that values are missing completely at random $(p>.05)$. Therefore, missing values in the final sample did not present a significant issue.

Sample Size. In order to run a CFA analysis using Structural Equation Modeling (SEM) there needs to be an adequate sample size (Kline, 2011). According to Barrett (2007), a sample size of at least 200 is considered adequate. Furthermore, a power

analysis was performed, using G*Power 3.1 software (Faul, Erdfelder, Buchner, \& Lang, 2009) to determine an appropriate number of study participants. Using Cohen's (1988) 
criteria, it was determined that 103 participants would be needed to detect a medium effect size $(d=.50)$ with a power level of $80 \%$ and a criteria for statistical significance of $\alpha=.05$. Since the final sample in this study consisted of 256 participants, the sample size appears to be more than adequate.

Normality. The assumption of normality tests whether the observed data is normally distributed. This assumption needs to be met in order to conduct both a multiple regression and a CFA analyses. Normality was assessed by several criteria. First, the $5 \%$ Trimmed Mean was compared with the original mean, to determine whether the extreme scores had a strong influence on the mean. The comparison revealed that there is not much difference between the means for any of the variables (mean differences ranged from .001 to .03), indicating that the mean was not affected by the outliers for any of the variables. Next, skewness and kurtosis were examined for each variable. The values of skewness and kurtosis were below $|1|$ for all variables, with the exception of kurtosis for Personal Control, which was 1.032. This indicates that overall, data distributions approximate normal distribution, as there are no extreme deviations from zero in symmetry or peakedness of the distributions. Furthermore, the z-scores for skewness and kurtosis were below $|5|$ for all variables, also indicating that the distribution of data did not substantially deviate from normality. In addition, shapes of the histograms were examined, and were judged to approximate the bell-shape of the normal distribution as well. This was also supported by the inspection of the normal probability plots. For each variable observed data values formed a reasonably straight line, also suggesting a normal distribution. Finally, boxplots were examined. For all variables the whiskers of the boxplot were about equal in length and the median was in the center area of the boxplot, 
indicating normal distribution of scores. Furthermore, no extreme data points were detected. Some variables had several outliers, but the comparison of the mean to $5 \%$ Trimmed Mean indicated that these outliers did not substantially impact the mean. Overall, it was concluded that the data is roughly normally distributed and the assumption of normality is met.

Linearity. The assumption of linearity states that the relationship between independent and dependent variables is linear. To check this assumption residual plots were examined, which depict the error variance in the dependent variables that was not explained by the regression analysis (Meyers, Gamst \& Guarino, 2013). The scatterplots revealed data points randomly scattered around zero, as opposed to forming a distinct or curvilinear pattern, which indicates acceptable linear relationship among variables.

Homoscedasticity. The assumption of homoscedasticity states that the variance in data needs to be uniform; that is, levels of variance in psychological outcomes need to be constant across the five factors of personality and STS. This assumption was checked by examining the residual plots, which revealed data points randomly distributed, as opposed to forming a cone-shaped form, indicating a homoscedastic relationship between predictor and outcome variables.

Multicollinearity. The assumption of multicollinearity refers to checking the correlation among predictor variables (i.e., the neighborhood disorder, the ST and the five personality factors) to ensure that they are not too highly correlated, which would result in large portions of shared variance among predictors and small portions of unique variance. To check this assumption variance inflation factors (VIF) and Tolerance scores were examined. In order for the assumption to be met, the VIF value should be less than 
10, while the Tolerance score should be above .1. The analysis revealed that VIF scores ranged between 1.11 and 1.35, while Tolerance scores ranged between .74 and .90 , indicating absence of significant multicollinearity. Moreover, Pearson correlations between independent variables revealed small to medium relationships (Table 1), which further indicates absence of multicollinearity.

Outliers. The indicators of influence were examined to ensure that there are no deviant data points that affect the results. While boxplots revealed several data points that extended more than 1.5 standard deviations from the mean for most variables (with the exception of Agreeableness, Conscientiousness and Neuroticism) no extreme points (extending more than 3 standards deviations) were detected. Furthermore, no large differences between the $5 \%$ trimmed means and the original mean values were identified, indicating that the outliers did not have a strong influence on the mean for any of the examined variables. Finally, Cook's D and Leverage values for each variable were within the acceptable range, further indicating no significant influence of any deviant data points on the results. The data was also examined for multivariate outliers, which may be particularly influential when conducting a CFA analysis. The Mahalanobis Distance was computed for six predictors (i.e., ST and FFM) and for all participants p-values exceeded the .001 threshold (Tabachnick \& Fidell, 2007), suggesting that there are no multivariate outliers.

Correct Model Specification. In order to conduct a CFA analysis, the model needs to be properly specified, which means that each indicator variable is represented as having two sources of variability, a single factor that it is hypothesized to measure and all 


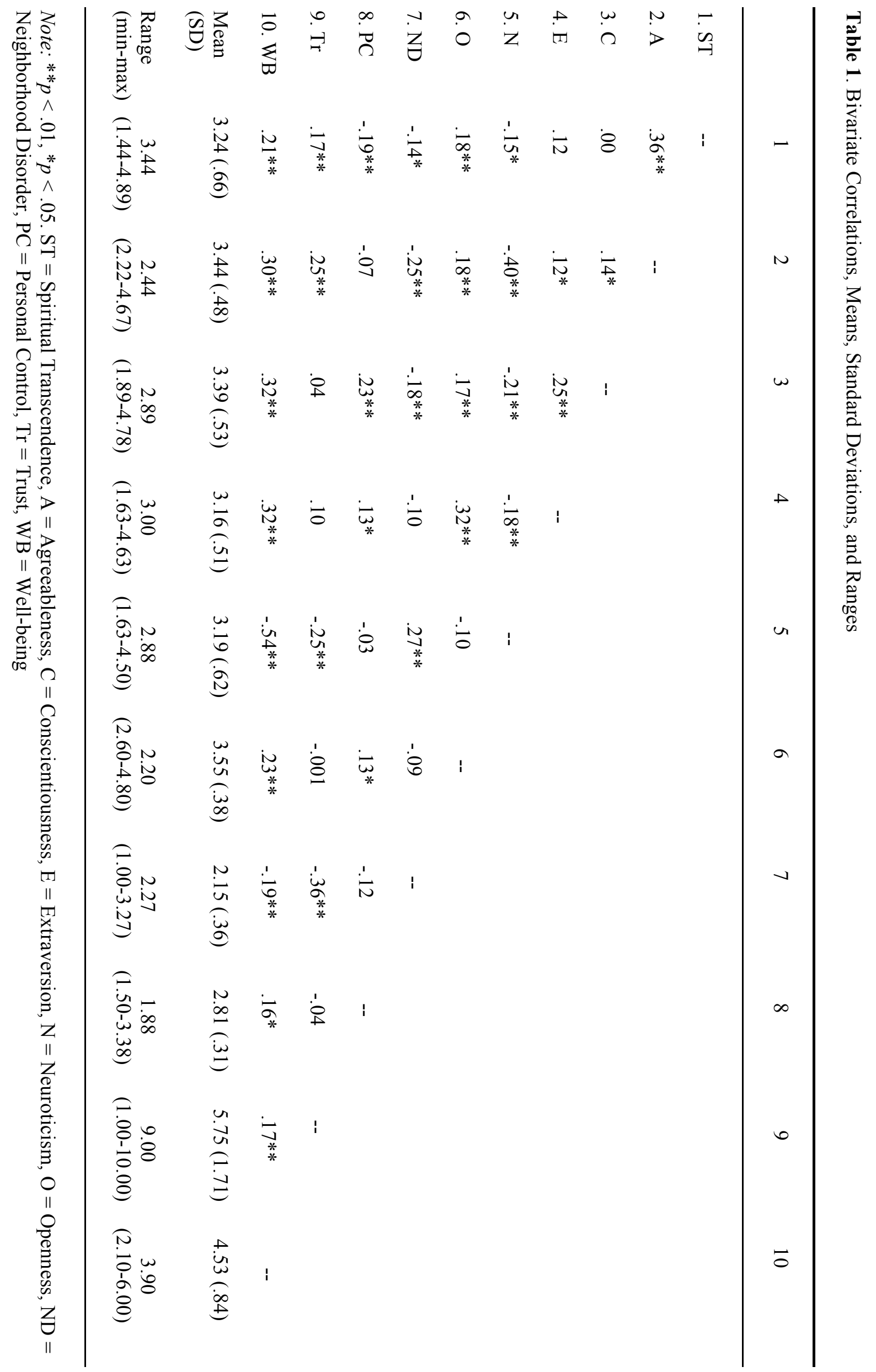


other unique sources of variance (represented by error variance; Klein, 2011). In addition, all variables must be assigned the role of either independent or dependent variables (Meyers, Gamst \& Guarino, 2013). The model (Figure 2) has six latent variables (i.e., ST, Openness, Conscientiousness, Extraversion, Agreeableness, Neuroticism) correlated with each other, with each factor represented by observed variables, and with error terms for each factor due to additional sources of variance unexplained by this model. That is, the ST has nine indicators for each item of the STS short form, and the five factors have eight to ten indicators each to reflect the items for each scale of the BFI. Therefore, all variables were assigned the role of either dependent or independent variable, with 59 independent variables (i.e., six factors and 53 error terms) and 53 dependent variables (i.e., 53 indicator variables).

\section{Primary Analyses}

Hypothesis 1. To test Hypothesis 1, that ST will form a sixth factor separate from the FFM, a Confirmatory Factor Analysis (CFA) was conducted. The responses to the BFI (i.e., the FFM measure) were expected to result in five factors, and the STS was expected to form a separate sixth factor, independent of the FFM, as established in previous research (e.g., Piedmont, 1999, 2001).

Prior to running the analysis, the model needed to be identified. In order to be identified, the model needs to have more known elements (i.e., observations) than the unknown (i.e., free parameters). In other words, degrees of freedom, which represent the difference between number of observations and number of model parameters need to have a positive value. If the degrees of freedom are negative, the model is said to be under-identified; if the degrees of freedom are equal to zero, the model is just-identified. 


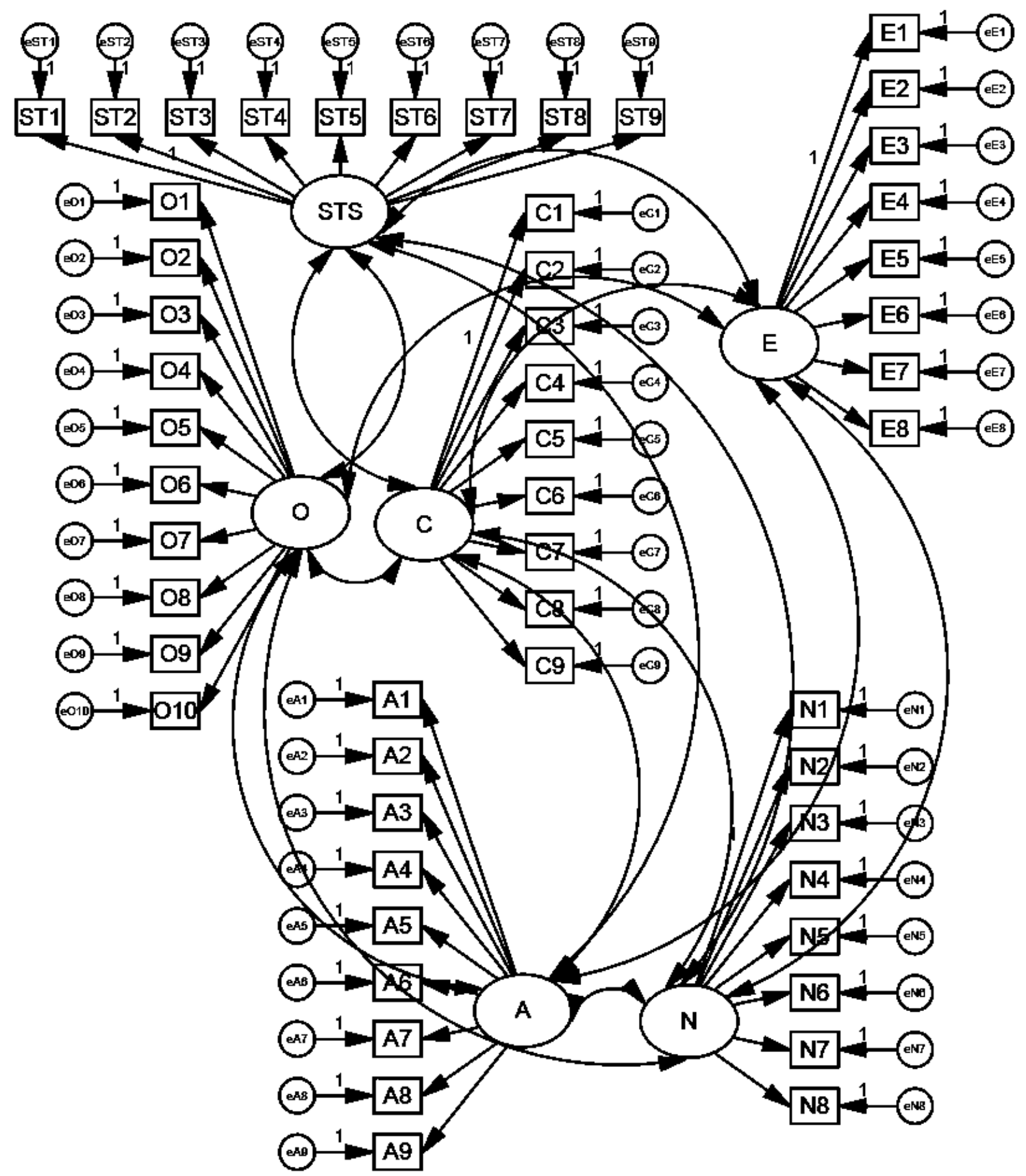

Figure 2. Confirmatory factor analysis model of the five factors of personality and Spiritual Transcendence. $S T S=$ Spiritual Transcendence, $E=$ Extraversion, $O=$ Openness, $C=$ Conscientiousness, $A=$ Agreeableness, $N=$ Neuroticism. 
First, model identification involves scaling of latent variables. Typically, this is done by assigning a value of 1.0 to the structure coefficient of one of the paths from each latent variable to one of the indicators that is most strongly correlated with the dimension represented by that latent variable, or to one of the indicators that has the best reliability (Meyers, Gamst \& Guarino, 2013). In addition, the paths leading from error terms to the indicators will be assigned a value of 1.0 as well. This is done to reduce the number of unknown parameters, so that the model is more likely to be identified.

To determine the number of known elements, the following formula is used (Meyers, Gamst \& Guarino, 2013):

$$
\text { Known elements }=[\mathrm{V}(\mathrm{V}+1)] / 2
$$

Where $\mathrm{V}$ represents the number of measured variables, which in this case is 53.

Therefore, the number of known elements is 1,431 . The number of free parameters, or the unknowns, is a sum of variances of latent factors, variances of error terms, and the unspecified coefficients relating latent variables to the indicators. In the present model the number of free parameters is 106 , which is less than the number of known elements. Therefore, the model is identified.

Once the model has been specified and identified, the proposed model was compared with the actual data gathered from the sample, to see whether the proposed model has a good fit with the sample. The model's adequacy was evaluated by examining the fit statistics, which consist of several values. First, the chi-square, which tests the relationship between the predicted and the observed relationships was examined. The chi square test was statistically significant $\left(\chi^{2}=2737.602, \mathrm{df}=1310, p<.001\right)$, but the chi square to degrees of freedom ratio was 2.09 , which is below the 2.5 threshold considered 
acceptable for a good model fit (Kline, 2011). Furthermore, according to Kenny (2015) the chi square for a sample size larger than 200 frequently shows statistical significance, because a larger power may pick up on trivial differences between the model and the data. This may be the case for the present model, given that the final sample size was larger than 200. Thus, other fit indexes were examined to supplement the chi-square statistic.

The root mean squared error of approximation (RMSEA) was .065, with $90 \%$ confidence interval of .062 to .069 , which indicates a good fit, as the values fall below the preferred .08 cutoff (Marsh \& Hau, 1996; Stevens, 2009). However, $p$ of Close Fit (PCLOSE) was statistically significant $(p<.001)$, suggesting that the model is worse fitting than the close-fitting model, and may contain specification error (Kenny, 2015). Furthermore, the Standardized Root Mean Square Residual (SRMR), which is an absolute measure of model fit, was .09 , which is below a .10 cutoff deemed acceptable (Cangur \& Ercan, 2015). Also, the Goodness of Fit Index (GFI) was .69, which is below recommended .90 cutoff (Bentler \& Bonett, 1980). Finally, the Comparative Fit Index (CFI) was .643 and Tucker-Lewis Index (TLI) was .625. Both values fall below the acceptable range of .90-.95 (Hu \& Bentler, 1999; Marsh, Hau \& Wen, 2004), which suggests that the model did not efficiently capture all the variance observed in data. Overall, the proposed model had an acceptable fit with obtained data set.

Table 2 specifies the modifications to the model and the resulting fit indices that were made based on theory and obtained modification indices. Overall, 23 modifications were made, consisting of correlating error terms for some of the items within each factor. For instance, items 6 ("Is reserved") and 21 ("Tends to be quiet") were correlated, 
because they belong to the same factor (i.e., Extraversion) and theoretically are similar enough to be potentially referring to the same concept. After 23 pairs of error terms were correlated, further suggested modifications were not made as they were deemed not theoretically sound.

After each modification, the chi-square difference test was performed between the two models to determine whether proposed modification will improve the model fit. Each modification resulted in not statistically significant chi-square difference, indicating that the more complex, modified model is preferred over the previous one. The resulting model had an improved fit. While chi-square was still statistically significant $\left(\chi^{2}=\right.$ $2190.384, \mathrm{df}=1287, p<.001$ ), this may have been due to a large sample size (Kenny, 2015). Furthermore, the chi square to degrees of freedom ratio became even less (1.70), indicating a good fit. The RMSEA of the final model was .052, with $90 \%$ confidence interval of .049 to .056 , which fall below .08 cutoff and suggests a good model fit. Furthermore, PCLOSE was not statistically significant $(p>.05)$, which also indicates a good model fit. Similarly, the SRMR for the final model was .08, which is below a .10 cutoff needed for an acceptable model fit. Yet, GFI was .76, which was still below the recommended cutoff. However, Sharma and colleagues (2005) argued that GFI is affected by the sample size and large number of indicators, and therefore caution is warranted when evaluating this statistic. Since the present study had 53 indicators, the GFI value may have been affected, and therefore it may not be an accurate evaluation of the model fit. Finally, the CFI and TLI values were higher for the final model (.774 and .758 , respectively), though they still fell below the acceptable range of .90-.95. Overall, the final model had a relatively good fit with the obtained data set. 
Table 2. Measurement Model Modifications

\begin{tabular}{|c|c|c|c|c|c|c|c|c|}
\hline Correlated terms & $x^{2}$ & $d f$ & $p$ & TLI & CFI & RMSEA & CI90 & PCLOSE \\
\hline Original Model & 2737.602 & 1310 & $<.001$ & .625 & .643 & .065 & $.062-.069$ & $<.001$ \\
\hline eST2 <-> eST3 & 2677.620 & 1309 & $<.001$ & .640 & .658 & .064 & $.061-.067$ & $<.001$ \\
\hline e1E <-> e36E & 2635.183 & 1308 & $<.001$ & .651 & .668 & .063 & $.060-.067$ & $<.001$ \\
\hline e6E <-> e21E & 2551.378 & 1307 & $<.001$ & .672 & .689 & .061 & $.058-.065$ & $<.001$ \\
\hline e19N <-> e39N & 2503.439 & 1306 & $<.001$ & .684 & .701 & .060 & $.056-.064$ & $<.001$ \\
\hline eST3 <-> eST5 & 2469.193 & 1305 & $<.001$ & .693 & .709 & .059 & $.056-.063$ & $<.001$ \\
\hline $\mathrm{e} 8 \mathrm{C}<->\mathrm{e} 18 \mathrm{C}$ & 2440.066 & 1304 & $<.001$ & .700 & .716 & .058 & $.055-.062$ & $<.001$ \\
\hline eST7 <-> eST9 & 2416.152 & 1303 & $<.001$ & .727 & .722 & .058 & $.054-.061$ & $<.001$ \\
\hline eST6 <-> eST9 & 2391.963 & 1302 & $<.001$ & .712 & .728 & .057 & $.054-.061$ & $<.01$ \\
\hline $\mathrm{e} 12 \mathrm{~A}<->\mathrm{e} 2 \mathrm{~A}$ & 2367.614 & 1301 & $<.001$ & .718 & .733 & .057 & $.053-.060$ & $<.01$ \\
\hline $\mathrm{e} 44 \mathrm{O}<->\mathrm{e} 30 \mathrm{O}$ & 2348.758 & 1300 & $<.001$ & .722 & .738 & .056 & $.053-.060$ & $<.01$ \\
\hline $\mathrm{e} 21 \mathrm{E}<->\mathrm{e} 31 \mathrm{E}$ & 2330.067 & 1299 & $<.001$ & .727 & .742 & .056 & $.052-.059$ & $<.01$ \\
\hline e6E <-> e31E & 2311.211 & 1298 & $<.001$ & .731 & .747 & .055 & $.052-.059$ & $<.01$ \\
\hline e $37 \mathrm{~A}<->$ e $2 \mathrm{~A}$ & 2298.525 & 1297 & $<.001$ & .734 & .750 & .055 & $.051-.059$ & $<.05$ \\
\hline e37A <-> e12A & 2275.933 & 1296 & $<.001$ & .740 & .755 & .054 & $.051-.058$ & $<.05$ \\
\hline eST1 <-> eST8 & 2252.325 & 1295 & $<.001$ & .745 & .761 & .054 & $.050-.058$ & $<.05$ \\
\hline eST2 <-> eST5 & 2237.040 & 1294 & $<.001$ & .749 & .764 & .053 & $.050-.057$ & $>.05$ \\
\hline $\mathrm{e} 1 \mathrm{E}<->\mathrm{e} 21 \mathrm{E}$ & 2228.784 & 1293 & $<.001$ & .751 & .766 & .053 & $.050-.057$ & $>.05$ \\
\hline $\mathrm{e} 28 \mathrm{C}<->\mathrm{e} 38 \mathrm{C}$ & 2221.133 & 1292 & $<.001$ & .752 & .768 & .053 & $.049-.057$ & $>.05$ \\
\hline e1E <-> e6E & 2212.465 & 1291 & $<.001$ & .754 & .770 & .053 & $.049-.057$ & $>.05$ \\
\hline e37A <-> e27A & 2206.615 & 1290 & $<.001$ & .755 & .771 & .053 & $.049-.057$ & $>.05$ \\
\hline e23C <-> e33C & 2200.459 & 1289 & $<.001$ & .757 & .772 & .053 & $.049-.056$ & $>.05$ \\
\hline $\mathrm{e} 7 \mathrm{~A}<->\mathrm{e} 42 \mathrm{~A}$ & 2194.889 & 1288 & $<.001$ & .758 & .773 & .053 & $.049-.056$ & $>.05$ \\
\hline $\mathrm{e} 44 \mathrm{O}<->\mathrm{e} 41 \mathrm{O}$ & 2190.384 & 1287 & $<.001$ & .758 & .774 & .052 & $.049-.056$ & $>.05$ \\
\hline
\end{tabular}

Note: Numbers indicate item numbers. $\mathrm{ST}=$ Spiritual Transcendence, $\mathrm{A}=$ Agreeableness, $\mathrm{C}=$ Conscientiousness, $\mathrm{E}=$ Extraversion, $\mathrm{N}=$ Neuroticism, $\mathrm{O}=$ Openness

Next, the maximum likelihood estimation procedure was performed in order to estimate the values of the parameters that would result in the best match between the data set and the proposed model (Meyers, Gamst \& Guarino, 2013). The path diagram displayed the standardized regression weights (i.e., factor loadings) for each indicator, summarized in Table 3. Some of the loadings did not reach the .40 threshold (Howard, 2016), which indicates that the items did not relate well with each other and did not load well on their proposed factor. 
For Spiritual Transcendence, three items representing the Connectedness facet (items ST2, ST3 and ST5) had the poorest fit, with factor loadings of .28, .20 and .17, and $\mathrm{R}^{2}$ of $.08, .04$ and .03 , respectively, which means that ST explains only $3 \%-8 \%$ of variance in these items. The three items representing Universality facet (items ST6, ST7 and ST9) were a better indicator of ST, with factor loadings of .59, .29 and .57, and $\mathrm{R}^{2}$ of $.35, .08$ and .33 , respectively. That is, ST explains $33-35 \%$ of variance in two items of the Universality facet (ST6 and ST9), while explaining only $8 \%$ of variance in item ST7 ("Although individual people may be difficult, I feel an emotional bond with all of the humanity"). Finally, items representing the Prayer Fulfillment facet (items ST1, ST4 and ST8) were the best indicators of ST, with factor loadings of .87, .84 and .92 and $\mathrm{R}^{2}$ of $.75, .70$ and .84 , respectively. That is, ST explains $70-84 \%$ of variance in Prayer Fulfillment facet. These results are consistent with previous findings, where Prayer Fulfillment was the most robust facet of ST, while Connectedness was the weakest one (Piedmont, 1999; 2001; Piedmont \& Leach, 2002).

The factor structure of the FFM appeared to be less robust than that established in previous research (e.g., Costa \& McCrae, 2002; McCrae, 2002). Items for Agreeableness, and Extraversion factors appeared to have particularly low loadings, with only 3-4 items from each factor reaching the .40 threshold (Howard, 2016). The Agreeableness factor explained $2-66 \%$ of variance in its items, with the lowest amount of variance explained in item 2 ("Tends to find fault with others", factor loading of .14) and the highest amount of variance explained in item 32 ("Is considerate and kind to almost everyone", factor loading of .81). The Extraversion factor accounted for $.5-65 \%$ of variance in its items, 
Table 3. Standardized Regression Weights

\begin{tabular}{|c|c|c|c|c|c|c|}
\hline Items & ST & A & E & $\mathrm{C}$ & $\mathrm{O}$ & $\mathrm{N}$ \\
\hline $\begin{array}{l}\text { ST1 } \\
\end{array}$ & .87 & & & & & \\
\hline ST2 & $.28^{* * *}$ & & & & & \\
\hline ST3 & $.20 * *$ & & & & & \\
\hline ST4 & $.84 * * *$ & & & & & \\
\hline ST5 & $.17 * *$ & & & & & \\
\hline ST6 & $.59 * * *$ & & & & & \\
\hline ST7 & $.29 * * *$ & & & & & \\
\hline ST8 & $.92 * * *$ & & & & & \\
\hline ST9 & $.57 * * *$ & & & & & \\
\hline BFI2 & & $.14 * * *$ & & & & \\
\hline BFI7 & & .52 & & & & \\
\hline BFI12 & & $.28^{*}$ & & & & \\
\hline BFI17 & & $.43^{* * *}$ & & & & \\
\hline BFI22 & & $.38^{* * *}$ & & & & \\
\hline BFI27 & & $.38 * * *$ & & & & \\
\hline BFI32 & & $.81^{* * * *}$ & & & & \\
\hline BFI37 & & $.38^{* * *}$ & & & & \\
\hline BFI42 & & $.44^{* * *}$ & & & & \\
\hline BFI1 & & & .31 & & & \\
\hline BFI6 & & & -.07 & & & \\
\hline BFI11 & & & $.81^{* * *}$ & & & \\
\hline BFI16 & & & $.74 * * *$ & & & \\
\hline BFI21 & & & .09 & & & \\
\hline BFI26 & & & $.20^{*}$ & & & \\
\hline BFI31 & & & $.18^{*}$ & & & \\
\hline BFI36 & & & $.46^{* * *}$ & & & \\
\hline BFI3 & & & & .73 & & \\
\hline BFI8 & & & & $.30 * * *$ & & \\
\hline BFI13 & & & & $.59 * * *$ & & \\
\hline BFI18 & & & & $.65^{* * *}$ & & \\
\hline BFI23 & & & & $.59 * * *$ & & \\
\hline BFI28 & & & & $.43 * * *$ & & \\
\hline BFI33 & & & & $.58 * * *$ & & \\
\hline BFI38 & & & & $.57^{* * *}$ & & \\
\hline BFI43 & & & & $.37^{* * *}$ & & \\
\hline BFI5 & & & & & $.68^{* * *}$ & \\
\hline BFI10 & & & & & $.53 * * *$ & \\
\hline BFI15 & & & & & $.64 * * *$ & \\
\hline
\end{tabular}


BFI20

Note: Values greater than $|.40|$ are given in bold. ${ }^{*} p<.05 * * p<.01, * * * p<.001$ $\mathrm{ST}=$ Spiritual Transcendence, $\mathrm{A}=$ Agreeableness, $\mathrm{C}=$ Conscientiousness, $\mathrm{E}=$ Extraversion, $\mathrm{N}=$ Neuroticism, $\mathrm{O}=$ Openness

with the least amount of variance explained in item 6 ("Is reserved", factor loading of .07 ) and the most variance explained in item 11 ("Is full of energy", factor loading of $.81)$.

Items for Openness, Conscientiousness and Neuroticism had higher loadings on their respective factors. The Openness factor had 5 out of 10 items reach the .40 threshold, with another 3 items being marginally acceptable (factor loadings ranging from .30 to .37$)$. Openness factor accounted for $4-46 \%$ of variance in its items, with the least amount of variance explained in item 30 ("Values artistic, aesthetic experiences", factor loading of .21) and the most variance explained in item 5 ("Is original, comes up with new ideas", factor loading of .68). For the Conscientiousness factor 7 out of 9 items reached the .40 factor loading threshold, with the other two items being marginally acceptable with factor loadings ranging from of .30 and .37 . The highest amount of 
variance that the Conscientiousness factor accounted for was 54\% in item 3 ("Does a thorough job", factor loading of .73) and the lowest variance accounted for was $9 \%$ in item 8 ("Can be somewhat careless", factor loading .30). For Neuroticism factor all items but one exceeded the .40 threshold, with factor loadings ranging from .51 to .75 , indicating that in the Russian sample this factor structure was the most robust out of the five personality factors. The one item that did not reach the .40 factor loading threshold was item 29 ("Can be moody", factor loading $.27, \mathrm{R}^{2}=.07$ ). The highest amount of variance that Neuroticism factor accounted for was 56\% in item 24 ("Is emotionally stable, not easily upset", factor loading of .75).

A post-hoc analysis was done, where items with low loadings (below .30) were deleted from the model. A chi square difference test revealed that the model fit was better after items with low loadings were deleted for Agreeableness (items 2 and 12), Extraversion (items 6, 21, 26 and 31) and Neuroticism (item 29) factors, but not for Openness (items 30 and 44). This may indicate some issues with these particular items, perhaps related to poor translation. Perhaps items for Openness factor did not have such translation issues, and therefore, even though the factor loading were low, the overall model fit was better with these items retained.

Next, correlations among the five personality factors and ST (Table 4) indicated that the relationship between ST and FFM is mostly weak and positive. Correlation coefficients ranged from .04 to .37 , with the weakest relationship being between ST and Conscientiousness factor, and comparatively strongest relationship being between ST and Agreeableness factor. The only negative correlation was that between ST and Neuroticism. Another model was estimated, where ST was not correlated with the five 
personality factors. The new model also had an acceptable fit $\left(\chi^{2}=2227.113, \mathrm{df}=1292, p\right.$ $<.001$ ), but the chi-square difference test was statistically significant, indicating that the original model, where all factors are correlated is a better fit. Therefore, the original model was retained. It is important to note though, that theoretically the six factors should not be correlated, as each represents it's own separate dimension. The model fit was likely better for the correlated model due to the sample specific error. That is, because the data is based on self-report, correlations among factors reflect similarities in responses made by the same participant, not the fundamental relationship among factors.

As for the relationship among the five personality factors, the correlation coefficients ranged from .20 to .61 , indicating weak to moderate relationships among factors. As expected, Neuroticism negatively correlated with the other four factors, while Agreeableness, Openness, Extraversion and Conscientiousness all correlated positively among each other. The strongest relationship was that between Openness and Extraversion, and the smallest correlation was between Neuroticism and Conscientiousness.

Table 4. Correlations Among Factors

\begin{tabular}{|c|c|c|c|c|c|}
\hline & 1 & 2 & 3 & 4 & 5 \\
\hline 1. ST & -- & & & & \\
\hline 2. A & $.37 * * *$ & -- & & & \\
\hline 3. $\mathrm{C}$ & .04 & $.22 * *$ & -- & & \\
\hline 4. E & $.23 * *$ & $.54 * * *$ & $.45 * * *$ & -- & \\
\hline 5. $\mathrm{N}$ & $-.19 *$ & $-.38 * * *$ & $-.20 *$ & $-.58 * * *$ & -- \\
\hline $6 . \mathrm{O}$ & $.25^{*}$ & $.27^{*}$ & $.36^{* *}$ & $.61 * *$ & $-.27 *$ \\
\hline & -- & & & & \\
\hline
\end{tabular}


Hypothesis 2. To test Hypothesis 2, which states that ST will demonstrate incremental validity over and above the FFM in predicting a sense of personal control, generalized trust and psychological well-being, a hierarchical multiple regression analysis was conducted. Three models were created, one for each outcome variable. For each regression model, the scores for the BFI (i.e., the FFM measure) were entered on the first step of the regression equation, with the ST scale scores entered on step 2. A partial F-test will determine whether the ST scale significantly increased predictiveness.

The results indicated that the FFM explained $38.9 \%$ of variance in psychological well-being $\left(R^{2}=.389, F[5,250]=31.805, p=.000\right), 8.1 \%$ of variance in personal control $\left(R^{2}=.081, F[5,250]=4.399, p=.001\right)$ and $10.1 \%$ of variance in generalized trust $\left(R^{2}=\right.$ $.101, F[5,250]=5.594, p=.000$ ), all of which are statistically significant. The ST explained 3.6\% additional variance in personal control $\left(R^{2}\right.$ change $=.036, F[1,249]=$ $10.292, p=.002), .8 \%$ of additional variance in psychological well-being $\left(R^{2}=.008, F[1\right.$, $249]=3.287, n s)$, and $.7 \%$ of additional variance in generalized trust $\left(R^{2}=.007, F[1\right.$, $249]=1.922, n s)$, both of which were not statistically significant. ST only offered $3.6 \%$ of additional explained variance in personal control over and above the FFM, and did not add statistically significant explanatory power to the outcomes of well-being and generalized trust. Therefore, Hypothesis 2 was only partially supported.

Hypothesis 3. It was anticipated that ST will moderate the relationship between neighborhood disorder and the outcomes of generalized trust, personal control, and psychological well-being (Figure 2). First, the interaction term between ST and neighborhood disorder was created, in order to test whether combined effect of these two predictor variables offers any additional statistically significant explanatory power to 
outcome variables over and above the main effects for STS and neighborhood disorder. Prior to creating the interaction term, the independent variables were first centered.

Three hierarchical regression models were created to test the moderation effect of ST on the relationship between the neighborhood disorder and the three outcome variables. For each model the data from the Neighborhood Disorder Scale was entered on first step, to account for its effect on the outcome variables, because previous research suggested a relationship between the neighborhood disorder and personal control, trust and well-being. The data from the STS was entered on step 2 of the model, to account for variance in outcomes that the ST explains on its own. Finally, the interaction term between neighborhood disorder and ST was entered on step 3 of the model, because it was hypothesized that the interaction will explain variance in the outcomes over and above each independent variable on its own. Personal control, trust and well- being were entered as the dependent variables for three regression models, respectively.

The results of the first hierarchical regression analysis showed that neighborhood disorder accounted for $1.1 \%$ of the variance in personal control, but it was not statistically significant $\left(R^{2}=.011, F[1,254]=3.83, \beta=-.12, \mathrm{t}[254]=-1.96, n s\right)$, while ST accounted for $4.6 \%$ of variance in personal control, which was statistically significant $\left(R^{2}=.046\right.$, $F[1,253]=12.35, \beta=-.22, \mathrm{t}[253]=-3.51, p=.001)$. Furthermore, the interaction effect of the neighborhood disorder and ST on personal control was not significant $\left(R^{2}=.011\right.$, $F[1,252]=2.90, \beta=.11, \mathrm{t}[252]=1.70, n s)$, suggesting no multiplicative effect between these two variables. The following equations summarize the results of the first analysis: 


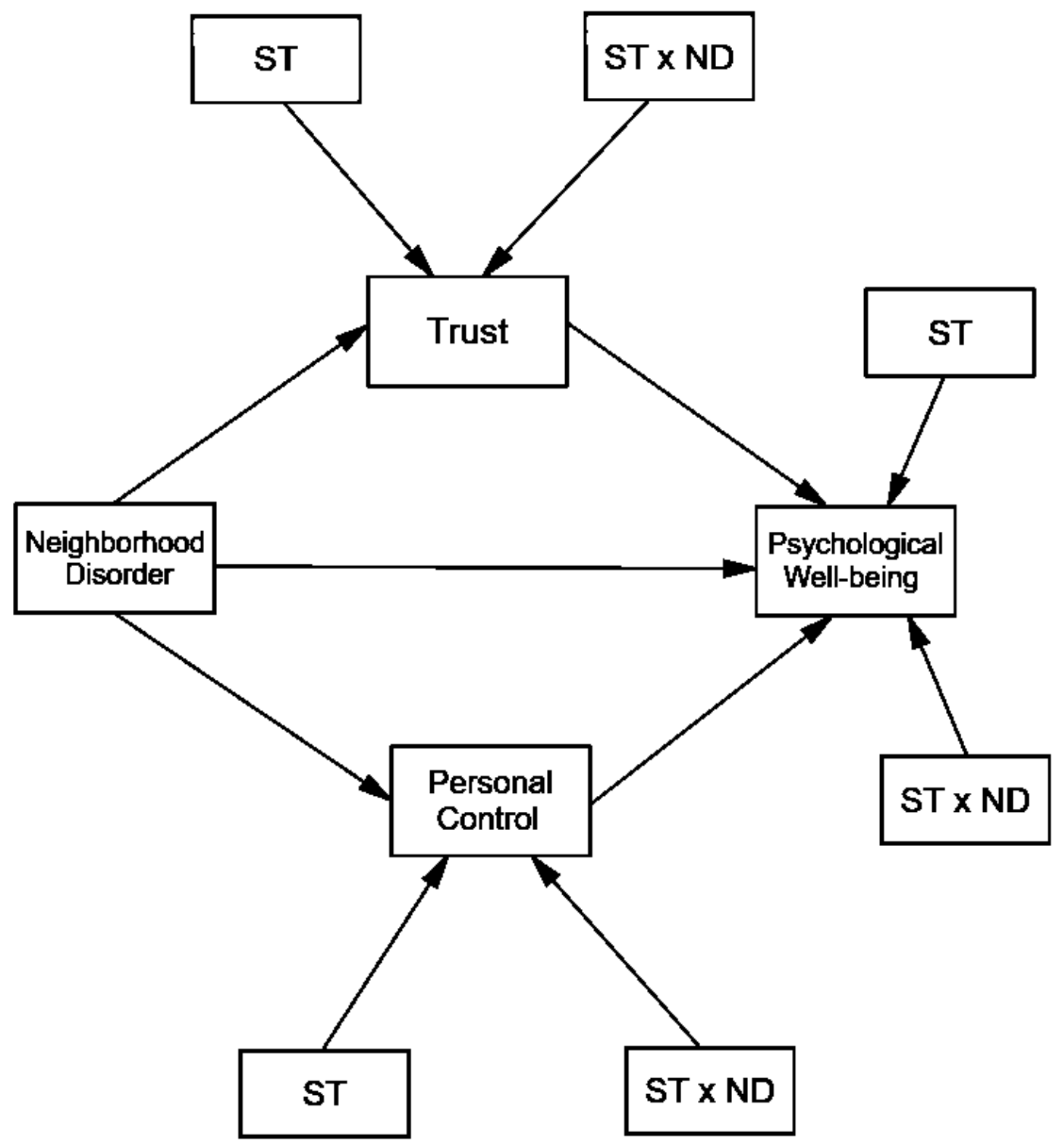

Figure 2. Moderation model testing the moderation effect of ST on the relationship between the neighborhood disorder and the three outcome variables (i.e., Trust, Personal Control, Psychological Well-being). $S T=$ Spiritual Transcendence. $S T x N D=$ interaction term. 
Model 1: $\mathrm{Y}(\mathrm{PC})=2.81-0.11(\mathrm{ND})$

Model 2: $\mathrm{Y}(\mathrm{PC})=2.81-0.13(\mathrm{ND})-0.10(\mathrm{ST})$

Model 3: $\mathrm{Y}(\mathrm{PC})=2.82-0.12(\mathrm{ND})-0.10(\mathrm{ST})+0.14(\mathrm{NDxST})$

The second hierarchical regression model, using Trust as the outcome variable, revealed that neighborhood disorder accounted for $12.8 \%$ of the variance in trust, which was statistically significant $\left(R^{2}=.128, F[1,254]=37.19, \beta=-.36, \mathrm{t}[254]=-6.10, p=\right.$ .000 ), while ST accounted for $1.4 \%$ of variance in trust, which was also statistically significant $\left(R^{2}=.014, F[1,253]=4.09, \beta=.12, \mathrm{t}[253]=2.02, p<.05\right)$. However, the interaction effect of the neighborhood disorder and STS on trust was not statistically significant, $\left(R^{2}=.003, F[1,252]=.98, \beta=-.06, \mathrm{t}[253]=-.99, n s\right)$, suggesting that $\mathrm{ST}$ did not moderate the relationship between neighborhood disorder and generalized trust. The following equations summarize the results of the second analysis:

Model 1: Y(Trust) $=5.74-1.69(\mathrm{ND})$

Model 2: $\mathrm{Y}($ Trust $)=5.74-1.61(\mathrm{ND})+0.31(\mathrm{ST})$

Model 3: $\mathrm{Y}($ Trust $)=5.73-1.66(\mathrm{ND})+0.30(\mathrm{ST})-0.41(\mathrm{NDxST})$

Finally, a third hierarchical regression model was conducted using psychological well-being as the outcome. The results of the analysis revealed that neighborhood disorder accounted for $3.8 \%$ of variance in well-being $\left(R^{2}=.038, F[1,254]=9.90, \beta=\right.$ $.19, \mathrm{t}[254]=-3.15, p<.01)$ and ST accounted for 3.3\% of variance in well-being $\left(R^{2}=\right.$ $.033, F[1,253]=9.08, \beta=.18, \mathrm{t}[253]=3.01, p<.01)$, both of which were statistically significant. However, the interaction effect of the neighborhood disorder and ST on wellbeing was also not statistically significant $\left(R^{2}=.007, F[1,252]=1.89, \beta=-.18, \mathrm{t}[252]=\right.$ $-2.92, n s)$. These results suggest that both neighborhood disorder and ST directly and 
significantly influence psychological well-being. However, ST does not moderate the relationship between neighborhood disorder and psychological well-being. The following equations summarize the results of the third analysis:

Model 1: $\mathrm{Y}(\mathrm{WB})=4.53-0.45(\mathrm{ND})$

Model 2: $\mathrm{Y}(\mathrm{WB})=4.52-0.39(\mathrm{ND})+0.23(\mathrm{ST})$

Model 3: $\mathrm{Y}(\mathrm{WB})=4.51-0.42(\mathrm{ND})+0.23(\mathrm{ST})-0.29(\mathrm{NDxST})$

Taken together, the results of the three regression analyses yielded no support for Hypothesis 3.

\section{Supplemental Analyses.}

Though not hypothesized, simple regression analyses were performed to examine individual effects of each facet of the ST (i.e., Prayer Fulfillment, Universality and Connectedness) on a sense of personal control, generalized trust and psychological wellbeing. That is, the three facets of ST were individually regressed on each of the three outcome variables, in a total of 9 separate simple regression models. The analyses revealed the following: for personal control, the effect of Connectedness $\left(R^{2}=.06, F[1\right.$, $254]=16.23, \beta=-.24, \mathrm{t}[254]=-4.0, p<.001)$ was significant and explained $6 \%$ of variance, while the effects of Prayer Fulfillment $\left(R^{2}=.01, F[1,254]=2.64, \beta=-.10\right.$. $\mathrm{t}[254]=1.62, n s)$ and Universality $\left(R^{2}=.01, F[1,254]=2.71, \beta=-.10, \mathrm{t}[254]=-1.65\right.$, $n s)$ were not statistically significant. For generalized trust, the effect of Universality $\left(R^{2}=\right.$ $.04, F[1,254]=16.23, \beta=.20, \mathrm{t}[254]=3.21, p<.01)$ was significant and explained $4 \%$ of variance, while the effects of Prayer Fulfillment $\left(R^{2}=.01, F[1,254]=1.93, \beta=.09\right.$. $\mathrm{t}[254]=1.39, n s)$ and Connectedness $\left(R^{2}=.01, F[1,254]=2.84, \beta=.11, \mathrm{t}[254]=1.69\right.$, $n s)$ were not statistically significant. Finally, for psychological well-being, both Prayer 
Fulfillment $\left(R^{2}=.06, F[1,254]=15.78, \beta=.24, \mathrm{t}[254]=3.97, p<.001\right)$ and Universality $\left(R^{2}=.03, F[1,254]=8.73, \beta=.18, \mathrm{t}[254]=2.95, p<.01\right)$ were significant predictors that explained $6 \%$ and $3 \%$ of variance, respectively, while the effect of Connectedness $\left(R^{2}=.00, F[1,254]=.59, \beta=.05, \mathrm{t}[254]=.77, n s\right)$ was not statistically significant.

A post-hoc analysis was performed to determine which facets and factors of ST and FFM were best predictors of the three outcome variables. All eight predictor variables (i.e., three facets of ST and five personality factors) were regressed on each of the three outcome variables in a series of three regression models, using forward entry. For personal control Connectedness facet of ST $\left(R^{2}=.06, F[1,254]=16.23, \beta=-.24\right.$, $\mathrm{t}[254]=-4.0, p<.001)$ was the best predictor, with Conscientiousness factor $\left(R^{2}=.05\right.$, $F[1,253]=13.48, \beta=.22, \mathrm{t}[253]=3.67, p<.001)$ next best predictor. For generalized trust Agreeableness $\left(R^{2}=.06, F[1,254]=17.56, \beta=.25, \mathrm{t}[254]=4.19, p<.001\right)$ was the best predictor, Neuroticism $\left(R^{2}=.03, F[1,253]=7.62, \beta=-.18, \mathrm{t}[253]=-2.76, p<.01\right)$ was the next best predictor and Universality facet of ST $\left(R^{2}=.01, F[1,252]=3.97, \beta=\right.$ $.13, \mathrm{t}[252]=1.99, p<.05)$ was the third best predictor. Finally, for psychological wellbeing Neuroticism $\left(R^{2}=.30, F[1,254]=106.82, \beta=-.54, \mathrm{t}[254]=-10.34, p<.001\right)$ was the best predictor, Extraversion $\left(R^{2}=.05, F[1,253]=20.90, \beta=.24, \mathrm{t}[253]=4.57, p<\right.$ $.001)$ was the next best predictor, Conscientiousness $\left(R^{2}=.03, F[1,252]=10.85, \beta=.17\right.$, $\mathrm{t}[252]=3.29, p<.01)$ was the third best predictor and Prayer Fulfillment $\left(R^{2}=.02, F[1\right.$, $251]=8.85, \beta=.15, \mathrm{t}[251]=2.97, p<.01)$ was the last best predictor. 


\section{CHAPTER IV: DISCUSSION}

Spirituality is often a source of solace and emotional support for individuals, especially as they face personal difficulties or are exposed to stressful environments. Previous studies found that ST influences a number of psychosocial outcomes over and above the five factors of personality and constitutes a sixth, separate factor (Piedmont, 1999, 2001, 2007; Piedmont \& Leach, 2002). The five personality factors are wellresearched constructs that have been extracted in multiple cultures and languages, and are linked to various psychosocial outcomes (e.g., Costa \& McCrae, 1992; McCrae \& Costa, 2003; McCrae, 2002; Rolland, 2002). The FFM provides context in which ST has been developed and studied.

The purpose of the current study was to investigate whether ST can be extracted as a sixth factor, separate from the five personality factors, and whether it has incremental validity in predicting a number of psychosocial outcomes (namely a sense of personal control, generalized trust and psychological well-being) over and above the five factors in a new, not previously studied culture and language (i.e., Russia). Another goal of the study was to investigate whether ST buffers against negative effects of stressful and disordered environment (i.e., neighborhood disorder) on the three outcome variables, which may provide evidence of its protective power.

Overall, the results of the current study evidenced the presence of ST in the Russian culture. The Prayer Fulfillment facet was the most robust, similar to findings in 
the U.S. and Indian cultures (Piedmont 1999, 2001; Piedmont \& Leach, 2002), while the Connectedness facet had the weakest loadings on the ST factor, which is found normatively. It is possible that Connectedness is not a very meaningful construct in Russian culture, or it may be that Connectedness is a more complex predictor than the other two facets, and its items do not overlap each other well. Similar findings were obtained in an Indian sample (Piedmont \& Leach, 2002), where Connectedness facet had the lowest factor loadings. Furthermore, in an Indian sample the model fit improved after the items representing Connectedness facet were deleted from the model, suggesting that the structure of ST held better without the Connectedness facet. However, in a Russian sample the model fit was worse after the Connectedness items were deleted, indicating that though Connectedness facet was the weakest one, it still captured valid variance. Thus, Russians may understand this construct differently than their U.S. counterparts, but the scale is still important in their culture.

Another explanation may be that these results reflect current psychological state of Russia's residents. Perhaps recent political events, such as the war with Ukraine, the resulting sanctions from Europe and deteriorating political relationship with the U.S. have left Russians feeling isolated and separated from the rest of the world. Future research may want to investigate this idea further, in order to determine whether politics, inherent Russian characteristics, the nature of the Connectedness facet itself, or something else may have resulted in poor structure of the Connectedness facet. It is important to note, however, that Connectedness facet has been weakly recovered in other international samples as well (Piedmont, 2007; Piedmont \& Leach, 2002), suggesting that these results may not be unique to Russian characteristics, but rather point to the weaker 
structure of the Connectedness facet itself. Yet, this scale was the strongest predictor of one of the outcome variables investigated in this study, a sense of personal control, pointing to its importance in the Russian culture despite its weak alpha.

In terms of the structure of the five personality factors in Russia, the Neuroticism factor had the highest factor loadings and the highest Cronbach's alpha, indicating highest internal consistency. The Conscientiousness factor also evidenced moderate to high factor loadings and high Cronbach's alpha, suggesting that items intended to represent this factor related well with each other. Conversely, items representing Agreeableness, Extraversion and Openness constructs did not load on their intended factors as well. Overall, the items did not fit together very well into one construct, and may have represented several separate constructs instead. This may be due to some uniqueness in Russian culture, though previous studies (e.g., Costa \& McCrae, 2002; McCrae, 2002) have extracted all five personality factors in Russia, with items loading on their factors as expected. Therefore, a more likely explanation is that there were issues with the translation of the items into Russian. Perhaps some nuances that these items were intended to pick up on were lost or misrepresented in a translated version.

The highest mean out of the five personality factors was that for Openness. This is similar to previous findings that revealed Openness as the most salient personality trait in Russians (Allik et al., 2009b; Terracciano et al., 2005). Interestingly, Allik and colleagues (2009b) suggested that this emphasis on openness may be a reflection of the spiritual nature of the Russian culture. They noted that it seems to be a cultural norm to endorse openness to non-materialistic values, which constitute a thread through Russian cultural history. In the current study Openness and ST had a significant positive correlation, 
though the correlation coefficient was small. It is important to keep in mind, though, that the Openness factor in this study had the lowest internal consistency, suggesting potential issues with it's measure, perhaps due to translation problems. Future research may wish to re-examine this relationship between Openness and spirituality in Russian culture using a more rigorous translation process for the measures utilized in the study.

In terms of the relationship between ST and the FFM, the results indicated that ST had mostly weak and positive correlation with the five personality factors. The strongest correlation was between ST and Agreeableness, suggesting that the more prosocial, honest and cooperative a person was, the higher he or she scored on the STS.

Extraversion and Openness were also positively correlated with ST, suggesting that openminded, curious, sociable and cheerful individuals reported higher levels of ST. Furthermore, the only negative correlation was that between Neuroticism and ST, indicating that the more prone to distressing emotions an individual was, the lower he or she scored on the STS. Finally, the relationship between Conscientiousness and ST was practically nonexistent, suggesting that one's spirituality and how efficient and selfdisciplined an individual is do not influence each other in a Russian culture.

It is important to note, though, that correlation coefficients were relatively low, falling in the range between .20 and .37 (with the exception of Conscientiousness factor, which was close to 0 ), indicating low to moderate correlations. This means that the relationship among these variables was not very strong to begin with, and variance in personality traits is not highly related to variance in ST. This supports the contention by the developer of the ASPIRES that ST forms a sixth personality factor (Piedmont 1999; 2001). Furthermore, the model fit was better with all six factors correlated than when ST 
factor was separated on its own, suggesting that there is a meaningful relationship among these variables. This further supports a theory that ST may represent a sixth factor of personality and operates in a similar manner as the FFM.

When interpreting the results of this study, it is important to keep in mind that while the model's fit was adequate, it was not as good as that reported in previous studies (Piedmont, 1999; Piedmont, 2001; Piedmont, 2007; Piedmont \& Leach, 2002). The fit of the model was improved after 23 error terms within all six factors were correlated, suggesting that responses to some of the items were not as correlated with one another as expected. The overall model fit may have been impacted by potential issues with the translation. It may be that some of the smaller variations between items were lost in translation, perhaps due to cultural and language differences. Future research may wish to more closely examine the potential differences in translation between the Russian and English versions of the measures.

One aim of this study was to test whether ST has incremental validity over and above the FFM in predicting the three outcome variables tested, namely psychological well-being, generalized trust and a sense of personal control. If ST were to evidence high incremental validity over the FFM, it would suggest that ST factor adds a strong and unique contribution to psychosocial outcomes after personality is accounted for and therefore may constitute a construct equitable to the FFM in its universality and influence on outcomes. As expected, the FFM significantly influenced all three outcome variables. However, the results yielded a small unique contribution of ST over and above the FFM to only one of the three outcome variables, a sense of personal control. ST did not explain 
any additional variance in generalized trust and psychological well-being after personality factors were accounted for.

A closer analysis revealed that only the Connectedness facet had a significant effect on a sense of personal control, a curious finding given that Connectedness was the least robust facet of ST in the Russian sample. Interestingly, the correlation between ST and personal control reveals a negative relationship. This finding means that the higher an individual scored on the STS, specifically on the Connectedness facet, the less sense of personal control he or she reported. This result seems to indicate that the more connected a person feels to the rest of humanity, spirits and his or her dead relatives or friends, the less in control over his or her own fate this individual feels. Perhaps an individual who feels a connection with the spirits and the deceased believes that they may offer guidance or support through some spiritual realms, and therefore relies on his or her own skills and wit less than someone who feels disconnected and alone, and does not expect any spiritual aid or guidance.

Surprisingly, ST did not add any additional explanatory power to psychological well-being after FFM was accounted for, though previous research (e.g., Piedmont, 2007; Piedmont \& Leach, 2002) found that such effect exists in other cultures. While ST significantly correlated with psychological well-being on its own, the effect seems to be lost once the five personality factors are accounted for. This indicates that in a Russian sample ST did not contribute to psychological well-being over and above the FFM, and variance in well-being is better explained by Russians' personality than their spirituality. Furthermore, out of the five personality factors Neuroticism contributed to psychological well-being the most, suggesting that whether or not an individual is prone to negative 
affect has the biggest impact on one's psychological well-being in Russia. This makes sense, as high scores on Neuroticism trait tend to correlate with high scores on depression and anxiety, a direct opposite of psychological well-being.

It is important to note, though, that these results were obtained when running a hierarchical regression. A supplemental analysis using forward entry revealed that ST significantly contributed to all three outcomes. That is, when both ST and the five personality factors were analyzed together, instead of controlling for all the personality variance before adding in the ST facets, the data indicated the unique and significant contribution of at least one facet of ST to each of the three outcome variables. This means that while ST may not predict psychological well-being and generalized trust over and above the FFM, it is still a significant predictor for these outcomes, indicating the importance of ST in the Russian culture.

Overall, this study suggests that ST does form a sixth factor, separate from the five personality factors, and it explains some variance in psychosocial outcomes. However, ST does not seem to offer much unique explanatory power to the outcome variables over and above the FFM, suggesting that spirituality may be closely intertwined with personality in Russian culture. It is important to note, however, that only three outcome variables were tested in this study. Previous research found that ST has incremental validity in explaining a number of psychosocial outcomes, such as prosocial behavior, happiness, life satisfaction, self-actualization, and vulnerability to stress (Piedmont, 1999, 2001, 2007; Piedmont \& Leach, 2002). Therefore, it may be that in Russian culture ST does not contribute unique variance to a sense of generalized trust and psychological well-being, but if other variables were tested, ST would reveal higher 
incremental validity. Future research may consider exploring a larger number of outcome variables and the relationship between ST, FFM and psychosocial outcomes.

Another purpose of this study was to investigate whether ST offers any protective power from the effects of stressful and disordered environment on psychological wellbeing, generalized trust and sense of personal control. The results indicated that ST did not act as a moderator between neighborhood disorder and the three outcome variables. While ST explained significant amount of variance in all three outcome variables, even after the effects of the neighborhood disorder were accounted for, it did not explain any significant variance in the outcome variables together with the neighborhood disorder, meaning that the relationship among neighborhood disorder and outcomes is not influenced by ST. In other words, ST does not become more salient in the presence of the neighborhood disorder. As a significant predictor of outcomes, ST acts as a protective factor on its own, not a specific protective factor from the effects of the neighborhood disorder.

Taken together, the findings of this study indicate the following: on its own, different facets of ST explain a significant amount of variance in all three outcome variables. Furthermore, when the effects of neighborhood disorder are accounted for, ST still adds significant explanatory power to all outcomes. However, once the five personality factors are taken into account, ST only adds a small additional explanatory power to personal control, but not to generalized trust or psychological well-being. Additionally, ST does not moderate the relationship between the neighborhood disorder and a sense of personal control, generalized trust and psychological well-being. It seems that out of the three outcome variables, ST has the biggest effect on a sense of personal 
control, over and above other explanatory variables, such as personality factors and the neighborhood disorder. However, in a Russian sample personality, particularly Neuroticism, is a better predictor of psychological well-being than ST, while the neighborhood disorder explains the largest amount of variance in generalized trust.

Though not the primary purpose of this study, the findings also extend the body of literature on the relationship among neighborhood disorder and trust, personal control and psychological well-being. Previous research has focused on the American culture (e.g., Ross, 2000; Ross, Mirowsky \& Pribesh, 2002). The current study demonstrates that the construct of the neighborhood disorder translates relatively well into another culture. Similar to findings of the previous studies (e.g., Mirowsky \& Ross, 2003; Ross, 2000; Ross \& Mirowsky, 2009; Ross, Mirowsky \& Pribesh, 2002), the neighborhood disorder significantly and negatively impacted a sense of generalized trust and psychological wellbeing in the Russian culture, demonstrating similar operation of the neighborhood disorder construct to the way it operates in the U.S. However, the neighborhood disorder did not significantly impact a sense of personal control in a Russian sample, a curious finding given that in the American culture the neighborhood disorder is significantly associated with a lowered sense of personal control (Ross, 2011).

One possible explanation for these results may be potential issues with the translation of the measures, similar to the differences in findings related to ST and FFM constructs from those in previous studies. However, because the relationship between the neighborhood disorder and the outcomes of generalized trust and well-being appeared similar to that established in previous research, it may be something about the Russian culture that resulted in not significant relationship between the neighborhood disorder 
and personal control. Personal control in this study was also negatively related to spirituality, which was an unexpected finding. Perhaps the construct of personal control operates differently in Russia. It may be, for example, that Russians utilize some unique coping strategies, not tested in this study, to manage the sense of powerlessness that has likely been widespread through the years following the collapse of the Soviet Union and ensuing government corruption, crime and lawlessness in the country. It may be that spirituality itself, particularly a sense of connectedness with others, has been a powerful coping mechanism that has carried Russians through the political and economic turmoil of the past century. Russians may have felt little personal control over their circumstances, with rapidly changing politics in the country, but they may have found a sense of control and empowerment in their communities. This may explain why neighborhood disorder in a Russian sample did not significantly influence personal control. While Connectedness facet did not have an interactive effect on outcomes together with the neighborhood disorder, it did have a direct effect on a sense of personal control. Thus, a sense of connection with others may help Russians not be impacted by their stressful and disordered environment.

\section{Limitations}

One of the biggest limitations of this study was that all scales were translated into Russian, and thus some important nuances of the measures may have been lost in the translation process. It may be that some concepts intended to be picked up on by the measures do not have a similar construct in the Russian language, or the concepts were not presented in as understandable manner as that found in US samples. This may also explain why the model fit of the six factors (i.e., FFM and ST) was not as good as that 
established in previous research. Future research may wish to incorporate a more rigorous translation process and examine the choice of terms used in the Russian translation more closely. It may also be beneficial to consult religious experts in Russia to better understand the appropriate choice of terms that could better capture the nature of spirituality in the Russian culture. Finally, while the developer of STS was consulted in the translation process of the ASPIRES scale, the personality researchers may need to be consulted when translating the FFM measure to ensure that the intended meaning of the scales is captured in the Russian translation.

Potential translation issues and cultural references are an inherent difficulty of cross-cultural research utilizing an etic study design. In this study measures developed in the context of one culture were applied to a different culture, and therefore what was deemed important in the original culture was imposed on the new one. For example, the construct of ST may not have captured all nuances of Russian spirituality, which might explain it's lack of strong association with the outcome variables over and above the FFM. Future research may wish to consult Russian religious and spiritual experts to better understand the nature of spirituality in Russia. An emic approach to studying spirituality in Russia may reveal more culture-specific constructs that better describe spiritual experiences of Russian nationals.

Another reason why the variables did not perform as expected may be the selection of the measures. For example, the current study used the ASPIRES short form to measure ST, which consists of 9 items, while previous studies utilized the full ST scale that has 23 items. It is more difficult to obtain a good model fit when fewer items represent a construct (Kline, 2011). Therefore, the model fit may have been affected by 
only 3 items representing each of the facets of ST. Perhaps if the original, longer scale was used, it would have provided more statistical power, resulting in a cleaner factor structure and stronger predictive coefficients.

Furthermore, a different measure was used to assess the FFM than those used in previous studies exploring ST and personality in Russia. While previous studies used NEO-PI-R (Costa \& McCrae, 1992; Piedmont, 2001) and Bipolar Adjective Rating Scale (BARS; McCrae \& Costa, 1985, 1987; Piedmont, 1999; Piedmont, 2007; Piedmont \& Leach, 2002), the current study used the Big Five Inventory (BFI), in an attempt to reduce the amount of time needed to complete the questionnaire. Though the BFI showed good convergent validity with NEO-PI-R (Rammstedt \& John, 2007), it is possible that conceptually there were some small differences, which is why results of previous studies were not replicated in this study. As noted with the STS above, the translation of the BFI utilized in this study may not have captured all the nuances of the measure as the English version. Therefore, caution is needed when comparing the results of this study with other studies investigating personality in Russia or the relationship between the FFM and ST. Because the BFI evidenced worse model fit than that in previous research, which was able to extract five personality factors in Russian samples, the results of this study suggest that a more rigorous translation process is necessary.

Finally, the snowball sampling technique was used, which may have resulted in unrepresentative sample. Participants were obtained through personal references from the author's friends and relatives, all of whom may have had some commonalities. For instance, majority of this author's friends and relatives have a college degree, and likely are acquainted with other well-educated individuals. Therefore, higher education or 
socio-economic status may have been overrepresented in this study. Such potential bias makes it difficult to make inferences about the entire Russian population.

\section{Implications for Counseling}

The current study has several implications for mental health professionals. Spirituality is rarely discussed in counseling sessions, though many clients report wanting to discuss it in therapy (Cashwell et al., 2013; Rose, Westefeld, \& Ansely, 2001; Weld \& Eriksen, 2007). The results of this study point to the importance of spirituality, especially as it relates to a sense of personal control. Interestingly, individuals who endorse high levels of spirituality, particularly feelings of connectedness with humanity and the deceased, demonstrate lower sense of personal control over their own lives. This may be an important connection to keep in mind, particularly when counseling Russian clients, as locus of control is related to mental health (Gore, Griffin, \& McNierney, 2016; Grob, Little, Wanner, Wearing, \& Euronet, 1996; Lachman \& Weaver, 1998). Thus, for clients who report external sense of control or a sense of powerlessness it may be particularly beneficial to discuss their spiritual beliefs. Perhaps if they better understood this connection between their spirituality and a lowered sense of personal control, they could feel more empowered or find better coping strategies.

Another important finding of this study is that the personality of the Russian nationals is the strongest predictor of their psychological well-being. While personality is difficult to change, it may be beneficial to discuss this connection in session in an attempt to raise insight. Sometimes simply understanding the underlying factors of one's troubles can bring relief. Out of the five personality factors Neuroticism was the strongest predictor of psychological well-being, suggesting that the extent to which an individual is 
prone to negative emotions directly impacts their mental health. Thus, spending time in session on learning some coping skills to manage neurotic personality traits may be beneficial when working with Russian nationals who demonstrate high neuroticism. Other four personality factors also had a significant impact on psychological well-being, however, which indicates that considering all aspects of a client's personality may be beneficial in treatment. For example, helping client become more open to new ideas and experiences, or working towards raising Agreeableness traits, such as forgiveness and cooperation, may help contribute to improved psychological well-being.

Finally, this study demonstrated that the construct of the neighborhood disorder operates somewhat similarly in Russia, as compared to its manifestation in the United States. That is, neighborhood disorder significantly and negatively affects psychological well-being and generalized trust in Russia. However, neighborhood disorder does not significantly impact sense of personal control, unlike its effect in the U.S. These are important findings to consider when working with Russian individuals affected by the neighborhood disorder. Counselors who understand this connection may be better equipped to work with such clients. Raising insight into how their immediate environment might be affecting their psychological well-being might be beneficial to some clients, who then might be able to find better coping strategies to counteract the effects of their disordered neighborhood. Understanding that the neighborhood disorder significantly and negatively impacts a sense of generalized trust might also be helpful in counseling. Perhaps working to increase a sense of trust in such clients might help mitigate the harmful effects of the neighborhood disorder on their psychological wellbeing, since previous research established that trust mediates the relationship between the 
neighborhood disorder and mental health (Mirowsky \& Ross, 2003; Ross \& Mirowsky, 2009).

The current study also points to the significant influences of personality and spirituality on the sense of generalized trust and psychological well-being. In particular, for a sense of generalized trust Agreeableness and Neuroticism were significant predictors, so focusing on these personality traits in counseling may be especially helpful. Similarly, Universality facet of ST was a significant predictor of trust, so discussing a client's sense of unity and interconnectedness among all humans might also be helpful when working with clients impacted by the neighborhood disorder. As for psychological well-being, all five personality factors were significant predictors, as well as Prayer Fulfillment and Universality facets of ST. Therefore, discussing aspects of their personality, their sense of unity with others and their spiritual practices might help clients find better coping strategies to manage the effects of their disordered environment.

\section{Future Directions}

The broad purpose of this study was to test the structure of ST in a new culture and language in an attempt to understand whether ST constitutes a universal construct and whether it impacts psychosocial outcomes. Overall, the findings of this study offer further support to the notion of the universality of ST, as it was extracted in yet another culture and a different language, and significantly impacted all psychosocial outcomes that were tested. However, ST did not evidence much significant impact on outcomes over and above the five personality factors, contrary to previous findings in other cultures and languages. This suggests either some uniqueness of the Russian culture, or some issues with the study design. 
Future research may consider a few changes when designing another study. First, as discussed previously, a more rigorous translation process is necessary in order to rule out infelicities in language. A validation study, comparing the responses to the original English version and the translated version of the measures will be beneficial first step. Second, different measures of ST and FFM may lead to a better model fit, so that more valid conclusions can be drawn about the constructs of personality and spirituality in Russian culture. Third, more outcome variables need to be tested in order to better understand whether ST offers much unique predictive power to outcomes over and above the personality factors, or if in the Russian culture spirituality is more blended with personality than in other countries previously tested. For example, does spirituality in Russia predict life satisfaction, moral values or social attitudes over and above personality?

Additionally, current study did not investigate the influence of the religious involvement measure on the outcomes. Future research may wish to include this dimension in the analyses in order to gain a more comprehensive assessment of how spirituality affects the outcome variables. It may be, for example, that in Russia religious involvement is a stronger predictor of some psychosocial outcomes than perhaps the five personality factors or ST. Or, on the contrary, religious involvement may be a weaker predictor of outcomes in Russia, as compared to some other cultures, since religious expression has been suppressed for decades during the Soviet Union regime. Either way, including religious involvement scale in the analyses will provide a better understanding of how spirituality operates in Russia. 
It may also be of interest to take a closer look at the construct of the neighborhood disorder and how it operates in Russia. For instance, current study did not investigate whether personal control and trust mediate the relationship between the neighborhood disorder and psychological well-being in Russia, an association that has been established in previous studies in the U.S. (Mirowsky \& Ross, 2003; Ross \& Mirowsky, 2009). Furthermore, the current study found that neighborhood disorder is not a significant predictor of personal control, a curious and unexpected finding that may need further investigating. Also, the present study did not examine the relationship between different personality traits and the psychological consequences of living in a highly distressing environment, though this study did find that personality factors are significant predictors of all three outcome variables. Does personality moderate the relationship between the neighborhood disorder and psychosocial outcomes? Do certain personality traits help protect from the negative effects of the neighborhood disorder?

Future research may also wish to continue exploring spirituality in Russia, to better understand its operating mechanisms. Perhaps employing an emic study design might reveal more unique intricacies of spiritual experiences of Russians. Despite over 70 years of Communist rule and suppression of all religious and spiritual expressions, the ST was still clearly recoverable in the Russian dataset and its facets significantly predicted all tested psychosocial outcomes, further supporting the hypothesis of the universality of the ST as an intrinsic source of human motivation. While quantitative study design is a useful step in understanding broad patterns and trends in a new culture, a qualitative design may provide richer and more detailed information about the nature of spirituality in Russia. An emic approach would be to focus on finding constructs within Russian 
culture that may better capture the spiritual experiences relevant to this particular culture than does the ST. While collecting data for this study, the author received feedback from family and friends who participated in the study, noting that they felt like questions targeted at capturing ST where not relevant to their lives and unrepresentative of what they understood to be spirituality. It would be interesting to explore what questions better reflect the enigmatic Russian soul and what variables feel more relevant to current experiences of Russian nationals. 


\section{REFERENCES}

Allik, J., Realo, A., Móttus, R., Pullmann, H., Trifonova, A., \& McCrae, R. R. (2009a). Personality traits of Russians from the observer's perspective. European Journal of Personality, 23(7), 567-588. doi:10.1002/per.721

Allik, J. J., Mõttus, R. R., Realo, A. A., Pullmann, H. H., Trifonova, A. A., McCrae, R. R., \& Meshcheryakov, B. G. (2009b). How a national character is constructed: Personality traits attributed to the typical Russian. Cultural-Historical Psychology, 1, 2-18.

Allik, J., Realo, A., Mõttus, R., Pullmann, H., Trifonova, A., McCrae, R. R., \& ... Korneeva, E. E. (2011). Personality profiles and the "Russian soul": Literary and scholarly views evaluated. Journal of Cross-Cultural Psychology, 42(3), 372-389. doi:10.1177/0022022110362751

Aneshensel, C. S. \& Sucoff, A. C. (1996). The neighborhood context of adolescent mental health. Journal of Health and Social Behavior 37, 293-310. doi: $10.2307 / 2137258$

Ano, G. G., \& Vasconcelles, E. B. (2005). Religious coping and psychological adjustment to stress: A meta-analysis. Journal of Clinical Psychology, 61, 461480. doi: 10.1002/jclp.20049

Ardichvili, A. (2006). Russian Orthodoxy worldview and adult learning in the workplace. Advances in Developing Human Resources, 8(3), 373-381. doi:10.1177/1523422306288430

Aro, A.R., Nyberg, N., Absetz, P., Henriksson, M., \& Lonnqvist, J. (2001). Depressive symptoms in middle-aged women are more strongly associated with physical 
health and social support than with socioeconomic factors. Nord J. Psychiatry, 55, 191-198. doi: 10.1080/08039480152036074

Bailis, D. S., Segall, A., Mahon, M. J., Chipperfield, J. G., \& Dunn, E. M. (2001). Perceived control in relation to socioeconomic and behavioral resources for health. Social Science \& Medicine, 52(11), 1661-1676. doi:10.1016/S02779536(00)00280-X

Ballew, S. H., Hannum, S. M., Gaines, J. M., Marx, K. A., \& Parrish, J. M. (2012) The role of spiritual experiences and activities in the relationship between chronic illness and psychological well-being. Journal of Religion and Health, 51(4), 2012, 1386-1396. doi: 10.1007/s10943-011-9498-0

Barrett, P. (2007), "Structural Equation Modelling: Adjudging Model Fit," Personality and Individual Differences, 42 (5), 815-24. doi: 10.1016/j.paid.2006.09.018

Baumgarten. F. (1933). Die Charaktereigenshaften. Beitrage zur Charakter und Personlichkeitsforschung: Monogr, 1. Bern: A. Francke

Benner, D. G. (1989). Toward a psychology of spirituality: Implications for personality and psychotherapy. Journal of Psychology and Christianity, 5, 19-30.

Bentler, P. M., Bonett, D. G. (1980). Significance tests and goodness of fit in the analysis of covariance structures. Psychological Bulletin, 88(3), 588 - 606. doi: 10.1037/0033-2909.88.3.588

Berdyaev, N. (1960). The origin of Russian communism. Ann Arbor: University of Michigan Press.

Bieda, A., Hirschfeld, G., Schonfeld, P., Brailovskaia, J., Zhang, X. C., \& Margraf, J. (2017). Universal happiness? Cross-cultural measurement invariance of scales assessing positive mental health. Psychological Assessment, 29(4), 408-421. doi: $10.1037 /$ pas 0000353

Blais, M. A., Lenderking, W. R., Baer, L., deLorell, A., Peets, K., Leahy, L., \& Burns, C. (1999). Development and initial validation of a brief mental health outcome 
measure. Journal of Personality Assessment, 73(3), 359-373.

doi:10.1207/S15327752JPA7303_5

Boster, J. S., \& Maltseva, K. (2006). A crystal seen from each of its vertices: European views of European national characters. Cross-Cultural Research, 40, 47-64. doi: $10.1177 / 1069397105282849$

Bourdeau, B., Hinojosa, O., Perez, E., \& Chu, K. L. (2004). Understanding the spirituality of gay Latino men: A cross-cultural validity study. Paper presented at the 2nd Annual Mid-Year Research Conference on Religion and Spirituality, Columbia, MD.

Brehm, J. \& Rahn, W. (1997). Individual-level evidence for the causes and consequences of social capital. American Journal of Political Science, 41, 999-1023. doi: $10.2307 / 2111684$

Burke, M. T., Hackney, H., Hudson, P., Miranti, J., Watts, G. A., \& Epp, L. (1999). Spirituality, religion, and CACREP curriculum standards. Journal of Counseling and Development, 77, 251-257. doi: 10.1002/j.1556-6676.1999.tb02448.x

Cangur, S. \& Ercan, I. (2015). Comparison of model fit indices used in structural equation modeling under multivariate normality. Journal of Modern Applied Statistical Methods, 14(1), 152-167. doi: 10.22237/jmasm/1430453580

Cardarelli, K. M., Vernon, S. W., Baumler, E. R., Tortolero, S., \& Low, M. (2007). Sense of control and diabetes mellitus among U.S. adults: A cross-sectional analysis. Biopsychosocial Medicine, 1. doi:10.1186/1751-0759-1-19

Carevsky, A. A. (1898). Znachenie pravoslavija v zhizni i istoricheskoj sud'be Rossii [Meaning of Russian-Orthodoxy in life and historical fate of Russia]. Retrieved from http://www.dorogadomoj.com/d40czp02.html.

Cashwell, C. S., Young, J. S., Fulton, C. L., Willis, B. T., Giordano, A., Daniel, L. W., Crockett, J., Tate, B. N. and Welch, M. L. (2013). Clinical behaviors for 
addressing religious/spiritual issues: Do we practice what we preach?. Counseling and Values, 58, 45-58. doi:10.1002/j.2161-007X.2013.00024.x

Chervyakov, V. V., Shkolnikov, V. M., Pridemore, W. A., \& McKee, M. (2002). The changing nature of murder in Russia. Social Science \& Medicine, 55, 1713-1724. doi: 10.1016/S0277-9536(01)00299-4

Cho, I. (2004). An effect of Spiritual Transcendence on fear of intimacy. Unpublished Master's thesis, Torch Trinity Graduate School of Theology, Seoul, Korea.

Clark, W. H. (1958). How do social scientists define religion? Journal of Social Psychology, 47, 143-147. doi: 10.1080/00224545.1958.9714350

Clarke, D. (2004). Neuroticism: Moderator or mediator in the relation between locus of control and depression? Personality and Individual Differences, 37(2), 245-258. doi:10.1016/j.paid.2003.08.015

Cohen, J. (1988). Statistical power analysis for the behavioral sciences $\left(2^{\text {nd }}\right.$ ed.). Hillsdale, NJ: Lawrence Erlbaum.

Cohen, S., \& Wills, T. A. (1985). Stress, social support, and the buffering hypothesis. Psychological Bulletin, 98, 310-357. doi: 10.1037/0033-2909.98.2.310

Comfrey, A. L., \& Lee, H. B. (1992). A First Course in Factor Analysis. Hillsdale, NJ: Lawrence Erlbaum Associates.

Costa, P. T., Jr., \& McCrae, R. R. (1980). Influence of extraversion and neuroticism on subjective well-being: Happy and unhappy people. Journal of Personality and Social Psychology, 38(4), 668-678. doi: 10.1037/0022-3514.38.4.668

Costa, P. T., Jr., \& McCrae, R. R. (1992). Revised NEO Personality Inventory and NEO Five-Factor Inventory: Professional Manual. Odessa, FL: Psychological Assessment Resources.

Costa, P. T., Jr., \& McCrae, R. R. (1994). “Set like plaster”? Evidence for the stability of adult personality. In T. F. Heatherton \& J. L. Weinberger (Eds.), Can personality 
change? Washington, DC: American Psychological Association. doi:

10.1037/10143-002

Costa, P. T., Jr., \& McCrae, R. R. (2002). Looking backward: Changes in the mean levels of personality traits from 80 to 12. In D. Cervone \& W. Mischel (Eds.), Advances in Personality Science (pp. 219-237). New York: Guilford Press.

Costa, P. T., McCrae, R. R., Martin, T. A., Oryol, V. E., Senin, I. G., Rukavishnikov, A. A., ... Realo, A. (2000). Personality development from adolescence through adulthood: Further cross-cultural comparisons of age differences. In V. J. Molfese \& D. Molfese (Eds.). Temperament and personality development across the lifespan (pp. 235-252). Hillsdale, NJ: Lawrence Erlbaum Associates.

Dalgard, O., \& Håheim, L. (1998). Psychosocial risk factors and mortality: A prospective study with special focus on social support, social participation, and locus of control in Norway. Journal of Epidemiology and Community Health, 52(8), 476481. doi:10.1136/jech.52.8.476

Dein, S. (2013). Religion, spirituality, depression, and anxiety: Theory, research, and practice. In K. I. Pargament, A. Mahoney, \& E. P. Shafranske (Eds.), $A P A$ handbook of psychology, religion, and spirituality (vol 2): An applied psychology of religion and spirituality (pp. 241-255). Washington, DC: American Psychological Association.

Delgado, C. (2005). A discussion on the concept of spirituality. Nursing Science Quarterly, 18, 157-162. doi:10.1177/0894318405274828

Del Rio, C. M., \& White, L. J. (2012). Separating spirituality from religiosity: A hylomorphic attitudinal perspective. Psychology of Religion and Spirituality, 4(2), 123-142. doi:10.1037/a0027552

Digman, J. M. (1990). Personality structure: Emergence of the five-factor model. Annual Review of Psychology, 41, 417-440. doi:10.1146/annurev.ps.41.020190.002221 
Dinesen, P. (2012). Does generalized (dis)trust travel? Examining the impact of cultural heritage and destination-country environment on trust of immigrants. Political Psychology, 33(4), 495-511. doi:10.1111/j.1467-9221.2012.00886.x

Doyle, D. (1992). Have we looked beyond the physical and psychosocial? Journal of Pain and Symptom Management, 7, 302-311. doi: 10.1016/0885-3924(92)90063$\mathrm{N}$

Dragomirecka, E., Lenderking, W. R., Motlova, L., Goppoldova, E., \& Šelepova, P. (2006). A brief mental health outcomes measure: Translation and validation of the Czech version of the Schwartz Outcomes Scale-10. Quality of Life Research: An International Journal of Quality of Life Aspects of Treatment, Care \& Rehabilitation, 15(2), 307-312. doi:10.1007/s11136-005-1389-y

Emadpoor, L., Lavasani, M. G., \& Shahcheraghi, S. M. (2016). Relationship between perceived social support and psychological well-being among students based on mediating role of academic motivation. International Journal Of Mental Health And Addiction, 14(3), 284-290. doi:10.1007/s11469-015-9608-4

Elam, D. A. (2001, January). An exploration of the relationship between spirituality and emotional well-being. Dissertation Abstracts International, 62, 2955.

Faul, F., Erdfelder, E., Buchner, A., \& Lang, A. G. (2009). Statistical power analyses using $\mathrm{G}^{*}$ Power 3.1: Tests for correlation and regression analyses. Behavior Research Methods, 41, 1149-1160. doi: 10.3758/BRM.41.4.1149

Field, A. (2009). Discovering statistics using SPSS (3 ${ }^{\text {rd }}$ ed.). Thousand Oaks, CA, US: Sage Publications, Inc.

Figes, O. (2003). Natasha's dance: A cultural history of Russia. New York: Picador.

Froese, P. (2004). Forced secularization in Soviet Russia: Why an atheistic monopoly failed. Journal for the Scientific Study of Religion, 43(1), 35-50. doi:10.1111/j.1468-5906.2004.00216.x 
Fuller-Iglesias, H. R. (2015). Social ties and psychological well-being in late life: The mediating role of relationship satisfaction. Aging \& Mental Health, 19(12), 11031112. doi:10.1080/13607863.2014.1003285

Fuller-Thomson, E., Agbeyaka, S., LaFond, D. M., \& Bern-Klug, M. (2016). Flourishing after depression: Factors associated with achieving complete mental health among those with a history of depression. Psychiatry Research, 242, 111-120. doi: 10.1016/j.psychres.2016.04.041

Gale, C. R., Batty, G., \& Deary, I. J. (2008). Locus of control at age 10 years and health outcomes and behaviors at age 30 years: The 1970 British Cohort Study. Psychosomatic Medicine, 70(4), 397-403. doi:10.1097/PSY.0b013e31816a719e

Geis, K.J., \& Ross, C.E. (1998). A new look at urban alienation: The effect of neighborhood disorder on perceived powerlessness. Social Psychology Quarterly, $61(3), 232-246$.

Gerber, T. P., \& Mendelson, S. E. (2008). Public experiences of police violence and corruption in contemporary Russia: A case of predatory policing?. Law \& Society Review, 42(1), 1-44. doi:10.1111/j.1540-5893.2008.00333.x

Goodman, J. M. (2002). Psychological well-being in the Jewish community: The impact of social identity and spirituality. Unpublished doctoral dissertation, Kent State University.

Gore, J. S., Griffin, D. P., \& McNierney, D. (2016). Does internal or external locus of control have a stronger link to mental and physical health? Psychological Studies, 61(3),181-196. doi: 10.1007/s12646-016-0361-y

Gough, H. G. (2000). Adjective Checklist. In A. E. Kazdin (Ed.), Encyclopedia of psychology, Vol. 1 (pp. 36-37). Washington, DC New York, NY USUS: American Psychological Association. doi:10.1037/10516-014

Gough, H. G., \& Heilbrun, A. B. (1983). The Adjective Check List Manual. (1983 ed.). Palo Alto, CA: Consulting Psychologists Press. 
Greenway, A., Phelan, M., Turnbull, S., \& Milne, L. C. (2007). Religious coping strategies and spiritual transcendence. Mental Health, Religion \& Culture, 10(4), 325-333. doi:10.1080/13694670600719839

Grob, A., Little, T. D., Wanner, B., Wearing, A. J., \& Euronet. (1996). Adolescents' wellbeing and perceived control across 14 sociocultural contexts. Journal of Personality and Social Psychology, 71, 785-795. doi: 10.1037/00223514.71.4.785

Haggerty, G., Blake, M., Naraine, M., Siefert, C., \& Blais, M. A. (2010). Construct validity of the Schwartz Outcome Scale-10: Comparisons to interpersonal distress, adult attachment, alexithymia, the Five Factor Model, romantic relationship length and ratings of childhood memories. Clinical Psychology \& Psychotherapy, 17(1), 44-50.

Haggerty, G., Blake, M., \& Siefert, C. J. (2010). Convergent and divergent validity of the relationship profile test: Investigating the relationship with attachment, interpersonal distress, and psychological health. Journal of Clinical Psychology, 66(4), 339-354. doi: 10.1002/jclp.20654

Hair, J. F., Jr., Black, W. C., Babin, B. J., \& Anderson, R. E. (2010). Multivariate data analysis $\left(7^{\text {th }}\right.$ ed.). Upper Saddle River, NJ: Prentice Hall.

Heleniak, T. (1995). Economic transition and demographic change in Russia, 1989-1995. Post-Soviet Geography 36, 446-458.

Hill, T., D., Ross, C. E., \& Angel, R. J. (2005). Neighborhood disorder, psychophysiological distress, and health. Journal of Health and Social Behavior, 46, 170-186. doi: 10.1177/002214650504600204

Howard, M. C. (2016) A review of exploratory factor analysis decisions and overview of current practices: What we are doing and how can we improve? International Journal of Human-Computer Interaction, 32(1), 51-62. doi: $10.1080 / 10447318.2015 .1087664$ 
Hu, L., \& Bentler, P. M. (1999). Cutoff criteria for fit indexes in covariance structure analysis: Conventional criteria versus new alternatives. Structural Equation Modeling, 6(1), 1-55. doi: 10.1080/10705519909540118

Joe, V. C. (1971). Review of the internal-external control construct as a personality variable. Psychological Reports, 28(2), 619-640. doi: 10.2466/pr0.1971.28.2.619

John. O. P. (1990). The "Big Five" factor taxonomy: Dimensions of personality in the natural language and in questionnaires. In L. Pervin (Ed.), Handbook of personality theory and research (pp. 66-100). New York: Guilford.

John, O. P., Donahue, E. M., \& Kentle, R. L. (1991). The Big Five Inventory-Versions $4 a$ and 54. Berkeley, CA: University of California, Berkeley, Institute of Personality and Social Research.

John, O. P., \& Srivastava, S. (1999). The Big Five trait taxonomy: History, measurement, and theoretical perspectives. In L. A. Pervin \& O. P. John (Eds.), Handbook of personality theory and research (pp. 102-138). New York: Guilford Press.

Judge, T. A., Erez, A., Bono, J. E., \& Thoresen, C. J. (2002). Are measures of selfesteem, neuroticism, locus of control, and generalized self-efficacy indicators of a common core construct?. Journal of Personality and Social Psychology, 83(3), 693-710. doi:10.1037/0022-3514.83.3.693

Kawachi, I., \& Berkman, L. F. (2001). Social ties and mental health. Journal of Urban Health: Bulletin of the New York Academy of Medicine, 78(3), 458-467. doi: 10.1093/jurban/78.3.458

Kenny, D. A. (2015). Measuring model fit. Retrieved from http://davidakenny.net/cm/fit.htm

Kim, J., \& Conley, M. E. (2011). Neighborhood disorder and the sense of personal control: Which factors moderate the association?. Journal of Community Psychology, 39(8), 894-907. doi:10.1002/jcop.20476 
Kim, S., \& Pridemore, W. (2005). Social change, institutional anomie and serious property crime in transitional Russia. British Journal of Criminology, 45(1), 8197. doi:10.1093/bjc/azh082

Klages, L. (1926). The science of character. (Transl. 1932). London: Allen \& Unwin

Kline, R. B. (2011). Principles and practice of structural equation modeling $\left(3^{\text {rd }} \mathrm{ed}.\right)$. New York: Guilford Press.

Koenig, H. G. (2012). Religion, spirituality, and health: The research and clinical implications. ISRN Psychiatry, 2012, 1-33. doi: 10.5402/2012/278730

Konick, L. C. (2009). Evaluation of spirituality as a moderator of risk for suicidal ideation in a community sample of adolescents. Dissertation Abstracts International, 70, 3787.

Lachman, M. E., \& Weaver, S. L. (1998). The sense of control as a moderator of social class differences in health and well-being. Journal of Personality and Social Psychology, 74(3), 763-773. doi:10.1037/0022-3514.74.3.763

Latkin, C., \& Curry, A. (2003). Stressful neighborhoods and depression: A prospective study of the impact of neighborhood disorder. Journal of Health and Social Behavior, 44, 34-44. doi: 10.2307/1519814

Laux, J. M., \& Ahern, B. (2003). Concurrent validity of the Schwartz Outcome Scale with a chemically dependent population. Journal of Addictions \& Offender Counseling, 24(1), 2-10. doi:10.1002/j.2161-1874.2003.tb00177.x

Marsh, H. W., \& Hau, K. T. (1996). Assessing goodness of fit: Is parsimony always desirable? The Journal of Experimental Education, 64, 364-390. doi: 10.1080/00220973.1996.10806604

Marsh, H. W., Hau, K., \& Wen, Z. (2004). In Search of Golden Rules: Comment on Hypothesis-Testing Approaches to Setting Cutoff Values for Fit Indexes and Dangers in Overgeneralizing Hu and Bentler's (1999) Findings. Structural Equation Modeling, 11(3), 320-341. doi:10.1207/s15328007sem1103_2 
Martin, T. A., Costa, P. T., Oryol, V. E., Rukavishnikov, A. A., \& Senin, I. G. (2002). Applications of the Russian NEO-PI-R. In R. R. McCrae \& J. Allik (Eds.), The Five-Factor Model of personality across cultures (pp. 261-277). New York, NY: Kluwer Academic/Plenum Publishers.

McCrae, R. R. (1996). Social consequences of experiential openness. Psychological Bulletin, 120, 323-337. doi:10.1037/0033-2909.120.3.323

McCrae, R. R. (2002). NEO-PI-R data from 36 cultures. In R. R. McCrae and J. Allik (eds.). The Five-Factor model of personality across cultures (pp. 105-125). New York: Kluwer Academic/Plenum Publishers.

McCrae, R. R., \& Costa, P. T., Jr. (1985). Updating Norman's “Adequate Taxonomy”: Intelligence and personality dimensions in natural language and in questionnaires. Journal of Personality and Social Psychology, 49, 710-721. doi: 10.1037/00223514.49.3.710

McCrae, R. R. \& Costa, P. T., Jr. (1986). Personality, coping, and coping effectiveness in an adult sample. Journal of Personality, 54, 385-405. doi: 10.1111/j.14676494.1986.tb00401.x

McCrae, R. R., \& Costa, P. T., Jr. (1987). Validation of the five-factor model of personality across instruments and observers. Journal of Personality and Social Psychology, 52, 81-90. doi: 10.1037/0022-3514.52.1.81

McCrae, R. R., \& Costa, P. T. (1992). Discriminant validity of NEO-PIR facet scales. Educational and Psychological Measurement, 52(1), 229-237. doi:10.1177/001316449205200128

McCrae, R. R., \& Costa, P. T., Jr. (2003). Personality in adulthood: A five-factor theory perspective ( $2^{\text {nd }}$ Ed.). New York, NY: Guilford Press.

McCrae, R. R., Gaines, J. F., \& Wellington, M. A. (2013). The Five-Factor Model in fact and fiction. In H. Tennen, J. Suls, I. B. Weiner (Eds.), Handbook of psychology, 
Vol. 5: Personality and social psychology (2nd ed.) (pp. 65-91). Hoboken, NJ US: John Wiley \& Sons Inc.

McCrae, R. R., Terracciano, A. and 78 Members of the Personality Profiles of Cultures Project (2005). Universal features of personality traits from the observer's perspective: Data from 50 cultures. Journal of Personality and Social Psychology, 88(3), 547-561. doi: 10.1037/0022-3514.88.3.547

McDougall, W. W. (1932). Of the words character and personality. Character \& Personality; A Quarterly for Psychodiagnostic \& Allied Studies, 13-16. doi: 10.1111/j.1467-6494.1932.tb02209.x

McElroy, E. M. (1999). The effect of God image and religious coping on depression, well-being and alcohol use in college students. Dissertation Abstracts International, 60, 1862.

Meyers, L. S., Gamst, G., \& Guarino, A. J. (2006). Applied multivariate research: Design and interpretation. SAGE Publications, Incorporated.

Mirowsky, J., \& Ross, C. E. (1983). Paranoia and the structure of powerlessness. American Sociological Review, 48(2), 228-239. doi: 10.2307/2095107

Mirowsky, J., \& Ross, C.E. (1990). Control or defense-Depression and the sense of control over good and bad outcomes. Journal of Health and Social Behavior, 31(1), 71-86. doi: 10.2307/2137046

Mirowsky, J., \& Ross, C. E. (1991). Eliminating defense and agreement bias from measures of the sense of control: A $2 \times 2$ index. Social Psychology Quarterly, 54(2), 127-145. doi:10.2307/2786931

Mirowsky, J., \& Ross, C. E. (2003). Social Causes of Psychological Distress. 2nd ed. New Brunswick, NJ: Aldine Transaction.

Norman, W. T. (1963). Toward an adequate taxonomy of personality attributes: Replicated factor structure in peer nomination personality ratings. The Journal of Abnormal and Social Psychology, 66(6), 574-583. doi:10.1037/h0040291 
Nemtsov, A.V. (2003). Suicide and alcohol consumption in Russia, 1965-1999. Drug and Alcohol Dependence, 1, 161-168. doi: 10.1016/S0376-8716(03)00094-2

Otani, A. (2002). When science meets religion. American Behavioral Scientist, 45, 19021904. doi: $10.1177 / 0002764202045012012$

Paine, D. R. \& Sandage, S. J. (2017). Religious involvement and depression: The mediating effect of relational spirituality. Journal of Religion and Health, 56(1), 269-283. doi: 10.1007/s10943-016-0282-z

Pargament, K. I. (1999). The psychology of religion and spirituality? Yes and no. International Journal for the Psychology of Religion, 9(1), 3-16. doi:10.1207/s15327582ijpr0901_2

Pargament, K. I., Smith, B. W., Koenig, H. G., \& Perez, L. (1998). Patterns of positive and negative religious coping with major life stressors. Journal of the Scientific Study of Religion, 37(4), 710-724. doi: 10.2307/1388152

Pargament, K. I., Koenig, H. G., \& Perez, L. M. (2000). The many methods of religious coping: Development and initial validation of the RCOPE. Journal of Clinical Psychology, 56, 519-543. doi: 10.1002/(SICI)1097-4679(200004)56:4<519::AIDJCLP6>3.0.CO;2-1

Peabody, D., \& Shmelyov, A. G. (1996). Psychological characteristics of Russians. European Journal of Social Psychology, 26(3), 507-512. doi:10.1002/(SICI)10990992(199605)26:3<507::AID-EJSP762>3.0.CO;2-A

Pearlin, L. I., Menaghan, E. G., Lieberman, M. A., \& Mullan, J. T. (1981). The stress process. Journal of Health and Social Behavior, 22, 337-356. doi: $10.2307 / 2136676$

Piedmont, R. L. (1999). Does spirituality represent the sixth factor of personality? Spiritual Transcendence and the Five-Factor Model. Journal of Personality, 67(6), 985-1013. doi:10.1111/1467-6494.00080 
Piedmont, R. L. (2001). Spiritual Transcendence and the scientific study of spirituality. Journal of Rehabilitation, 67, 4-14.

Piedmont, R. L. (2004). Assessment of Spirituality and Religious Sentiments, technical manual. Baltimore, MD; Author.

Piedmont, R. L. (2007). Cross-cultural generalizability of the Spiritual Transcendence Scale to the Philippines: Spirituality as a human universal. Mental Health, Religion \& Culture, 10(2), 89-107. doi:10.1080/13694670500275494

Piedmont, R. L. (2012). Overview and development of a trait-based measure of numinous constructs: The Assessment of Spirituality and Religious Sentiments (ASPIRES) Scale. In L. J. Miller (Ed.), The Oxford handbook of psychology and spirituality (pp. 104-122). New York, NY, US: Oxford University Press. doi:10.1093/oxfordhb/9780199729920.013.0007

Piedmont, R. L., Ciarrochi, J. W., Dy-Liacco, G. S., \& Williams, J. G. (2009). The empirical and conceptual value of the Spiritual Transcendence and religious involvement scales for personality research. Psychology of Religion and Spirituality, 1(3), 162-179. doi:10.1037/a0015883

Piedmont, R. L., \& Leach, M. M. (2002). Cross-cultural generalizability of the Spiritual Transcendence Scale in India: Spirituality as a universal aspect of human experience. American Behavioral Scientist, 45(12), 1888-1901. doi:10.1177/0002764202045012011

Price, R. H. (2003). Commentary: Personal control: Mediation, measurement, and malleability. In S. H., Zarit, L. I. Pearlin, \& K. W. Schaie (Eds.), Personal control in social and life course contexts (pp. 165-172). New York, NY: Springer.

Pridemore, W. (2002). Social problems and patterns of juvenile delinquency in transitional Russia. Journal of Research in Crime and Delinquency, 39(2), 187213. doi:10.1177/002242780203900203 
Pridemore, W. A. (2003). Demographic, temporal, and spatial patterns of homicide rates in Russia. European Sociological Review, 19, 41-59. doi: 10.1093/esr/19.1.41

Pridemore, W. A. (2006). Heavy Drinking and Suicide in Russia. Soc Forces, 85(1), $413-$ 430. 10.1353/sof.2006.0138

Pridemore, W.A., Spivak, A.L. (2003). Pattern of suicide mortality in Russia. Suicide and Life-Threating Behavior, 33(2), 132-150. doi: 10.1521/suli.33.2.132.22771

Pudrovska, T., Schieman, S., Pearlin, L. I., \& Nguyen, K. (2005). The sense of mastery as a mediator and moderator in the association between economic hardship and health in late life. Journal of Aging and Health, 17(5), 634-660. doi:10.1177/0898264305279874

Rammstedt, B., \& John, O. P. (2007). Measuring personality in one minute or less: A 10item short version of the Big Five Inventory in English and German. Journal of Research in Personality, 41(1), 203-212. doi:10.1016/j.jrp.2006.02.001

Razvodovsky, Y. E. (2008). A comparative analysis ofdynamic of suicides in Belarus and Russia. Journal of Belarusian Psychiatric Association, 14, 15-19.

Razvodovsky, Y. E. (2010). Beverage-specific alcohol sales and violent mortality in Russia. Adicciones, 22(4), 311-316. doi: 10.20882/adicciones. 172

Razvodovsky, Y. E. (2011). Alcohol consumption and suicide rates in Russia. Suicidology Online, 2, 67-74.

Realo, A., Allik, J., Lönnqvist, J., Verkasalo, M., Kwiatkowska, A., Kööts, L., \& ... Renge, V. (2009). Mechanisms of the national character stereotype: How people in six neighbouring countries of Russia describe themselves and the typical Russian. European Journal of Personality, 23(3), 229-249. doi:10.1002/per.719

Reed, T. D., \& Neville, H. A. (2014). The influence of religiosity and spirituality on psychological well- being among Black women. Journal of Black Psychology, 40, 384-401. doi: 10.1177/0095798413490956 
Reeskens, T., \& Hooghe, M. (2008). Cross-cultural measurement equivalence of generalized trust. Evidence from the European Social Survey (2002 and 2004). Social Indicators Research, 85(3), 515-532. doi:10.1007/s11205-007-9100-Z

Rehm, J., Taylor, B., Patra, J. (2006). Volume of alcohol consumption, pattern of drinking and burden of disease in the European region. Addiction, 101, 1086-95. doi: 10.1111/j.1360-0443.2006.01491.x

Reutter, K. K. (2013). The effects of spirituality and religiosity upon stress, anxiety, and depression: Mediation, moderation, or moderated mediation? Dissertation Abstracts International: Section B: The Sciences and Engineering, 73, 11-B(E).

Reutter, K. K., \& Bigatti, S. M. (2014). Religiosity and spirituality as resiliency resources: Moderation, mediation, or moderated mediation? Journal for the Scientific Study of Religion, 53(1), 56-72. doi: 10.1111/jssr.12081

Ribaudo, A., \& Takahashi, M. (2008). Temporal trends in spirituality research: A metaanalysis of journal abstracts between 1944 and 2003. Journal of Religion, Spirituality \& Aging, 20(1-2), 16-28. doi:10.1080/15528030801921972

Robertson, A. (2006). The significance of language, culture, and communication in researching post-Soviet crime and policing. Journal of Contemporary Criminal Justice, 22(2), 137-156. doi:10.1177/1043986206286931

Rolland, J. P. (2002). The cross-cultural generalizability of the Five Factor Model of personality. In R. R. McCrae and J. Allik (eds.). The Five-Factor model of personality across cultures (pp. 7-28). New York: Kluwer Academic/Plenum Publishers.

Rose, E. M., Westefeld, J. S., \& Ansely, T. N. (2001). Spiritual issues in counseling: Clients' beliefs and preferences. Journal of Counseling Psychology, 48(1), 61-71. http://dx.doi.org/10.1037/0022-0167.48.1.61

Ross, C. E. (2000). Neighborhood disadvantage and adult depression. Journal of Health and Social Behavior, 41(2), 177-187. doi:10.2307/2676304 
Ross, C. E. (2011). Collective threat, trust, and the sense of personal control. Journal of Health and Social Behavior, 52(3), 287-296. doi:10.1177/0022146511404558

Ross, C. E., \& Mirowsky, J. (1999). Disorder and decay: The concept and measurement of perceived neighborhood disorder. Urban Affairs Review, 34(3), 412-432. doi: $10.1177 / 107808749903400304$

Ross, C. E., \& Mirowsky, J. (2002). Age and the gender gap in the sense of personal control. Social Psychology Quarterly, 65(2), 125-145. doi:10.2307/3090097

Ross, C. E., \& Mirowsky, J. (2003). Social structure and psychological functioning: Distress, perceived control, and trust. In J. Delamater (Ed.), Handbook of social psychology (pp. 411-447). New York, NY US: Kluwer Academic/Plenum Publishers.

Ross, C. E., \& Mirowsky. J. (2009). Neighborhood disorder, subjective alienation, and distress. Journal of Health and Social Behavior, 50, 49-64. doi: $10.1177 / 002214650905000104$

Ross, C. R., Mirowsky, J., \& Pribesh, S. (2001). Powerlessness and the amplification of threat: Neighborhood disadvantage, disorder, and mistrust. American Sociological Review, 66, 568-91. doi: 10.2307/3088923

Ross, C. R., Mirowsky, J., \& Pribesh, S. (2002). Disadvantage, disorder, and urban mistrust. City \& Community, 1(1), 59-82. doi:10.1111/1540-6040.00008

Ross, C, E., Reynolds, J. R., \& Geis, K. J. (2000). The contingent meaning of neighborhood stability for residents' psychological well-being. American Sociological Review, 65, 581-97. doi: 10.2307/2657384

Rotter, J. B. (1966). Generalized expectancies for internal vs. external control of reinforcements. Psychological Monographs, 80, 1-28. doi: 10.1037/h0092976

Rotter, J. B. (1980). Interpersonal trust, trustworthiness, and gullibility. American Psychologist, 35, 1-7. doi: 10.1037/0003-066X.35.1.1 
Rotter, J. B. (1990). Internal versus external control of reinforcement: A case history of a variable. American Psychologist, 45(4), 489-493. doi: 10.1037/0003066X.45.4.489

Salsman, J. M., Pustejovsky, J. E., Jim, H. L., Munoz, A. R., Merluzzi, T. V., George, L., \& ... Fitchett, G. (2015). A meta-analytic approach to examining the correlation between religion/spirituality and mental health in cancer. Cancer, 121(21), 37693778. doi:10.1002/cncr.29350

Sandage, S. J., \& Jankowski, P. J. (2010). Forgiveness, spiritual instability, mental health symptoms, and well-being: Mediation effects of differentiation of self. Psychology of Religion and Spirituality, 2, 168-180. doi: 10.1037/a0019124

Schieman, S., \& Meersman, S. C. (2004). Neighborhood problems and health among older adults: Received and donated social support and the sense of mastery as effect modifiers. Journal of Gerontology: Social Sciences, 59, 89-97. doi: 10.1093/geronb/59.2.S89

Shippee, N. D. (2012). Victimization, fear of crime, and perceived risk: Testing a vulnerability model of personal control. Sociological Perspectives, 55(1), 117 140. doi:10.1525/sop.2012.55.1.117

Schulz, A., Williams, D., Israel, B., Becker, A., Parker, E., James, S., \& Jackson, J. (2000). Unfair treatment, neighborhood effects, and mental health in the Detroit metropolitan area. Journal of Health and Social Behavior, 41, 314-32. doi: $10.2307 / 2676323$

Seeman, M. (1959). On the meaning of alienation. American Sociological Review, 24, 782-791. doi: $10.2307 / 2088565$

Seeman, M. (1983). Alienation motifs in contemporary theorizing: The hidden continuity of classic themes. Social Psychology Quarterly, 46, 171-84. doi: $10.2307 / 3033789$ 
Seeman, M., \& Lewis, S. (1995). Powerlessness, health and mortality: A longitudinal study of older men and mature women. Social Science \& Medicine, 41(4), 517525. doi:10.1016/0277-9536(94)00362-W

Semenova, K. (2015, April 7). A new emigration: The best are leaving. Part 1. Institute of Modern Russia. Retrieved from http://imrussia.org

Sharmaa, S., Mukherjeeb, S., Kumarc, A., \& Dillon, W. R. (2005). A simulation study to investigate the use of cutoff values for assessing model fit in covariance structure models. Journal of Business Research, 58, 935-943. doi:

10.1016/j.jbusres.2003.10.007

Slater, W., Hall, T. W., \& Edwards, K. J. (2001). Measuring religion and spirituality: Where are we and where are we going? Journal of Psychology and Theology, 29(1), 4-21.

Slobodskaya, H. R. (2007). The associations among the big five, behavioural inhibition and behavioural approach systems and child and adolescent adjustment in Russia. Personality and Individual Differences, 43, 913-924. doi:

10.1016/j.paid.2007.02.012

Smith, T. W. (1988). The ups and downs of cross-national survey research. GSS CrossNational Report No. 8. Chicago: National Opinion Research Center, University of Chicago.

Staton-Tindall, M., Duvall, J., Stevens-Watkins, D., \& Oser, C. B. (2013). The roles of spirituality in the relationship between traumatic life events, mental health, and drug use among African American women from one southern state. Substance Use \& Misuse, 48(12), 1246-1257. doi:10.3109/10826084.2013.799023

Steptoe, A., \& Feldman, P. (2001). Neighborhood problems as sources of chronic stress: Development of a measure of neighborhood problems, and association with socioeconomic status and health. Annals of Behavioral Medicine, 23, 177-85. doi: 10.1207/S15324796ABM2303_5 
Stevens, J. P. (2009). Applied multivariate statistics for the social sciences $\left(5^{\text {th }} \mathrm{ed}\right.$.). New York, NY: Taylor \& Francis Group, LLC.

Tabachnick, B. G., \& Fidell, L. S. (2007). Using multivariate statistics ( $5^{\text {th }}$ ed.). Boston, MA: Pearson.

Tan, S., \& Tambyah, S. (2011). Generalized trust and trust in institutions in Confucian Asia. Social Indicators Research, 103(3), 357-377. doi:10.1007/s11205-0109703-7

Terracciano, A., Abdel-Khalek, A. M., Adam, N., Adamovova, L., Ahn, C., Ahn, H. N., et al. (2005). National character does not reflect mean personality trait levels in 49 cultures. Science, 310(5745), 96-100. doi: 10.1126/science.1117199

Terracciano, A., \& McCrae, R. R. (2007). Implications for understanding national character stereotypes. Journal of Cross-Cultural Psychology, 38, 695-710. doi: $10.1177 / 0022022107308586$

Tupes, E. C., \& Christal, R. E. (1992). Recurrent personality factors based on trait ratings. Journal of Personality, 60(2), 225-251. doi:10.1111/j.14676494.1992.tb00973.x

Uma, G. (2016). Age, mental health and well-being. Journal of Psychological Research, 11(1), 147-156.

Vaughan, F. (1991). Spiritual issues in psychotherapy. Journal of Transpersonal Psychology, 23, 105-119.

Verma, J., \& Triandis, H. C. (1999). The measurement of collectivism in India. In W. J. Lonner, D. L. Dinnel, D. K. Forgays, S. A. Hayes (Eds.), Merging past, present, and future in cross-cultural psychology: Selected papers from the Fourteenth International Congress of the International Association for Cross-Cultural Psychology (pp. 256-265). Lisse, Netherlands: Swets \& Zeitlinger Publishers.

Ward, M. M. (2013). Sense of control and self-reported health in a population-based sample of older Americans: Assessment of potential confounding by affect, 
personality, and social support. International Journal of Behavioral Medicine, 20(1), 140-147. doi:10.1007/s12529-011-9218-x

Weaver, A. J., Pargament, K. I., Flannelly, K. J., \& Oppenheimer, J. E. (2006). Trends in the scientific study of religion, spirituality, and health: 1965-2000. Journal of Religion and Health, 45(2), 208-214. doi:10.1007/s10943-006-9011-3

Weid, C. \& Eriksen, K. (2007). Christian clients' preferences regarding prayer as a counseling intervention. Jouran of Psychology and Theology, 35(4), 328-341.

Weinberg, M. (2017). Trauma and social support: The association between informal social support, formal social support, and psychological well-being among terror attack survivors. International Social Work, 60(1), 208-218. doi:10.1177/0020872814564704

Weiss, D., Freund, A. M., \& Wiese, B. S. (2012). Mastering developmental transitions in young and middle adulthood: The interplay of openness to experience and traditional gender ideology on women's self-efficacy and subjective well-being. Developmental Psychology, 48(6), 1774-1784. doi:10.1037/a0028893

Wheaton, B. (1980). The sociogenesis of psychological disorder: An Attributional Theory. Journal of Health and Social Behavior, 21, 100-124. doi: $10.2307 / 2136730$

Wilson, T. (2004). Ethnic identity and spirituality in the recovery from alcoholism among aboriginal Canadians. Unpublished Masters thesis, University of Windsor.

Wong-MacDonald, A., \& Gorsuch, R. L. (2000). Surrender to God: An additional coping style? Journal of Psychology and Theology, 28, 149-161.

World Prison Brief (2011), available online at http://www.prisonstudies.org/info/worldbrief/.

Yedlin, J. I. (2013). The role of religion and spirituality in the relation between trauma exposure, posttraumatic stress disorder, and alcohol use in emerging adults. Dissertation Abstracts International, 73. 
Young, J. L., Waehler, C. A., Laux, J. M., McDaniel, P., \& Hilsenroth, M. J. (2003). Four studies extending the utility of the Schwartz outcome scale (SOS-10). Journal of Personality Assessment, 80(2), 130-138. doi:10.1207/S15327752JPA8002_02

Zieger, C. E. (2008). Russia and the CIS in 2007: Putin's final year? Asian Survey, 48(1), 133-143. doi: 10.1525/as.2008.48.1.133

Zinnbauer, B. J., Pargament, K. I., Cole, B., Rye, M. S., Butter, E. M., Belavich, T. G., \& ... Kadar, J. L. (1997). Religion and spirituality: Unfuzzying the fuzzy. Journal for the Scientific Study of Religion, 36(4), 549-564. doi:10.2307/1387689

Zmerli, S., \& Newton, K. (2008). Social trust and attitudes toward democracy. Public Opinion Quarterly, 72(4), 706-724. doi:10.1093/poq/nfn054 


\section{CURRICULUM VITAE Ksenia Kopeikin}

52 Highland Avenue, Everett, MA 02149

ksenia.kopeikin@gmail.com

(617) 945-4597

\section{EDUCATION}

\begin{tabular}{lll}
\hline Aug 2011 - & UNIVERSITY OF LOUISVILLE & Louisville, KY \\
Aug 2017 & $\begin{array}{l}\text { Ph.D. in Counseling \& Personnel Services (Counseling Psychology) } \\
\text { Dissertation: Cross-Cultural Generalizability of the Spiritual Transcendence in Russia } \\
\text { Adviser: Mark M. Leach, Ph.D. }\end{array}$ & Norman, OK \\
June 2009 - & UNIVERSITY OF OKLAHOMA & \\
May 2011 & Master of Education in Community Counseling & Columbia, MO \\
Aug 2005 - & $\begin{array}{l}\text { UNIVERSITY OF MISSOURI - COLUMBIA } \\
\text { May 2009 }\end{array}$ & $\begin{array}{l}\text { Bachelor of Arts in Psychology } \\
\text { Minor in Human Development and Family Studies }\end{array}$
\end{tabular}

\section{SCHOLARLY ACTIVITY}

- Peterson, D.J., Gargya, S., Kopeikin, K.S., \& Naveh-Benjamin, M. (in press). The impact of level of education/ability on age-related deficits in associative memory: Behavioral and neuropsychological perspectives. Manuscript submitted for publication.

- Peters, S.A., \& Kopeikin, K.S. (2016). Integrated reasoning about statistical variation: Secondary teachers' development of foundational understandings. In D. Ben-Zvi, \& K. Makar. (Eds.), The teaching and learning of statistics: International perspectives (pp. 245-254).

- Latino, E. R., Kopeikin, K.S., \& Leach, M. M. (2014). A cross-sectional analysis of international ethics codes focusing on multiple relationships. Poster presented at the 2014 Counseling Psychology Conference. Atlanta, GA.

- $\quad$ Kopeikin, K.S., Latino, E.R., \& Leach, M.M. (2014). An examination of social justice standards found in international ethics codes. Poster presented at the Counseling Psychology Conference, 2014. Atlanta, GA.

- $\quad$ Leach, M.M., \& Kopeikin, K.S. (February 20, 2013). The long arms of counseling psychology. [Review of the book The APA handbook of counseling psychology]. PsycCRITIQUES - Contemporary Psychology: APA Review of Books, 58, 8, Article 2.

- Kopeikin, K. S., Latino, E. R., \& Leach, M. M. (2012). A Cross-Sectional Analysis of International Psychological Ethics Codes. Poster presented at the Great Lakes Counseling Psychology Conference, 2012. West Lafayette, IN. 
- Kopeikin, K. S., Talley, A. E., \& Bettencourt, B. A. (2009). The benefits of cross-sex friendship for women. Poster presented at the 2009 Midwestern Psychological Association Conference. Chicago, IL.

- Kopeikin, K. S., \& Naveh-Benjamin, M. (2007). The effects of level of education/ability on associative memory deficits in older adults. Manuscript submitted to the Arts and Science Undergraduate Research Mentor Program.

\section{CLINICAL EXPERIENCE}

Aug 2015 -

Aug 2016

Aug 2013 -

May 2014

Aug 2012 May 2013

June 2010 May 2011

Aug 2009 May 2010

June 2008 Dec 2008
DOCTORAL INTERNSHIP Suffolk University Counseling, Health \& Wellness Supervised by Teresa Blevins, Ph.D. and Jean Joyce-Brady, Ph.D.

- Provided individual therapy to the student population at Suffolk University

- Provided outreach services, including RA Training and National Depression Screening Day

- Worked as a liaison with the International Student Office

- Provided individual supervision to a Practicum student in their first year of clinical service

- Acquired a total of 514 direct client contact hours

Advanced Practicum in Counseling Bellarmine University Counseling Center Supervised by Amy Wendell, Psy.D.

- Provided individual therapy to the student population at Bellarmine University

- Provided outreach services

- Worked as a liaison with the Academic Resources Center

- Acquired a total of 226 direct client contact hours

Advanced Practicum in Counseling Seven Counties Services, Inc., Louisville KY Supervised by Elizabeth Jackson, Ph.D.

- Provided individual, family and group therapy to the local community population

- Acquired a total of 162 direct client contact hours

COMMUNITY COUNSELING INTERNSHIP

Crossroads Youth \& Family, Norman OK Supervised by Mike Templeton, M.Ed.

- Provided individual, couples and group therapy at the agency and in Norman Public Schools

- Lead psychoeducational groups - Directions in Divorce (DID), Alternative to Suspension Program (ASP), Teens and Parents in Partnership (TAPP)

- Acquired a total of 214 direct client contact hours

Community Counseling Practicum

University of Oklahoma Supervised by Terry Pace, Ph.D.

- Provided individual and couples therapy to the local community population at the $\mathrm{OU}$ Counseling Clinic

- Acquired a total of 62 direct client contact hours

MiD-Missouri CRISIS LiNe

Columbia, MO Volunteer

- Took phone calls from 20-county area

- Used crisis intervention V-model to talk callers through crisis situations

- Used TTY to communicate with deaf callers

- Provided necessary resources for callers in crisis 


\section{RESEARCH AND APPLIED EXPERIENCE}

Sep 2011 -

Aug 2013

Sep 2011 -

Dec 2012

Aug 2011 -

June 2014

Aug 2011 -

July 2013

Aug 2008 -

May 2009

Jan 2008 -

May 2008

June 2007 Aug 2007

Jan 2007 -

Dec 2007
InTERNATIONAL ETHICS RESEARCH PROJeCT

University of Louisville

Supervised by Mark Leach, Ph.D.

- Coded professional ethics codes of 47 countries using content analysis

- Established adequate inter-rater reliability

- Presented three sets of data analyses at Great Lakes Counseling Psychology Conference, March 2012 and Counseling Psychology Conference, March 2014.

First-YeAR RESEARCH PROJECT

University of Louisville Supervised by Mark Leach, Ph.D.

- Reviewed the literature regarding forgiveness in long-distance relationships

- Developed hypotheses and measures for the variables under study

- Collected and analyzed data

GRADUATE RESEARCH ASSISTANT

University of Louisville Supervised by Susan Peters, Ph.D.

- Assisted with coding and analysis of qualitative data regarding influential learning experiences for statistics teachers

- Reviewed and summarized research literature regarding math and statistics instruction

- Transcribed interviews

\section{GRADUATE RESEARCH ASSISTANT}

University of Louisville

Supervised by Caroline Sheffield, Ph.D.

- Transcribed interviews

- Reviewed research literature regarding social studies and history instruction

SOCIAL IDENTITY LABORATORY

University of Missouri-Columbia

Directed research under the supervision of Ann Bettencourt, Ph.D.

- Reviewed the literature on the research topic (i.e., cross-sex friendships)

- Developed a hypothesis and measures for the variables under study

- Collected and analyzed data

- Presented results at the Midwestern Psychological Association Conference, April 2009

INFANT COGNITION LABORATORY

University of Missouri-Columbia

Research Assistant supervised by Yuyan Luo, Ph.D.

- Responsible for data collection regarding infant prenatal knowledge of physical aspects of the world.

FELLOWSHIP THROUGH THE ARTS AND SCIENCE University of Missouri-Columbia Undergraduate ReSEARCH MeNTOR Program

Supervised by Moshe Naveh-Benjamin, Ph.D.

- Conducted a research study assessing the effects of education on associative memory of older adults.

- Presented the results and submitted the manuscript to the Arts and Science Undergraduate Research Mentor Program Committee.

MeMory AND COGNITIVE AGING LABORATORY University of Missouri-Columbia Research Assistant, supervised by Moshe Naveh-Benjamin, Ph.D.

- Ran experiments assessing memory of older subjects

- Responsible for data entry and analysis using Microsoft Excel and SPSS 
- Created simple programs and configurations for experiments

- Recruited subjects for future experiments and coordinated subject pool

\section{ASSESSMENT EXPERIENCE}

July 2013 -

Aug 2013

Aug 2009 -

May 2011
AdVANCED ASSESSMENT PRACTICUM

Supervised by Jeff Hicks, Ph.D.

- Administered Stanford-Binet, $5^{\text {th }}$ edition, Vineland-II and Kaufman Brief Intelligence Test (K-Bit) to developmentally and intellectually delayed adults at the residential facility.

- Scored Stanford-Binet, $5^{\text {th }}$ edition, Vineland-II and K-Bit and wrote integrative reports regarding the results of the assessments and recommendations for future mental health care

\section{A BetTer Chance CliniC}

University of Oklahoma

\section{HEALTH SCIENCES CENTER}

Graduate Research Assistant supervised by Lisa Swisher, Ph.D.

- Administered Bayley Scales of Infant Development, Third Edition (Bayley-III), the Preschool Language Scale, Fourth Edition (PLS-IV) and the Wechsler Preschool and Primary Scale of Intelligence, Third Edition (WPPSI-III) to children ages 0-7 years, who have been prenatally exposed to drugs and alcohol

- Scored Bayley-III, WPPSI-III and PLS-4 and wrote integrative reports regarding the results of the assessments

- Scored and interpreted the Eyeberg Child Behavior Inventory (ECBI), the Child Behavior Checklist (CBCL), and the Parent Stress Index (PSI) to include in the integrative reports.

- Conducted intakes and delivered feedback and recommendations to parents regarding the development of their children

- Acquired a total of 165 direct assessment hours.

- Trained in Parent-Child Interaction Therapy (PCIT), supervised by Melanie Nelson, $\mathrm{Ph} . \mathrm{D}$. and provided counseling services to families. Acquired a total of 37 direct therapy intervention hours.

\section{OUTREACH}

Jan 2016

Dec 2015

Oct 2015

Aug 2015

Oct 2013
Resident Assistant Training Part II

Suffolk University

- Designed and delivered part of the training program for Resident Assistants. Training included review of handling most common mental health issues and role plays.

OUTREACH PRESENTATION ON CULTURE SHOCK

Suffolk University

- Designed and delivered a psychoeducational lecture on culture shock for students embarking on their study abroad experience

NATIONAL DEPRESSION SCREENING DAY

Suffolk University

- Provided mental health screenings and distributed mental health resources to the student population

Resident Assistant Training Part I

Suffolk University

- Designed and delivered part of the training program for Resident Assistants on working with students who struggle with anxiety

OUTREACH PRESENTATION FOR STUDY ABROAD STUDENTS

Bellarmine University 
- Delivered a psychoeducational lecture on study abroad experience and sexual assault awareness

Oct 2013

CHOOSING GRADUATE PROGRAM OUTREACH

Bellarmine University

- Spoke to a classroom of undergraduate students majoring in psychology about the process of choosing a graduate school program and my experiences in graduate school

Oct 2013

Mental Health AWARENESS Day SCREENINGS

University of Louisville

- Provided mental health screenings and distributed mental health resources to the student population

Sep 2013

OUTREACH PRESENTATION ON STRESS MANAGEMENT

Bellarmine University

- Delivered a psychoeducational lecture on stress management to nursing students

Sep 2013

INTRODUCTION TO COUNSELING OUTREACH

Bellarmine University

- Delivered several psychoeducational lectures on introduction to counseling services to freshmen students

\section{PROFESSIONAL DEVELOPMENT}

Jan 2016 -

May 2016

Sep 2014 -

Oct 2014

March 2014

March 2014

Nov 2013

Oct 2013

Nov 2012

Sep 2012
DBT TRAINING

Alayna L. Harned, LMHC

- 12-week workshop on Dialectical Behavior Therapy (DBT) theory and core treatment strategies, skills and techniques

MEDITATION WORKSHOP

Peter Buecker, M.D.

- Four-week workshop on meditation practice

COUNSELING PSyCHOLOGY CONFERENCE

Atlanta, GA

EATING DISORDER WORKSHOP

Katie Bradley, Psy.D.

- Workshop for engaging and assessing the client with eating disorder symptomology

SENSORIMOTOR PSYCHOTHERAPY TRAINING

Katie Dine Young, Psy.D.

- Training for conducting sensorimotor psychotherapy to work with trauma survivors

COUPLES THERAPY WORKSHOP

Jesse Owen, Ph.D., Johanna Strokoff, M.A.

University of Louisville

- Training for conducting couple's therapy

Trans 101 TRAINING

Stephanie Budge, Ph.D.

- Training directed at raising awareness about transgender culture, terminology, and unique issues that this population faces

AgES \& STAGes QuestionNaIRE (ASQ) TRAINING Seven Counties Services, Inc.

- Training for administering, scoring and interpreting the Ages \& Stages Questionnaire 
Christabel Suthers, M.Ed., Collyn Moone, M.S.W.

- Training for effectively handling crisis situations in the school system and helping children and their families work through trauma

April 2010

BRIEF THERAPY TRAINING

University of Oklahoma

Denise Beesley, Ph.D., Brent Horner, M.Ed., Shelby Ross, M.Ed., Joaquin LawsRodriguez, M.Ed.

- Training for conducting Brief Therapy for the OU Counseling Clinic pro bono program

March 2010

AUDITED PCIT TRAINING

University of Oklahoma Health Sciences Center Beverly Funderback, Ph.D., Melanie Nelson, Ph.D.

- Training for conducting Parent-Child Interaction Therapy (PCIT)

Dec 2009

MARRIAGE \& COUPLES THERAPY WORKSHOP

University of Oklahoma Cal Stoltenberg, Ph.D.

- Training for conducting Integrative Behavioral Couples Therapy

April 2009

MidWESTERN PSYCHOLOGICAL ASSOCIATION CONFERENCE

Chicago, IL

\section{SKILLS AND ABILITIES}

- Working knowledge of administration, scoring and interpretation of CCAPS-34, MMPI2, TAT, MCMI-II, Bayley-III, PLS-4, WPPSI-III, WAIS-IV, WISC-IV, Stanford-Binet, $5^{\text {th }}$ edition, Vineland-II and K-BIT.

- IRB Human Subjects Certified

- HIPPA trained

- Fluent in Russian language

- Diverse cultural experiences and knowledge: lived in three different countries - Japan (4 years), Germany (2 years) and Russia (7 years); traveled in Europe, North and South America, and Australia 